\title{
(9-Isocyanoanthracene)gold(I) Complexes Exhibiting Two Modes of Crystal Jumps by Different Structure Change Mechanisms
}

Kenta Kato, ${ }^{\mathrm{a}, \mathrm{b}}$ Tomohiro Seki, ${ }^{\mathrm{a}, \mathrm{c}, *}$ and Hajime $\mathrm{Ito}^{\mathrm{a}, \mathrm{b}, *}$

\footnotetext{
a Division of Applied Chemistry \& Frontier Chemistry Center, Faculty of Engineering, Hokkaido University, Sapporo, Hokkaido 060-8628, Japan.

${ }^{\mathrm{b}}$ Institute for Chemical Reaction Design and Discovery (WPI-ICReDD), Hokkaido University 001-0021, Japan.

${ }^{\mathrm{c}}$ Department of Chemistry, Faculty of Science, Shizuoka University, 836 Ohya, Suruga-ku, Shizuoka 422-8529, Japan.
}

Email: seki.tomohiro@shizuoka.ac.jp; hajito@eng.hokudai.ac.jp

\section{Contents}

1. General

2. Overview of salient active molecular structures

3. Synthesis

4. Preparation of the single crystals of $1,2,3$, and $1_{\mathrm{PD}}$

5. Comparison of the single crystal structures

6. Images of salient effects of 1,2 , and 3

7. Crystallographic parameters of 1 depend on the various temperatures and $1_{\mathrm{PD}}$

8. Single-crystal XRD structure analyses of 1 and $1_{P D}$

9. Photodimerization of 1 upon photoirradiation

10. Comparison of single-crystal structures of 1 and $1_{P D}$

11. Relationship between the crystallographic parameters of 1 and temperatures

12. DSC trace of 1

13. Photodimerization and DSC traces of 2 and 3

14. Single-crystal XRD structure analyses of 3 


\section{General}

All commercially available reagents and solvents are of reagent grade and were used without further purification. Chloro(tetrahydrothiophene)gold(I) complex (S1) was prepared according to the reported procedure. ${ }^{\mathrm{S} 1}$ Solvents for the synthesis were purchased from commercial suppliers, dried over molecular sieves ( $4 \AA$ ). NMR spectra were recorded on a JEOL JNM-ECS400 FT spectrometer using $\mathrm{CDCl}_{3}$ as a solvent and tetramethylsilane as an internal standard. Chemical shifts for ${ }^{1} \mathrm{H}$ NMR are expressed in parts per million ( $\mathrm{ppm})$ relative to internal tetramethylsilane $(\delta=0.00 \mathrm{ppm})$. Chemical shifts for ${ }^{13} \mathrm{C}$ NMR are expressed in ppm relative to internal tetramethylsilane ( $\left.\delta 0.00 \mathrm{ppm}\right)$. Data are reported as follows: chemical shift, number of atoms, multiplicity $(\mathrm{s}=$ singlet, $\mathrm{d}=\mathrm{doublet}$, $\mathrm{dd}=\mathrm{doublet}$ of doublets, $\mathrm{t}=$ triplet, $\mathrm{m}=$ multiplet $)$, coupling constant $(\mathrm{Hz})$, number of protons, and type of atoms. High-resolution mass spectra were recorded on a JEOL JMS-T100LP using electrospray ionization at the Global Facility Center at Hokkaido University. Photographs were obtained using OLYMPUS BX51 or SZX7 microscopes with Olympus DP72, SONY HDR-CX700V. Differential Scanning Calorimetry (DSC) profiles were measured on Hitachi DSC7020 calorimeter. For UV and visible light irradiation on the photosalient effect of $\mathbf{1 , 2}$, and $\mathbf{3}$, an Olympus BX51 fluorescence microscope equipped with an Ushio $100 \mathrm{~W}$ ultrahigh-pressure mercury lamp USH-1030L and Olympus fluorescence mirror unit UMWU2 $\left(\lambda_{\max }=365 \mathrm{~nm}, \lambda=350-370 \mathrm{~nm}, 105 \mathrm{~mW} \mathrm{~cm}^{-2}\right)$, U-MNV2 $\left(\lambda_{\max }=405 \mathrm{~nm}, \lambda=400-410 \mathrm{~nm}\right.$, $\left.226 \mathrm{~mW} \mathrm{~cm}^{-2}\right)$, and U-MWBV2 $\left(\lambda_{\max }=435 \mathrm{~nm}, \lambda=420-440 \mathrm{~nm}, 158 \mathrm{~mW} \mathrm{~cm}^{-2}\right)$ with Olympus U25ND25; BX2 neutral density filter in slider were used. The raw data files were plotted on Igor Pro Version 8.04 .

X-ray diffraction analyses: A suitable crystal was mounted with Paratone oil on a MiTeGen MicroMounts and transferred to the Four-circle Kappa Geometry Goniometer of a RIGAKU XtaLAB Synergy-DW system with $1.2 \mathrm{~kW}$ PhotonJet-DW microfocus rotating anode using graphite monochromated $\mathrm{Cu}-\mathrm{K}_{\alpha}$ radiation and HyPix-6000HE detector. Cell parameters were determined and refined, and raw frame data were integrated using CrysAlis ${ }^{\text {Pro }}$ (Agilent Technologies, 2010). The structures were solved by direct methods with (SHELXT) ${ }^{\mathrm{S} 2}$ and refined by full-matrix least-squares techniques against $F^{2}$ (SHELXL-2018/3) ${ }^{\mathrm{S} 3}$ by using Olex2 software package. ${ }^{\mathrm{S} 4}$ The intensities were corrected for Lorentz and polarization effects. The non-hydrogen atoms were refined anisotropically. Hydrogen atoms were placed using AFIX instructions. Simulated powder patterns were generated with Mercury $4.3^{\mathrm{S} 5}$ from the structures determined by single-crystal diffraction analyses. 


\section{Overview of salient active molecular structures}

Scheme S1. Chemical structures that enable photosalient effects reported previously. ${ }^{\text {S6-27 }}$

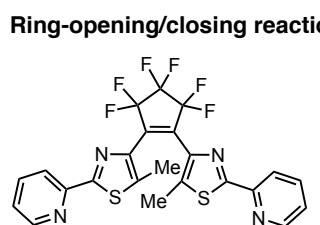

P1. Baldeck, Yu et al. 2005 Baldeck, Yu, Irie et al. 2007.

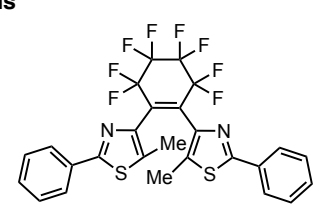

P2. Morimoto, Uchida et al. 2016, Morimoto, Uchida et al. 2017.

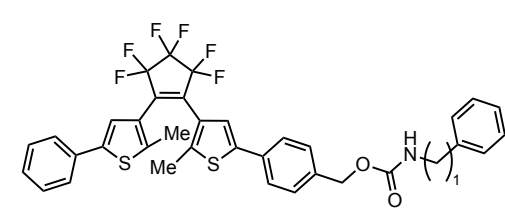

P3 $(n=0), \mathbf{P 4}(n=1)$ Kobatake, et al. 2016.

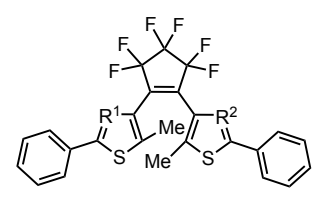

$P 5\left(R^{1}, R^{2}=N, N\right), P 6\left(R^{1}, R^{2}=\right.$ $\mathrm{C}, \mathrm{C}), \mathbf{P} 7\left(\mathrm{R}^{1}, \mathrm{R}^{2}=\mathrm{C}, \mathrm{N}\right)$,

Morimoto, Uchida et al. 2019.

\section{Cycloadditions}<smiles>COc1cccc(/C=C2\N=C(C)N(C)C2=O)c1</smiles>

P8. Naumov, Tolbert et al. 2010.

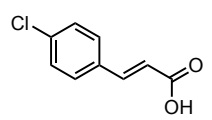

P9. Bardeen et al. 2012

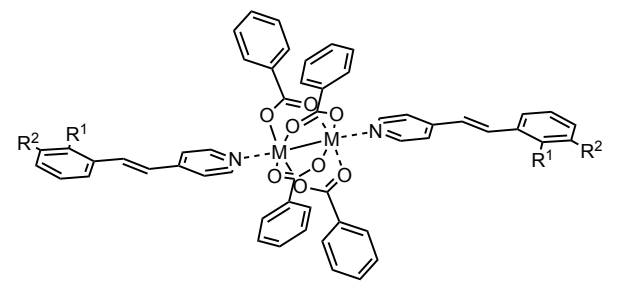

P10 (M, R $\left.{ }^{1}, R^{2}=Z n, H, F\right)$, P11 (Zn,F,H). Naumov, Vittal et al. 2014, P12 $(\mathrm{Zn}, \mathrm{H}, \mathrm{H})$, Vittal et al. 2019

P13 (Cu,H,F), P14 (Cu,F,H), P15 (Cu,H,H), Dinnebier, Vittal et al. 2020

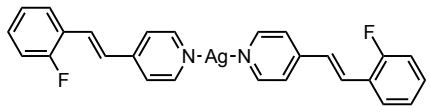

P16. Naumov, Vittal et al. 2015.<smiles>O=C1OCC/C1=C\c1ccccc1</smiles>

P17. Nath et al. 2015.<smiles>COc1ccc(/C=C/C(=O)O)cc1OC</smiles>

P18. Ramamurty, Desirajua et al. 2015.

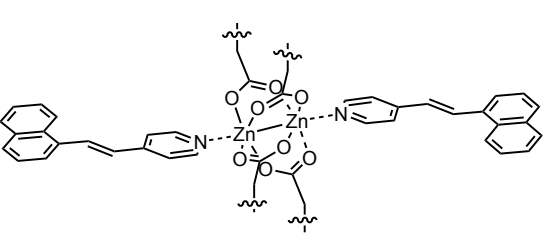

P22. Mir et al. 2019<smiles></smiles>

P19. Vittal et al. 2017.

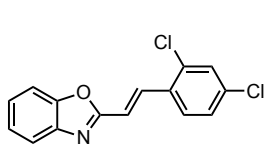

P20. Lu et al. 2017.

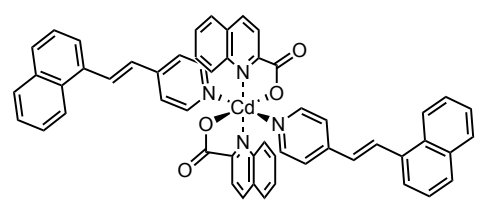

P21. Mir et al. 2019.

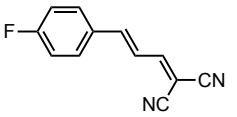

P23. Al-Kaysi, Bardeen et al. 2020

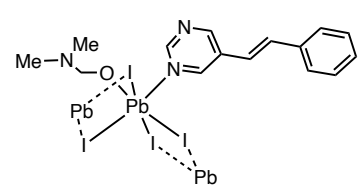

P24. Rath and Vittal 2020.
Rearrangement

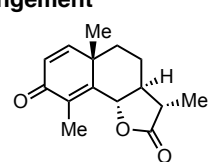

P25. Matsuura et al. 1968 , Naumov, Garcia-Garibay et al. 2015
Linkage isomerization

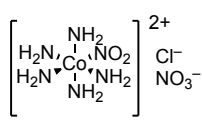

P26. Naumov, Boldyreva et al. 2013 Muya, Chung et al. 2019.
Aurophilicity enhancement

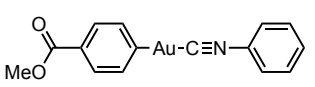

P27. Seki, Ito et al. 2015.
$E \rightarrow Z$ Isomerization<smiles>[R]c1ccc(/C=N/NC(=O)c2ccc([R])cc2)cc1</smiles>

P28 $\left(\mathrm{R}^{1}, \mathrm{R}^{2}=\mathrm{Cl}, \mathrm{OCH}_{3}\right)$,

P29 $\left(R^{1}, R^{2}=F, C N\right)$,

Naumov, Nath et al. 2019. 
Scheme S2. Chemical structures that enable thermosalient effects reported previously. ${ }^{\text {S28-68 }}$ With thermal phase transition

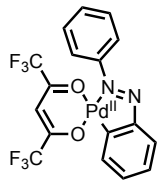

T1. Etter and Siedle 1983 Etter and Siedle 1983, Runčevski, Naumov et al. 2014, Naumov et al. 2019.<smiles>Brc1cc(Br)c(Br)cc1Br</smiles>

T7. Davey et al. 2000 Reddy, Naumov et al. 2013 Naumov et al. 2017 Naumov et al. 2018 Zakharov et al. 2018 Korter et al. 2019.

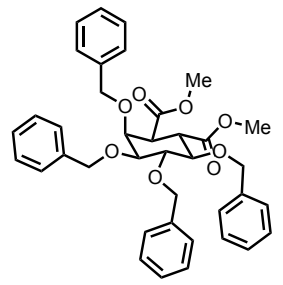

T2. Gigg et al. 1987, Saenger et al. 1993.

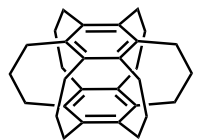

T8. Shinmyozu et al. 2000

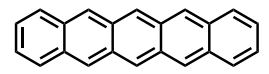

T9. Siegrist et al. 2007.

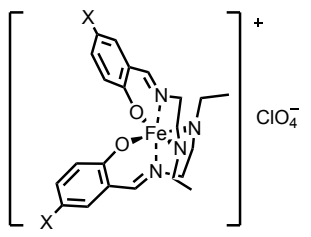

T18 $(X=B r)$. Martinho et al. 2016 T19 $(X=1)$. Martinho et al. 2018.

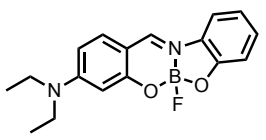

T26. Tanaka, Chujo et al. 2017.

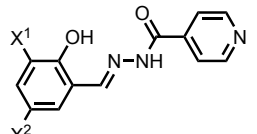

$$
x^{2}
$$

T27 $\left(X^{1}, X^{2}=C l, C l\right)$, T28 $\left(X^{1}, X^{2}=B r, C l\right)$, T29 $\left(X^{1}, X^{2}=B r, B r\right)$, Nangia et al. 2017.

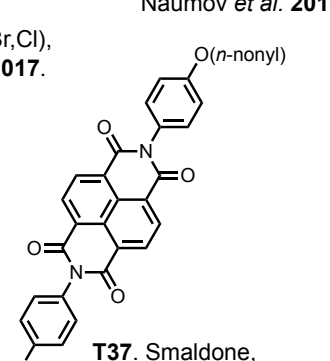

Gassensmith et al. 2018.<smiles>O=C(Oc1cc2ccccc2cc1OC(=O)c1ccc(F)cc1)c1ccc(F)cc1</smiles>

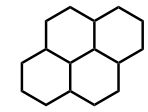

T3. Kohne et al. 1988 Saenger et al. 1991.

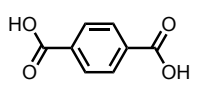

T4. Davey et al. 1994 Naumov et al. 2016.

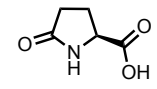

T10. West et al. 2010

Skoko et al. 2017.

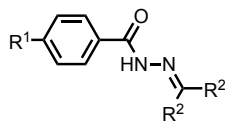

T11 $\left(R^{1}, R^{2}=\mathrm{OH}, \mathrm{CH}_{3}\right)$. Centore et al. 2012

$\mathrm{T} 12\left(\mathrm{R}^{1}, \mathrm{R}^{2}=\mathrm{OD}, \mathrm{CH}_{3}\right), \mathrm{T} 13\left(\mathrm{R}^{1}, \mathrm{R}^{2}=\mathrm{OH}, \mathrm{CD}_{3}\right)$.

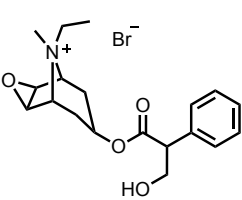

T5. Greenwood et al. 1994

Naumov, Bernstein et al. 2010.

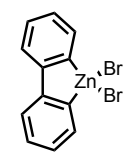

T14. Lusi and Bernstein 2013.

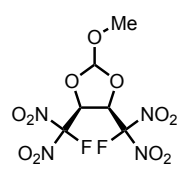

T6. Dickman et al. 1996

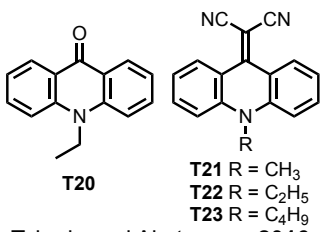<smiles>NC(=O)c1ccc(/N=C/c2cc(Cl)cc(Cl)c2O)cc1</smiles>

T24. Nangia et al. 2017 Vittal et al. 2017.

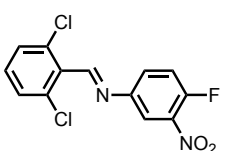

T16. Desiraju et al. 2015

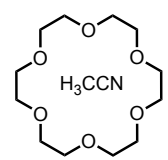

T15. Lusi et al. 2016. T17. Barbour et al. 2016.
Runčevski, Naumov et al. 2015

Centore, Naumov et al. 2016.

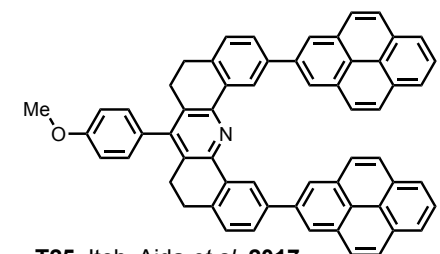

T25. Itoh, Aida et al. 2017.
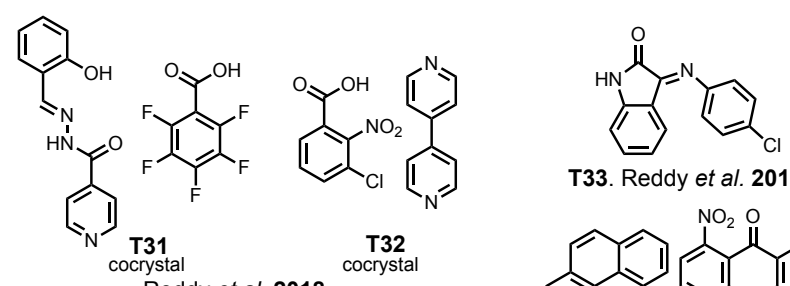

T33. Reddy et al. 2018

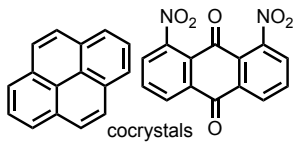

T34 Singh and Chopra 2018.<smiles>CCCN1C(=O)C2=C(c3ccc(Cl)cc3)N(CCC)C(=O)C2=C1c1ccc(Cl)cc1</smiles>

T38. Matsumoto et al. 2018. T39. Garcia-Garibay, Naumov, Rodríguez-Molin et al. 2019.

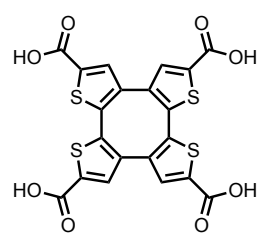

T40. Takeda et al. 2019.

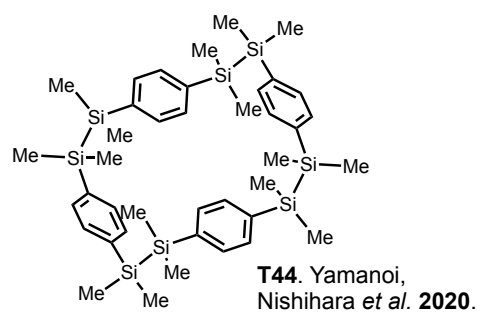

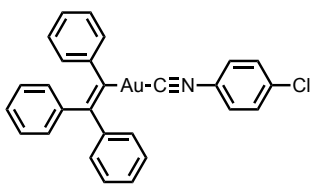

T41. Seki, lto et al. 2019.

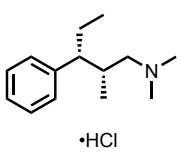
et al. 2019.
T42. Gaztañaga<smiles>C1=C2c3cccc4cccc(c34)C2c2cccc3cccc-3c21</smiles>

T43. Zhang, Zhang, Huang et al. 2020.

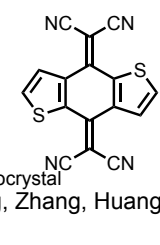

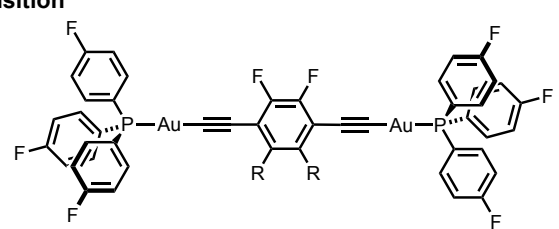

T47 (R = D), T48 (R = F). Ito, Garcia-Garibay et al. 2019

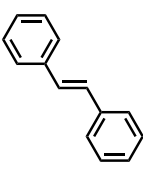

T49

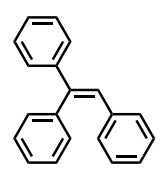

T50 Seki, Ito et al. 2020

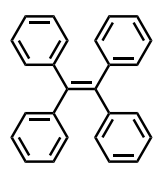

T51

T46. Skoko et al. 2018. 


\section{Synthesis}

Synthesis of 9-isocyanoanthracene

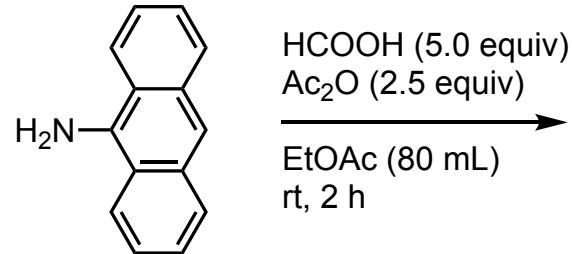

9-aminoanthracene $(1.52 \mathrm{~g}, 8.00 \mathrm{mmol})$

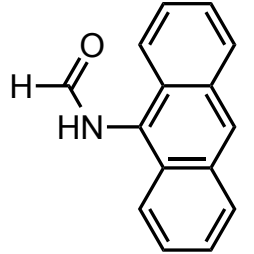

$$
\begin{aligned}
& \mathrm{POCl}_{3}(1.5 \text { equiv }) \\
& \mathrm{Et}_{3} \mathrm{~N}(4.0 \text { equiv }) \\
& \underset{\mathrm{CH}_{2} \mathrm{Cl}_{2}(14 \mathrm{~mL})}{\longrightarrow} \\
& 0{ }^{\circ} \mathrm{C}, 30 \mathrm{~min}, \\
& \text { then } \mathrm{rt}, 2 \mathrm{~h}
\end{aligned}
$$

$N$-(anthracen-9-yl)formamide

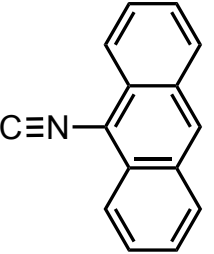

9-isocyanoanthracene (956 mg, $4.7 \mathrm{mmol}, 67 \%$ )

To a dried and nitrogen-purged round-bottom flask containing a magnetic stir bar, formic acid (4.72 $\mathrm{mL}, 40.0 \mathrm{mmol}, 5.0$ equiv) and acetic anhydride $(5.91 \mathrm{~mL}, 20.0 \mathrm{mmol}, 2.5$ equiv) were added. The mixture was stirred at $\mathrm{rt}$ for $1 \mathrm{~h}$ for in-situ preparation of acetic formic anhydride. A solution of 9aminoanthracene $(1.52 \mathrm{~g}, 8.00 \mathrm{mmol})$ in ethyl acetate (EtOAc, $80 \mathrm{~mL}$ ) was prepared in another flask, and the preformed acetic formic anhydride solution was injected to the 9-aminoanthracene solution at $0{ }^{\circ} \mathrm{C}$. After stirring at $\mathrm{rt}$ for $2 \mathrm{~h}$, the removal of volatiles under reduced pressure gave $N$-(anthracen-9yl)formamide as a pale-yellow solid. This was used for the next step without further purification.

An oven-dried two-neck round-bottom flask was connected to a vacuum/nitrogen manifold through a rubber tube. It was evacuated and then backfilled with nitrogen. This cycle was repeated three times. This flask was charged with $N$-(anthracen-9-yl)formamide prepared by the above procedure. The $\mathrm{CH}_{2} \mathrm{Cl}_{2}(14 \mathrm{~mL})$ and trimethylamine $\left(4.45 \mathrm{~mL}, 32 \mathrm{mmol}, 4.0\right.$ equiv) were added and stirred at $0{ }^{\circ} \mathrm{C}$. $\mathrm{POCl}_{3}\left(1.12 \mathrm{~mL}, 12 \mathrm{mmol}, 1.5\right.$ equiv) was added dropwise and stirred at $0{ }^{\circ} \mathrm{C}$ for $30 \mathrm{~min}$. After additional stirring for $2 \mathrm{~h}$ at rt, the reaction mixture was diluted with $\mathrm{CH}_{2} \mathrm{Cl}_{2}$, and washed with icecold sat. aq. $\mathrm{Na}_{2} \mathrm{CO}_{3}$ solution. After filtration, the organic layer was dried over anhydrous $\mathrm{Na}_{2} \mathrm{SO}_{4}$, concentrated under reduced pressure, and purified by flash column chromatography (eluent: $\mathrm{CH}_{2} \mathrm{Cl}_{2}$ ) on silica gel to afford 9-isocyanoanthracene as pale-yellow solids ( $956 \mathrm{mg}, 4.70 \mathrm{mmol}, 67 \%$ ).

${ }^{1} \mathrm{H} \mathrm{NMR}\left(\mathrm{CDCl}_{3}, 400 \mathrm{MHz}\right): \delta 8.50(\mathrm{~s}, 1 \mathrm{H}, \mathrm{ArH}), 8.37-8.34(\mathrm{~m}, 2 \mathrm{H}, \mathrm{ArH}), 8.04(\mathrm{~d}, J=8.4,2 \mathrm{H}$, ArH), 7.70-7.66 (m, 2H, ArH), 7.58-7.54 (m, 2H, ArH). $\left.{ }^{13} \mathrm{C} \mathrm{NMR} \mathrm{(CDCl} 3,100 \mathrm{MHz}\right): \delta 171.14(\mathrm{CN})$, $130.91(\mathrm{C}), 128.72(\mathrm{CH}), 128.61(\mathrm{CH}), 128.11(\mathrm{CH}), 126.96(\mathrm{C}), 126.27(\mathrm{CH}), 123.15(\mathrm{CH}), 118.60$ (C); ESI-MS (m/z): [M+H] $]^{+}$calcd. for $\mathrm{C}_{15} \mathrm{H}_{10} \mathrm{~N}, 204.08078$; found, 204.08082. 
Synthesis of chloro(9-isocyanoanthracene)gold(I) (S2)

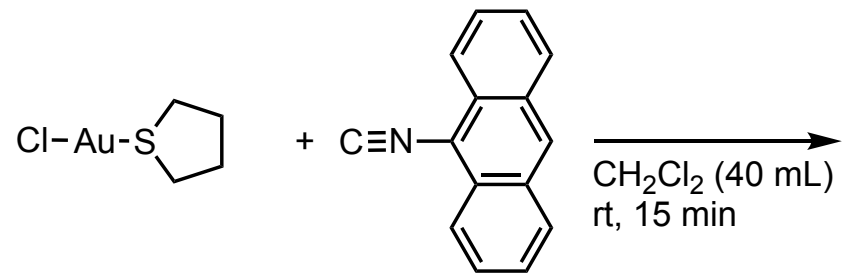

S1

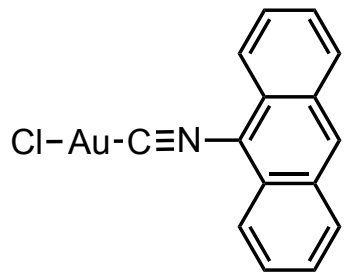

S2

$(1.28 \mathrm{~g}, 4.00 \mathrm{mmol}) \quad$ (1.05 equiv)

(1.83 g, $4.00 \mathrm{mmol}$, quant.)

An oven-dried two-neck round-bottom flask was connected to a vacuum/nitrogen manifold through a rubber tube. It was evacuated and then backfilled with nitrogen. This cycle was repeated three times. In this flask, chloro(tetrahydrothiophene)gold(I) (S1; $1.282 \mathrm{~g}, 4.00 \mathrm{mmol})$ and 9-isocyanoanthracene (853.6 mg, $4.20 \mathrm{mmol}, 1.05$ equiv) were dissolved in $\mathrm{CH}_{2} \mathrm{Cl}_{2}(40 \mathrm{~mL})$ and stirred for $15 \mathrm{~min}$ at ambient temperature. The solvent was removed with a rotary evaporator under reduced pressure. Washing the crude mixture with methanol gave an analytically pure yellow solids of chloro(9-isocyanide anthracene)gold(I) (S2; $1.83 \mathrm{~g}, 4.00 \mathrm{mmol}$, quantitative).

${ }^{1} \mathrm{H} \mathrm{NMR}\left(\mathrm{CDCl}_{3}, 400 \mathrm{MHz}\right): \delta 8.72(\mathrm{~s}, 1 \mathrm{H}, \mathrm{ArH}), 8.20(\mathrm{dd}, J=9.2,1.0,2 \mathrm{H}, \mathrm{ArH}), 8.14(\mathrm{~d}, J=$ 8.8, 2H, ArH), 7.83-7.79 (m, 2H, ArH), 7.68-7.64 (m, $2 \mathrm{H}, \mathrm{ArH})$; ESI-MS (m/z): $[\mathrm{M}+\mathrm{Na}]^{+}$calcd. for $\mathrm{C}_{15} \mathrm{H}_{9} \mathrm{AuClNNa}, 457.99867$; found, 457.99811. This compound exhibits poor solubility in common organic solvents; therefore, ${ }^{13} \mathrm{C}$ NMR spectroscopy can not be measured. 
Synthesis of aryl(9-isocyanoanthracene)gold(I) complexes 1, 2, and $\mathbf{3}$

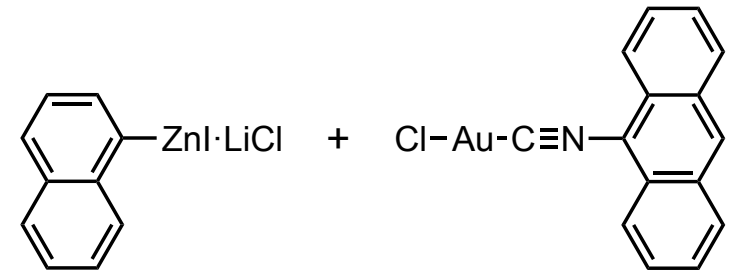

S2

(1.5 equiv)

$(450 \mu \mathrm{mol})$

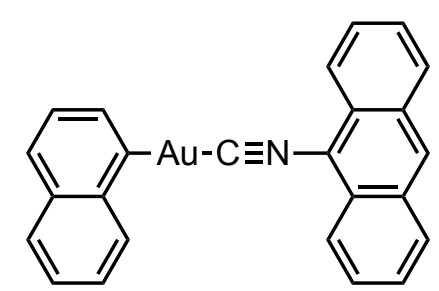

1

(219 mg, $415 \mu \mathrm{mol}, 92 \%)$

An oven-dried two-neck round-bottom flask was connected to a vacuum/nitrogen manifold through a rubber tube. It was evacuated and then backfilled with nitrogen. This cycle was repeated three times. In this flask, S2 $(196 \mathrm{mg}, 450 \mu \mathrm{mol})$ was suspended in THF $(45 \mathrm{~mL})$. After cooling to $0{ }^{\circ} \mathrm{C}$, a THF solution of 1-naphthylzinc iodide-lithium chloride $(240 \mathrm{mM}, 2.81 \mathrm{~mL}, 675 \mu \mathrm{mol}, 1.5$ equiv) was added dropwise with stirring. After $15 \mathrm{~min}$, the reaction was quenched by the addition of a phosphate buffer solution and then extracted with $\mathrm{CH}_{2} \mathrm{Cl}_{2}$ three times and washed with $\mathrm{H}_{2} \mathrm{O}$ and brine. The organic layers were collected and dried over $\mathrm{Na}_{2} \mathrm{SO}_{4}$. After filtration, the solvent was removed in vacuo. The crude solid was dissolved in $\mathrm{CH}_{2} \mathrm{Cl}_{2}$ and reprecipitated by hexane addition to give analytically pure 1-naphthyl(9-isocyanoanthracene)gold(I) (1) as a yellow solid (219 mg, $415 \mu \mathrm{mol}, 92 \%)$.

${ }^{1} \mathrm{H} \mathrm{NMR}\left(\mathrm{CDCl}_{3}, 400 \mathrm{MHz}\right): \delta 8.71(\mathrm{~s}, 1 \mathrm{H}, \mathrm{ArH}), 8.61(\mathrm{~d}, J=8.0 \mathrm{~Hz}, 1 \mathrm{H}, \mathrm{ArH}), 8.36(\mathrm{~d}, J=8.8$ $\mathrm{Hz}, 2 \mathrm{H}, \mathrm{ArH}), 8.15$ (d, J=8.4 Hz, 2H, ArH), 7.84-7.80 (m, 3H, ArH), 7.71-7.66 (m, 4H, ArH), 7.497.42 (m, 3H, ArH); ESI-MS (m/z): [M+Na $]^{+}$calcd. for $\mathrm{C}_{25} \mathrm{H}_{16} \mathrm{AuNNa}, 550.08459$; found, 550.08453. This compound exhibits poor solubility in common organic solvents; therefore, ${ }^{13} \mathrm{C}$ NMR spectroscopy can not be measured.

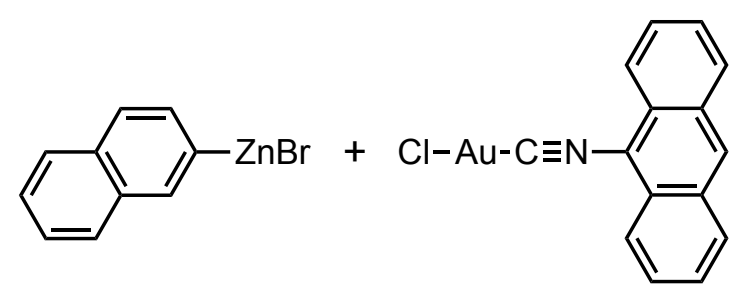

S2

(1.5 equiv)

(700 $\mu \mathrm{mol})$

$\overrightarrow{\mathrm{THF}}(70 \mathrm{~mL})$ $0{ }^{\circ} \mathrm{C}, 15 \mathrm{~min}$

2-Naphthyl(9-isocyanoanthracene)gold(I) (2) was prepared from S2 (305 mg, $700 \mu \mathrm{mol})$ and a THF solution of 2-naphthylzinc bromide $(500 \mathrm{mM}, 2.10 \mathrm{~mL}, 1.05 \mathrm{mmol}, 1.5$ equiv) according to the procedure similar to that described for the preparation of 1. Yield: $364 \mathrm{mg}, 690 \mu \mathrm{mol}, 99 \%$.

${ }^{1} \mathrm{H} \mathrm{NMR}\left(\mathrm{CDCl}_{3}, 400 \mathrm{MHz}\right): \delta 8.70(\mathrm{~s}, 1 \mathrm{H}, \mathrm{ArH}), 8.33$ (d, $\left.J=8.4 \mathrm{~Hz}, 2 \mathrm{H}, \mathrm{ArH}\right), 8.14(\mathrm{~d}, J=8.8$ $\mathrm{Hz}, 2 \mathrm{H}, \mathrm{ArH}), 8.04$ (s, 1H, ArH), 7.82-7.70 (m, 6H, ArH), 7.65 (t, $J=8.4 \mathrm{~Hz}, 2 \mathrm{H}, \mathrm{ArH}), 7.42$ (t, $J=$ $6.8 \mathrm{~Hz}, 1 \mathrm{H}, \mathrm{ArH}), 7.35(\mathrm{t}, J=7.2 \mathrm{~Hz}, 1 \mathrm{H}, \mathrm{ArH})$; ESI-MS $(\mathrm{m} / \mathrm{z})$ : $[\mathrm{M}+\mathrm{Na}]^{+}$calcd. for $\mathrm{C}_{25} \mathrm{H}_{16} \mathrm{AuNNa}$, 550.08459; found, 550.08342. This compound exhibits poor solubility in common organic solvents; therefore, ${ }^{13} \mathrm{C}$ NMR spectroscopy can not be measured. 


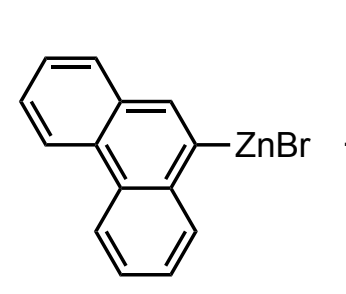

(1.5 equiv)

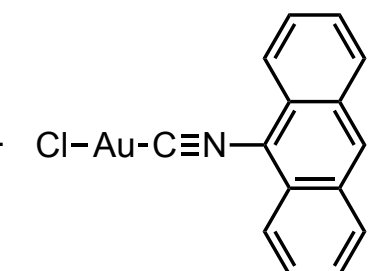

S2

$(700 \mu \mathrm{mol})$

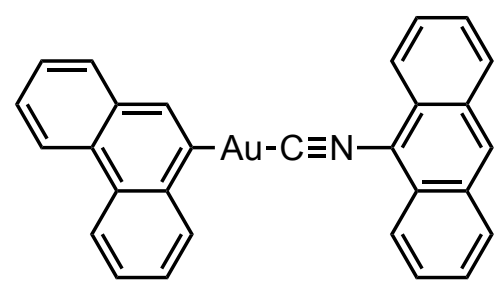

3

(359 mg, $621 \mu \mathrm{mol}, 89 \%)$

9-Phenanthryl(9-isocyanoanthracene)gold(I) (3) was prepared from S2 (305 mg, $700 \mu \mathrm{mol})$ and a THF solution of 9-phenanthrylzinc bromide (500 mM, $2.10 \mathrm{~mL}, 1.05 \mathrm{mmol}, 1.5$ equiv) according to the procedure similar to that described for preparation of 1. Yield: $359 \mathrm{mg}, 621 \mu \mathrm{mol}, 89 \%$.

${ }^{1} \mathrm{H} \mathrm{NMR}\left(\mathrm{CDCl}_{3}, 400 \mathrm{MHz}\right) \delta 8.74-8.72(\mathrm{~m}, 3 \mathrm{H}), 8.68-8.62(\mathrm{~m}, 1 \mathrm{H}), 8.38(\mathrm{~d}, J=8.8,2 \mathrm{H}), 8.16$ $(\mathrm{d}, J=8.4,2 \mathrm{H}), 7.99(\mathrm{~s}, 1 \mathrm{H}), 7.85-7.81(\mathrm{~m}, 3 \mathrm{H}), 7.69-7.52(\mathrm{~m}, 6 \mathrm{H})$; ESI-MS $(\mathrm{m} / \mathrm{z}):[\mathrm{M}+\mathrm{Na}]^{+}$calcd. for $\mathrm{C}_{29} \mathrm{H}_{18} \mathrm{AuNNa}, 600.10024$; found, 600.10017. This compound exhibits poor solubility in common organic solvents; therefore, ${ }^{13} \mathrm{C}$ NMR spectroscopy can not be measured. 


\section{Preparation of the single crystals of $1,2,3$, and 1 PD}

Preparation of crystal samples for salient effects: The single crystals of 1, 2, and $\mathbf{3}$ were obtained by vapor diffusion methods. Typically, $5 \mathrm{mg}$ of $\mathrm{Au}(\mathrm{I})$ isocyanide complex $(\mathbf{1}, \mathbf{2}$, or $\mathbf{3})$ was dissolved in $1.0 \mathrm{~mL}$ of $\mathrm{CHCl}_{3}$. The solution was poured into a small vail. The opened small vial put in a large vial lined with hexane $(3.0 \mathrm{~mL})$ and the large vial was closed by a cap. The crystalline sample was obtained at room temperature in dark for few days.

Preparation of $\mathbf{1}_{\text {PD }}$ crystal samples for X-ray structure analysis: Single crystals of $\mathbf{1}$ were placed in a Paratone oil drop on a glass plate. The glass plate was placed by the window to expose to the sunlight through the window glass. To avoid dust contamination, we covered upside down with a Petri dish and left for a week to give single crystals of $\mathbf{1}_{\mathbf{P D}}$. The sunlight intensity is approx. $1 \mathrm{~mW} \mathrm{~cm}^{-2}(390-480$ $\mathrm{nm}$ ). We could not obtain any suitable crystals for X-ray structure analysis by other UV sources because of lowering the crystal quality and fragmentation. 


\section{Comparison of the single crystal structures}

a)

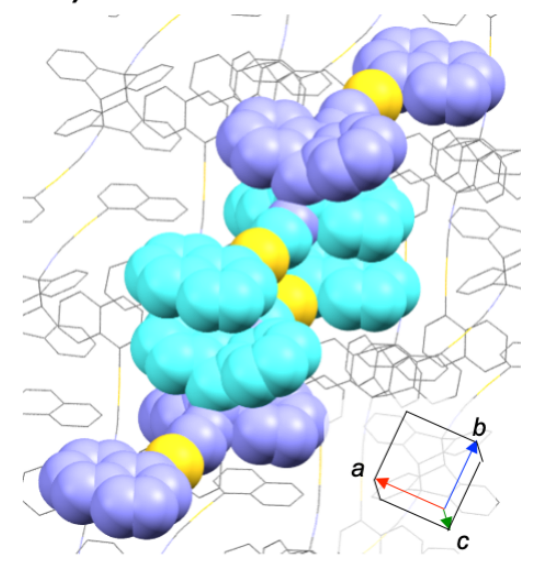

b)

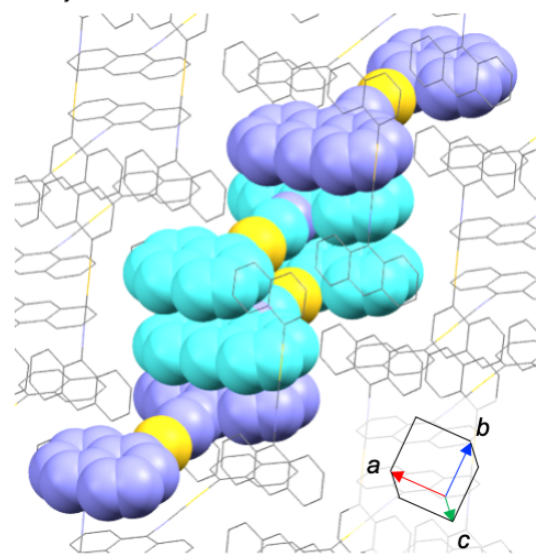

c)

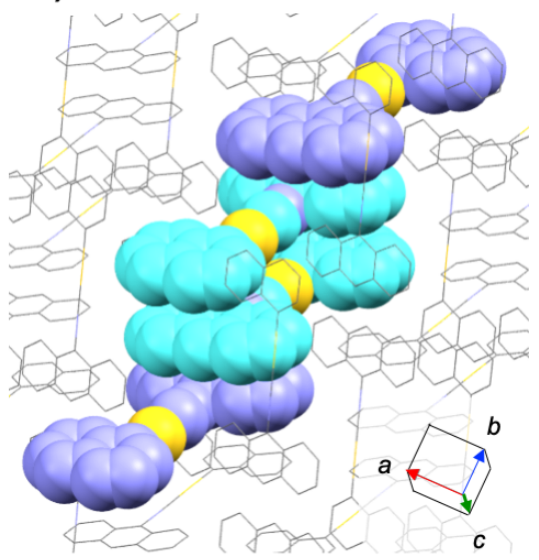

Figure S1. Single crystal structures of a) $\mathbf{1}_{\mathbf{P D}}$, b) 1 at $20^{\circ} \mathrm{C}$, and c) $\mathbf{1}$ at $-140{ }^{\circ} \mathrm{C}$ viewed from the same direction. 


\section{Images of salient effects of 1,2 , and 3}

a) Photoirradiation at $365 \mathrm{~nm}$

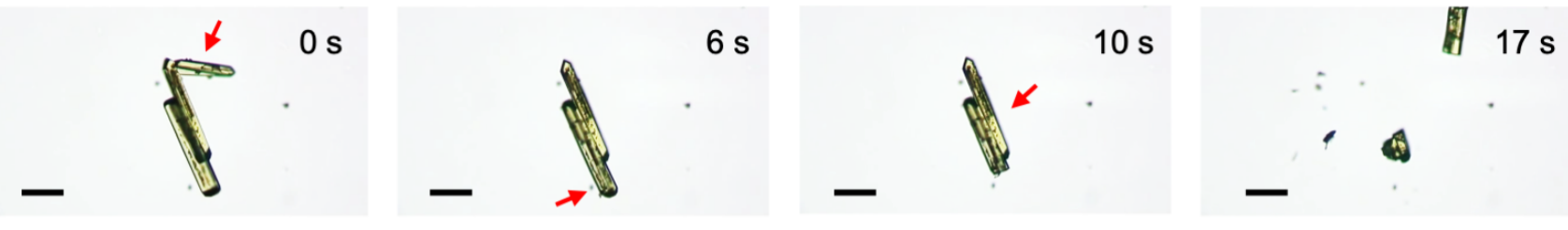

b) Photoirradiation at $405 \mathrm{~nm}$

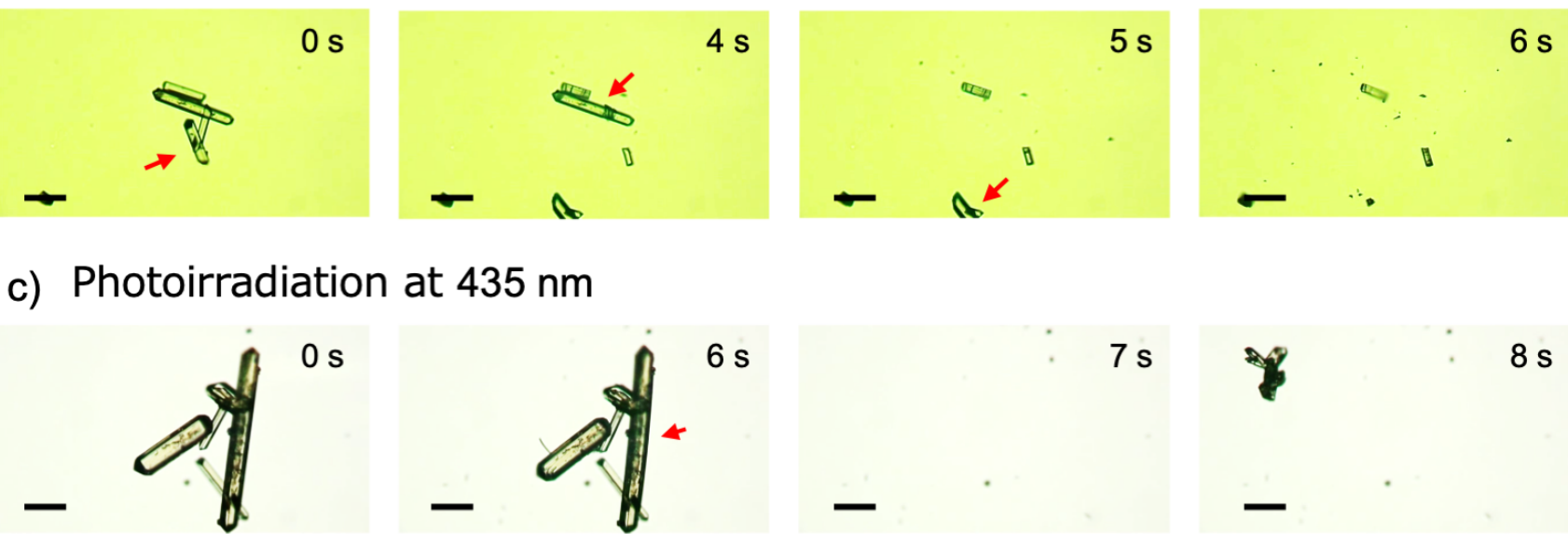

Figure S2. Photographs showing the photosalient effect of 1 initiated by photoirradiation at a) $365 \mathrm{~nm}$, b) $405 \mathrm{~nm}$, and c) $435 \mathrm{~nm}$. Red arrows show the crystal which jump during this observation. Scale bars represent $50 \mu \mathrm{m}$.
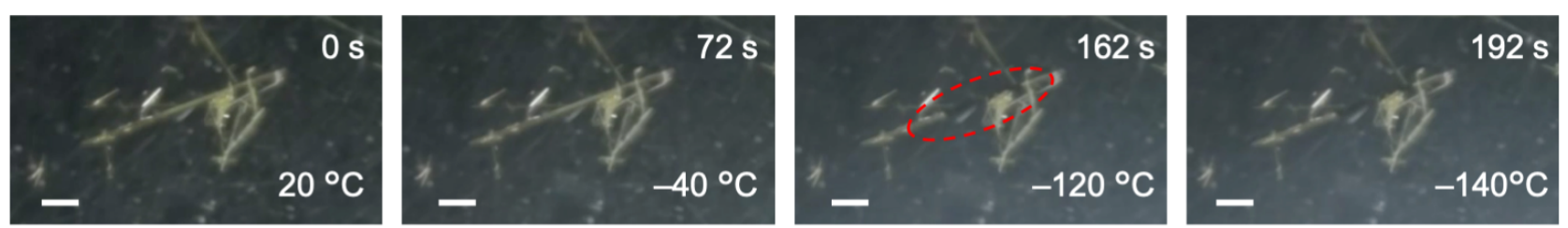

Figure S3. Photographs showing thermosalient effect of 1 upon cooling with at $50{ }^{\circ} \mathrm{C} / \mathrm{min}$. Red ellipse shows the crystal which jump during this observation. Scale bars represent $200 \mu \mathrm{m}$. 
a) Photoirradiation at $365 \mathrm{~nm}$

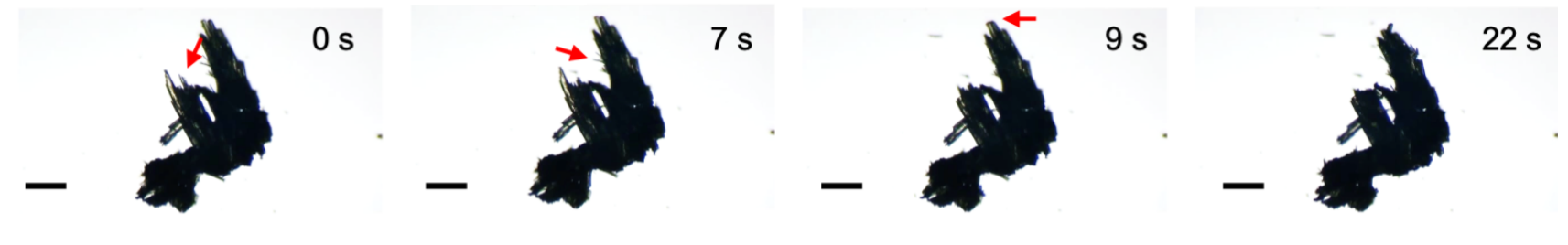

b) Photoirradiation at $405 \mathrm{~nm}$

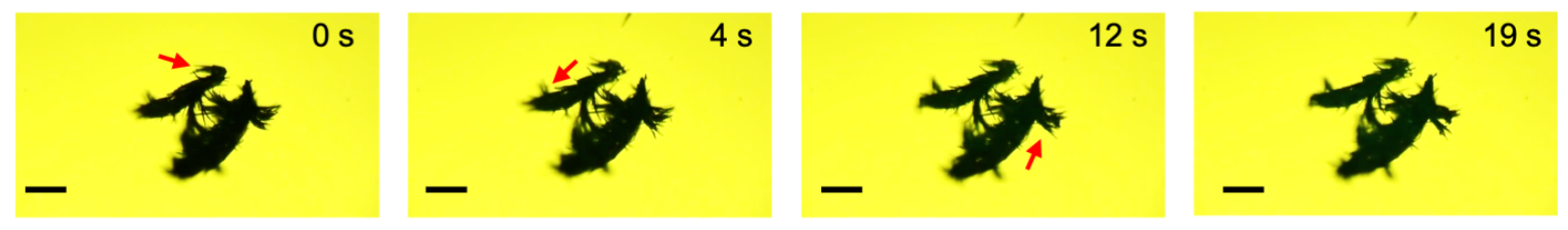

c) Photoirradiation at $435 \mathrm{~nm}$

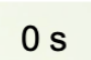

4 $\lambda^{4} 4.5 \mathrm{~s}$

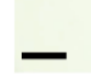

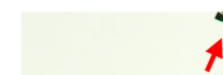

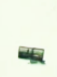

Figure S4. Photographs showing the photosalient effect of 2 initiated by photoirradiation at a) $365 \mathrm{~nm}$, b) $405 \mathrm{~nm}$, and c) $435 \mathrm{~nm}$. Red arrows show the crystal which jump during this observation. Scale bars represent $50 \mu \mathrm{m}$. 
a) Photoirradiation at $365 \mathrm{~nm}$

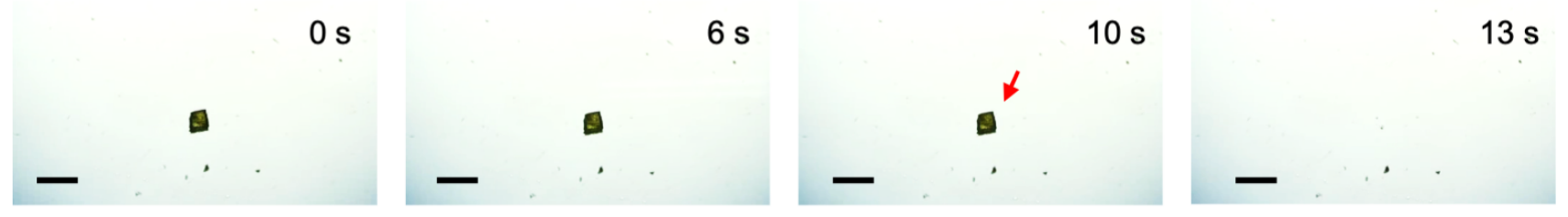

b) Photoirradiation at $405 \mathrm{~nm}$

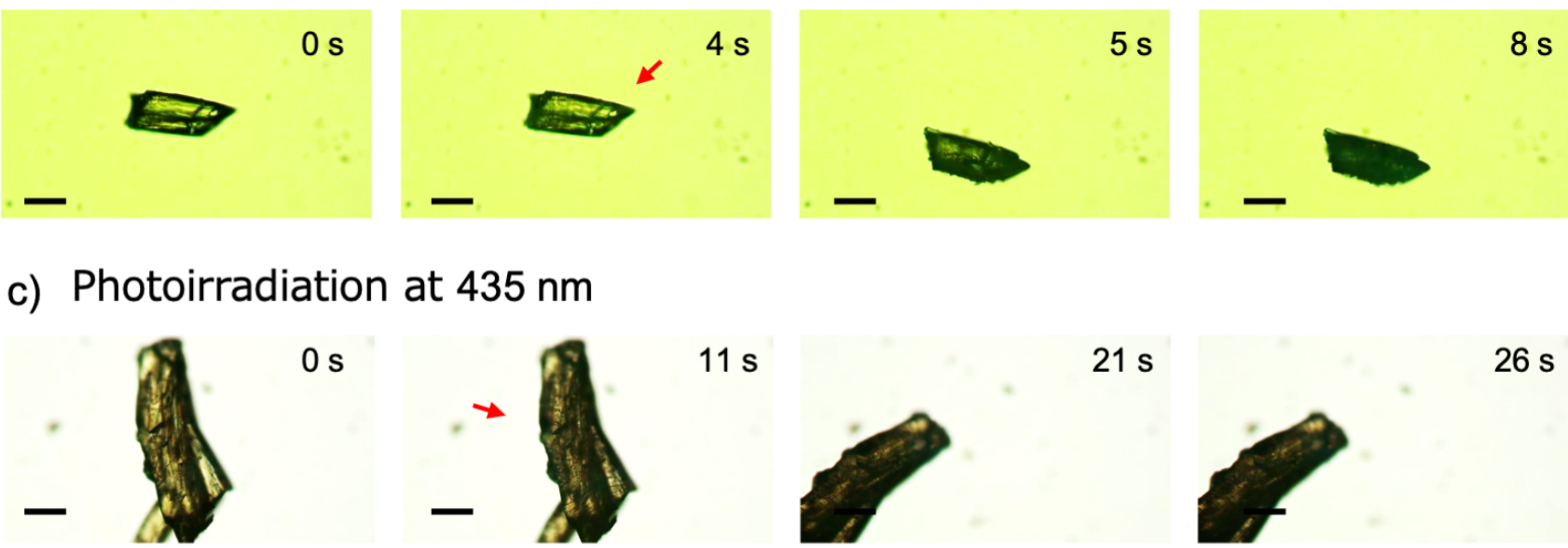

Figure S5. Photographs showing the photosalient effect of 3 initiated by photoirradiation at a) $365 \mathrm{~nm}$, b) $405 \mathrm{~nm}$, and c) $435 \mathrm{~nm}$. Red arrows show the crystal which jump during this observation. Scale bars represent $50 \mu \mathrm{m}$.

a)
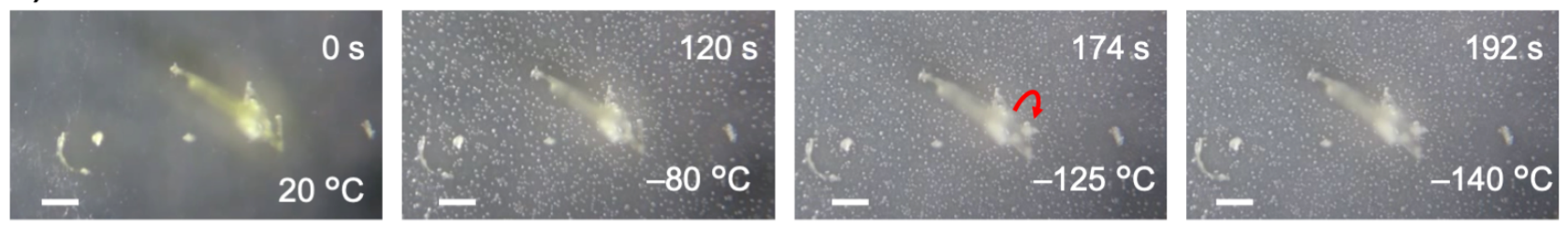

b)
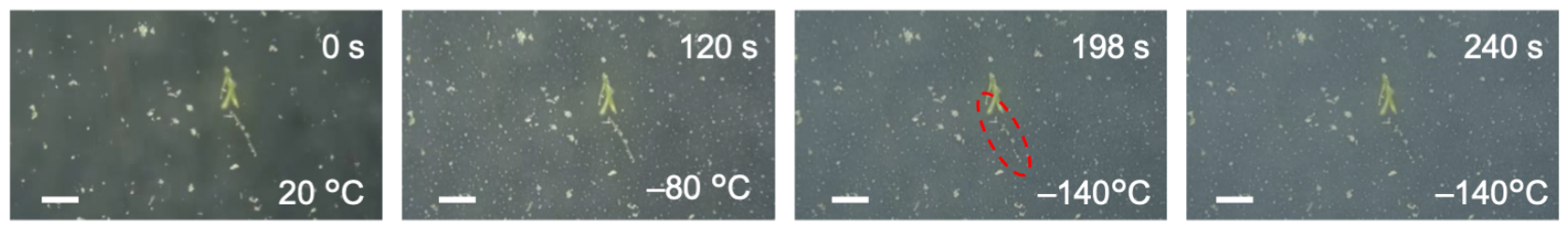

Figure S6. Photographs showing thermosalient effect of a) 2 and b) 3 upon cooling at $50{ }^{\circ} \mathrm{C} / \mathrm{min}$. Red arrow and ellipse show the crystal which jump during this observation. Scale bars represent $200 \mu \mathrm{m}$. 


\section{Crystallographic parameters of 1 depend on the various temperatures and $1_{P D}$}

Table S1. Summary of X-ray crystallographic data for $\mathbf{1}$ at $20^{\circ} \mathrm{C}$ to $-140{ }^{\circ} \mathbf{C}$ and $\mathbf{1}_{\mathbf{P D}}$ at $20^{\circ} \mathbf{C}$.

\begin{tabular}{|c|c|c|c|c|c|}
\hline compound & 1 at $-140^{\circ} \mathrm{C}$ & 1 at $-120^{\circ} \mathrm{C}$ & 1 at $-100{ }^{\circ} \mathrm{C}$ & 1 at $-80^{\circ} \mathrm{C}$ & 1 at $-60^{\circ} \mathrm{C}$ \\
\hline CCDC Number & 2018148 & 2074056 & 2074057 & 2074058 & 2074059 \\
\hline Empirical Formula & $\mathrm{C}_{25} \mathrm{H}_{16} \mathrm{AuN}$ & $\mathrm{C}_{25} \mathrm{H}_{16} \mathrm{AuN}$ & $\mathrm{C}_{25} \mathrm{H}_{16} \mathrm{AuN}$ & $\mathrm{C}_{25} \mathrm{H}_{16} \mathrm{AuN}$ & $\mathrm{C}_{25} \mathrm{H}_{16} \mathrm{AuN}$ \\
\hline Formula Weight & 527.35 & 527.35 & 527.35 & 527.35 & 527.35 \\
\hline Crystal Size / mm & $0.10 \times 0.10 \times 0.10$ & $0.10 \times 0.10 \times 0.10$ & $0.10 \times 0.10 \times 0.10$ & $0.10 \times 0.10 \times 0.10$ & $0.10 \times 0.10 \times 0.10$ \\
\hline Crystal System & monoclinic & monoclinic & monoclinic & monoclinic & monoclinic \\
\hline$a / \AA$ & $11.23610(10)$ & $11.23720(10)$ & $11.24140(10)$ & $11.24480(10)$ & $11.24400(10)$ \\
\hline$b / \AA$ & $12.13100(10)$ & $12.13600(10)$ & $12.14140(10)$ & $12.14790(10)$ & $12.15540(10)$ \\
\hline$c / \AA$ & $13.5071(2)$ & $13.5280(2)$ & $13.5495(2)$ & $13.5771(2)$ & $13.60150(10)$ \\
\hline$\alpha 1^{\circ}$ & 90 & 90 & 90 & 90 & 90 \\
\hline$\beta /^{\circ}$ & $98.6270(10)$ & $98.6350(10)$ & $98.6500(10)$ & $98.6880(10)$ & $98.6920(10)$ \\
\hline$\gamma /^{\circ}$ & 90 & 90 & 90 & 90 & 90 \\
\hline$V / \AA^{3}$ & $1820.26(4)$ & $1823.96(4)$ & $1828.29(4)$ & $1833.36(4)$ & $1837.64(3)$ \\
\hline Space Group & $P 2_{1} / n(\# 14)$ & $P 2_{1} / n(\# 14)$ & $P 2_{1} / n(\# 14)$ & $P 2_{1} / n(\# 14)$ & $P 2_{1} / n(\# 14)$ \\
\hline$Z$ value & 4 & 4 & 4 & 4 & 4 \\
\hline$D_{\text {calc }} / \mathrm{g} \mathrm{cm}^{-3}$ & 1.924 & 1.920 & 1.916 & 1.911 & 1.906 \\
\hline Temperature / K & $133(2)$ & $153(2)$ & $173(2)$ & $193(2)$ & $213(2)$ \\
\hline $2 \theta_{\max } 1^{\circ}$ & 136.5 & 136.436 & 136.436 & 136.408 & 136.402 \\
\hline$\mu / \mathrm{mm}^{-1}$ & $15.235\left(\mathrm{Cu} \mathrm{K}_{\alpha}\right)$ & $15.204\left(\mathrm{Cu} \mathrm{K}_{\alpha}\right)$ & $15.169\left(\mathrm{Cu} \mathrm{K}_{\alpha}\right)$ & $15.127\left(\mathrm{Cu} \mathrm{K}_{\alpha}\right)$ & $15.091\left(\mathrm{Cu} \mathrm{K}_{\alpha}\right)$ \\
\hline No. of Reflections & $\begin{array}{l}\text { Total: } 16191 \\
\text { Unique: } 3336 \\
R_{\text {int }}=0.0359\end{array}$ & $\begin{array}{l}\text { Total: } 16015 \\
\text { Unique: } 3341 \\
R_{\text {int }}=0.0367\end{array}$ & $\begin{array}{l}\text { Total: } 16346 \\
\text { Unique: } 3344 \\
R_{\text {int }}=0.0371\end{array}$ & $\begin{array}{l}\text { Total: } 15995 \\
\text { Unique: } 3352 \\
R_{\text {int }}=0.0371\end{array}$ & $\begin{array}{l}\text { Total: } 15827 \\
\text { Unique: } 3357 \\
R_{\text {int }}=0.0375\end{array}$ \\
\hline$R_{1^{a}}$ & 0.0441 & 0.0442 & 0.0436 & 0.0441 & 0.0435 \\
\hline$w R_{2}{ }^{\mathrm{b}}$ & 0.1088 & 0.1124 & 0.1112 & 0.1148 & 0.1137 \\
\hline $\mathrm{GOF}^{c}$ & 1.073 & 1.069 & 1.075 & 1.065 & 1.064 \\
\hline Max./Mini. Peak $I / \AA^{3}$ & $6.97 \mathrm{e}^{-/-1.91 \mathrm{e}^{-}}$ & $6.72 \mathrm{e}^{-/-1.74 \mathrm{e}^{-}}$ & $6.24 \mathrm{e}^{-/-1.73 \mathrm{e}^{-}}$ & $6.40 \mathrm{e}^{-/-1.40 \mathrm{e}^{-}}$ & $5.82 \mathrm{e}^{-/-1.86 \mathrm{e}^{-}}$ \\
\hline compound & 1 at $-40^{\circ} \mathrm{C}$ & 1 at $-20^{\circ} \mathrm{C}$ & 1 at $0{ }^{\circ} \mathrm{C}$ & 1 at $20^{\circ} \mathrm{C}$ & $\mathbf{1}_{\mathrm{PD}}$ \\
\hline CCDC Number & 2074060 & 2074061 & 2074062 & 2018153 & 2018114 \\
\hline Empirical Formula & $\mathrm{C}_{25} \mathrm{H}_{16} \mathrm{AuN}$ & $\mathrm{C}_{25} \mathrm{H}_{16} \mathrm{AuN}$ & $\mathrm{C}_{25} \mathrm{H}_{16} \mathrm{AuN}$ & $\mathrm{C}_{25} \mathrm{H}_{16} \mathrm{AuN}$ & $\mathrm{C}_{50} \mathrm{H}_{32} \mathrm{Au}_{2} \mathrm{~N}_{2}$ \\
\hline Formula Weight & 527.35 & 527.35 & 527.35 & 527.35 & 1054.71 \\
\hline Crystal Size / mm & $0.10 \times 0.10 \times 0.10$ & $0.10 \times 0.10 \times 0.10$ & $0.10 \times 0.10 \times 0.10$ & $0.10 \times 0.10 \times 0.10$ & $0.10 \times 0.10 \times 0.05$ \\
\hline Crystal System & monoclinic & monoclinic & monoclinic & monoclinic & monoclinic \\
\hline$a / \AA$ & $11.24410(10)$ & $11.24050(10)$ & $11.23740(10)$ & $11.23340(10)$ & $11.5530(17)$ \\
\hline$b / \AA$ & $12.16240(10)$ & $12.17040(10)$ & $12.17850(10)$ & $12.18860(10)$ & $12.6427(11)$ \\
\hline$c / \AA$ & $13.6315(2)$ & $13.6580(2)$ & $13.6868(2)$ & $13.7168(2)$ & $13.0313(14)$ \\
\hline$\alpha 1^{\circ}$ & 90 & 90 & 90 & 90 & 90 \\
\hline$\beta /^{\circ}$ & $98.7060(10)$ & $98.7070(10)$ & $98.7170(10)$ & $98.7120(10)$ & $100.198(13)$ \\
\hline$\gamma /^{\circ}$ & 90 & 90 & 90 & 90 & 90 \\
\hline$V / \AA^{3}$ & $1842.70(4)$ & $1846.90(4)$ & $1851.47(4)$ & $1856.43(4)$ & $1873.3(4)$ \\
\hline Space Group & $P 2_{1} / n(\# 14)$ & $P 2_{1} / n(\# 14)$ & $P 2_{1} / n(\# 14)$ & $P 2{ }_{1} / n(\# 14)$ & $P 2_{1} / n(\# 14)$ \\
\hline$Z$ value & 4 & 4 & 4 & 4 & 2 \\
\hline$D_{\text {calc }} / \mathrm{g} \mathrm{cm}^{-3}$ & 1.901 & 1.897 & 1.892 & 1.887 & 1.87 \\
\hline Temperature / K & $233(2)$ & $253(2)$ & $273(2)$ & $293(2)$ & $293(2)$ \\
\hline $2 \theta_{\max } /^{\circ}$ & 136.498 & 136.474 & 136.482 & 136.496 & 136.5 \\
\hline$\mu / \mathrm{mm}^{-1}$ & $15.050\left(\mathrm{Cu} \mathrm{K}_{\alpha}\right)$ & $\left.15.016(\mathrm{Cu} \mathrm{K})_{\alpha}\right)$ & $\left.14.979(\mathrm{Cu} \mathrm{K})_{\alpha}\right)$ & $14.939\left(\mathrm{Cu} \mathrm{K}_{\alpha}\right)$ & $14.804\left(\mathrm{Cu} \mathrm{K}_{\alpha}\right)$ \\
\hline No. of Reflections & $\begin{array}{l}\text { Total: } 16604 \\
\text { Unique: } 3372 \\
R_{\text {int }}=0.0384\end{array}$ & $\begin{array}{l}\text { Total: } 15798 \\
\text { Unique: } 3380 \\
R_{\text {int }}=0.0360\end{array}$ & $\begin{array}{l}\text { Total: } 15645 \\
\text { Unique: } 3391 \\
R_{\text {int }}=0.0386\end{array}$ & $\begin{array}{l}\text { Total: } 16242 \\
\text { Unique: } 3396 \\
R_{\text {int }}=0.0388\end{array}$ & $\begin{array}{l}\text { Total: } 10742 \\
\text { Unique: } 3429 \\
R_{\text {int }}=0.0733\end{array}$ \\
\hline$R_{1^{\mathrm{a}}}$ & 0.0437 & 0.0436 & 0.0435 & 0.0425 & 0.0663 \\
\hline$w R_{2} \mathrm{~b}$ & 0.1139 & 0.1127 & 0.1147 & 0.1112 & 0.2071 \\
\hline $\mathrm{GOF}^{c}$ & 1.068 & 1.054 & 1.054 & 1.050 & 1.070 \\
\hline Max./Mini. Peak $I / \AA^{3}$ & 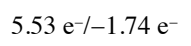 & $5.48 \mathrm{e}^{-/-1.45 \mathrm{e}^{-}}$ & $4.94 \mathrm{e}^{-/-1.42 \mathrm{e}^{-}}$ & $4.63 \mathrm{e}^{-/-1.45 \mathrm{e}^{-}}$ & $1.78 \mathrm{e}^{-/-1.43 \mathrm{e}^{-}}$ \\
\hline
\end{tabular}

[a] For data with $I>2.00 \sigma(I)$. [b] For all reflection data. [c] Goodness of Fit. 


\section{Single-crystal XRD structure analyses of 1 and 1PD}

a)

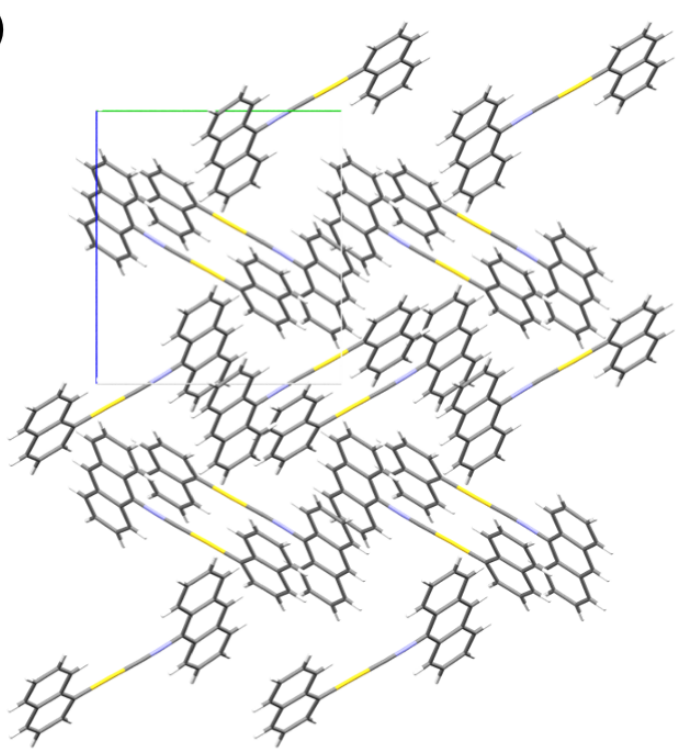

b)

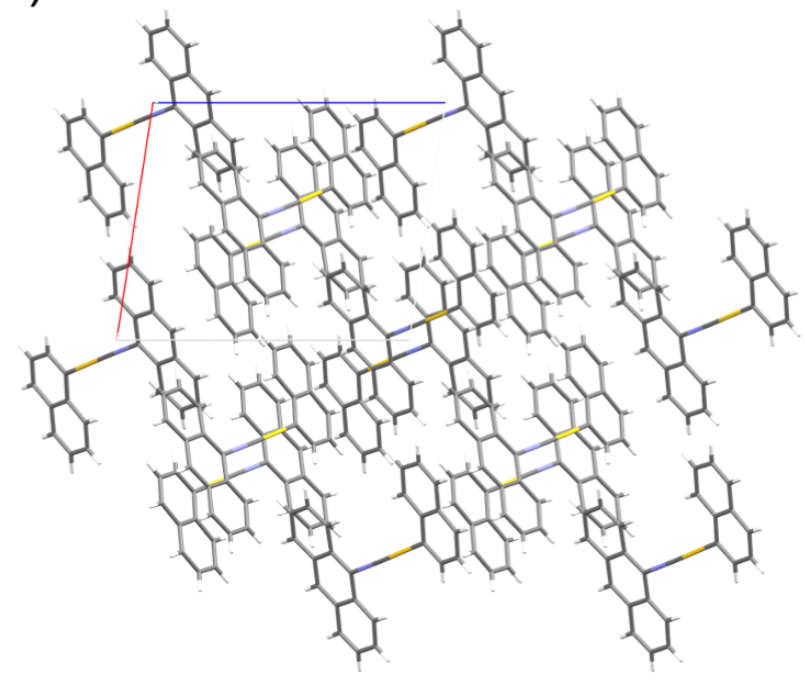

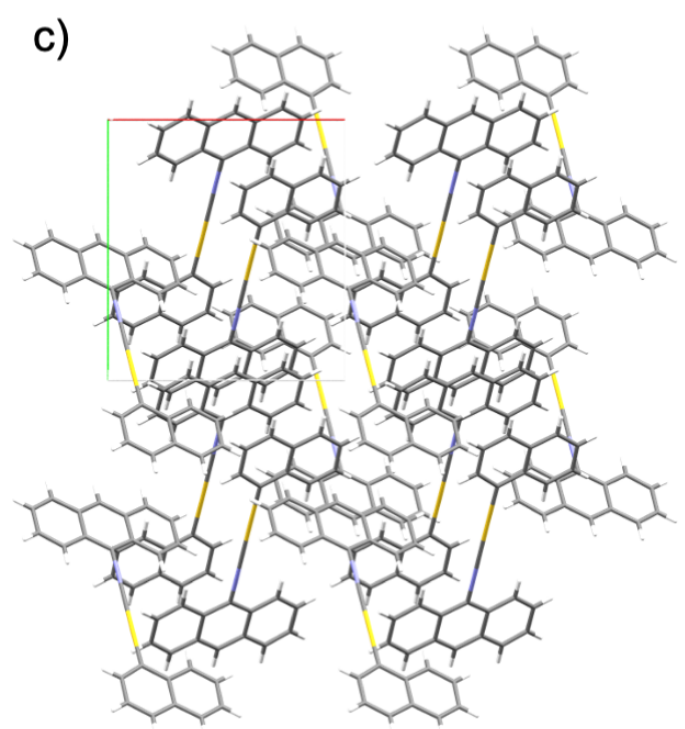

Figure S7. Single crystal structure of 1 at $20{ }^{\circ} \mathrm{C}$ viewed along a) $a$ axis, b) $b$ axis, and c) $c$ axis. 
a)

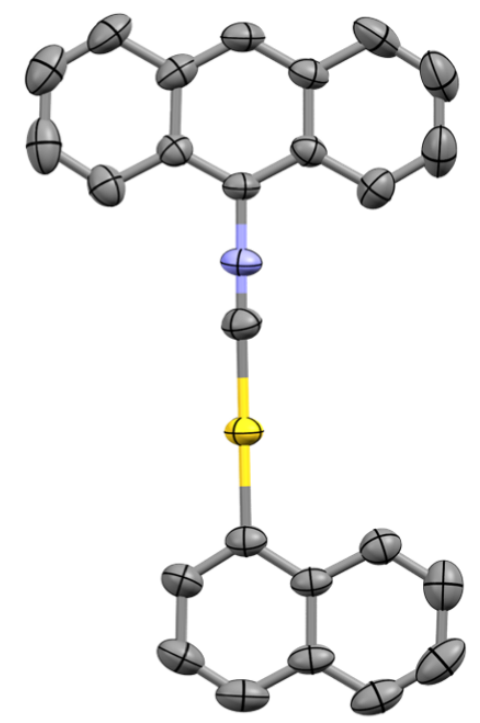

b)

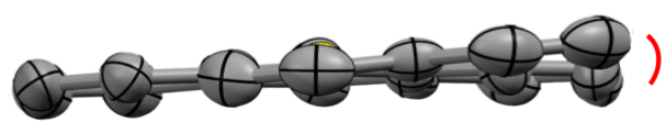

$5.1(3)^{\circ}$

Figure S8. a) Top view of single-crystal structure of 1 at $20^{\circ} \mathrm{C}$. b) Side view of single-crystal structure of $\mathbf{1}$ at $20^{\circ} \mathrm{C}$ showing the dihedral angle between the naphthalene and anthracene rings.

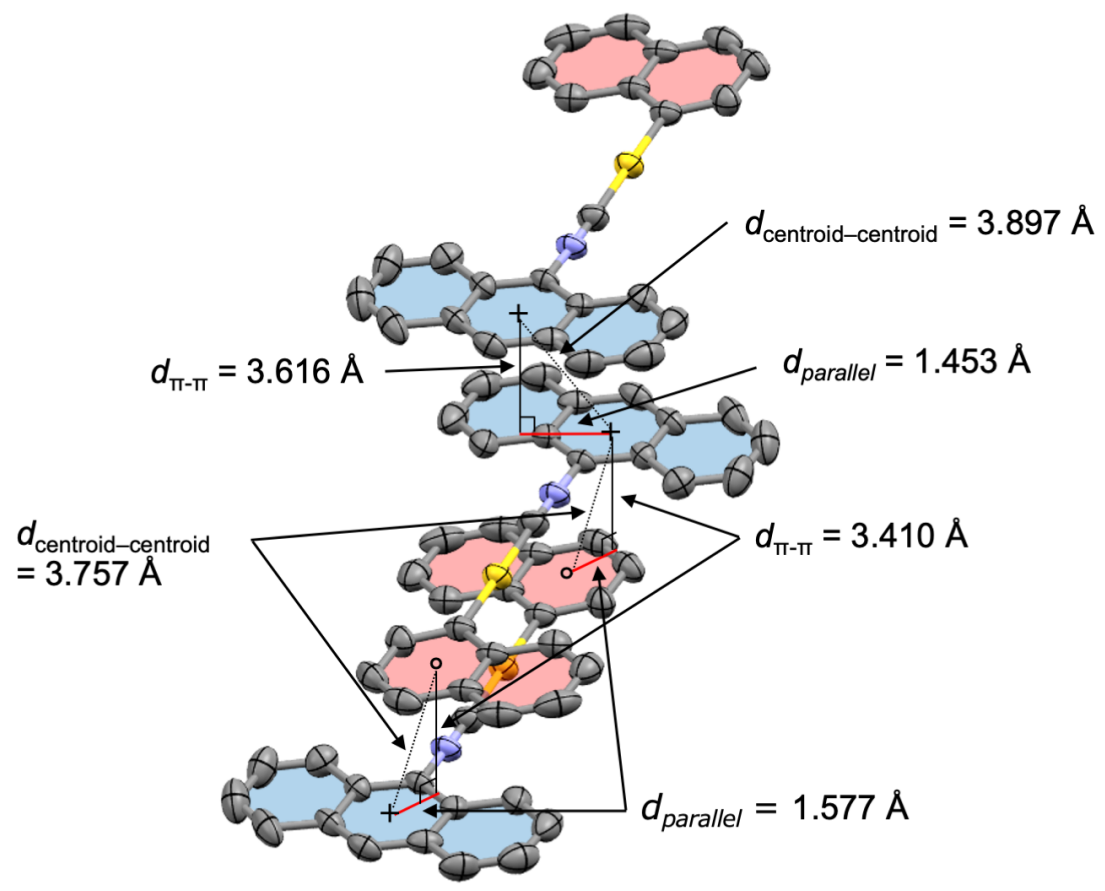

Figure S9. Single-crystal structure of 1 showing the lateral distance between the center of the anthryl group (depicted in "+") and the center of a 6-menbered ring within the naphthyl group (depicted in "०"). 
Preparation of the photodimer for single-crystal XRD analysis: the recrystallized sample of 1 prepared from its $\mathrm{CHCl}_{3}$ solution was put in Paratone oil and exposed to sunlight for a week. The photodimerized product $\mathbf{1}_{\text {PD }}$ was obtained as a colorless crystal.

a)

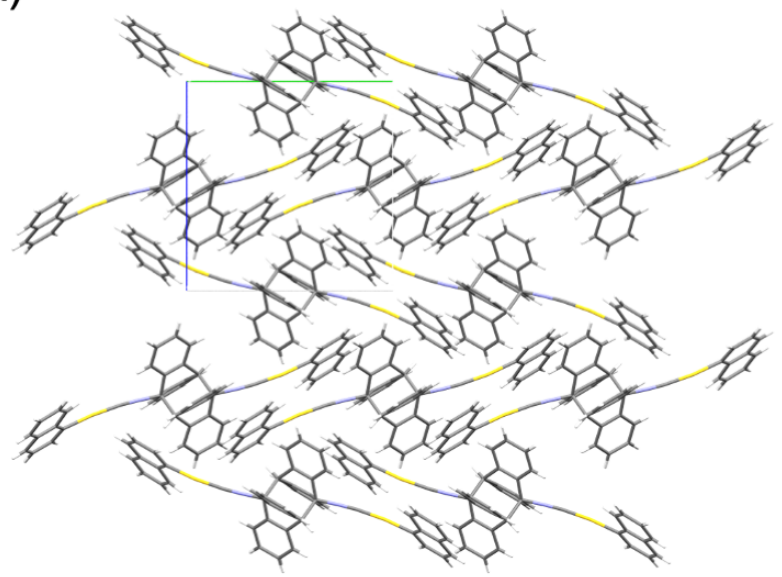

b)

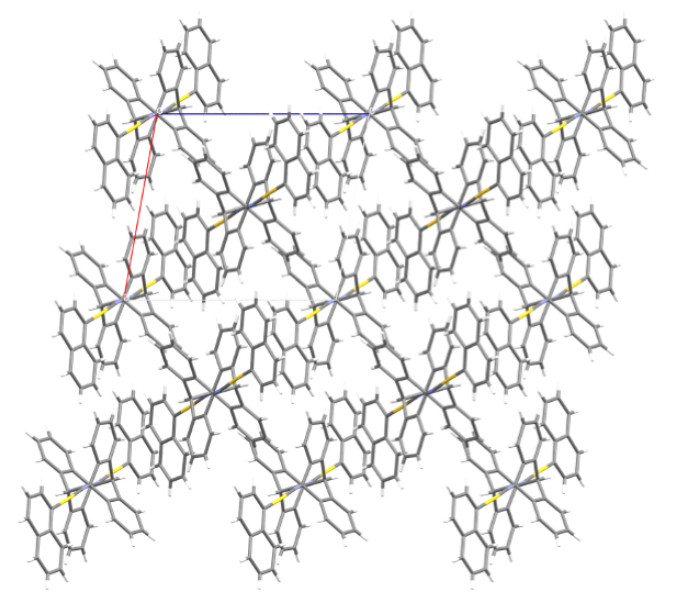

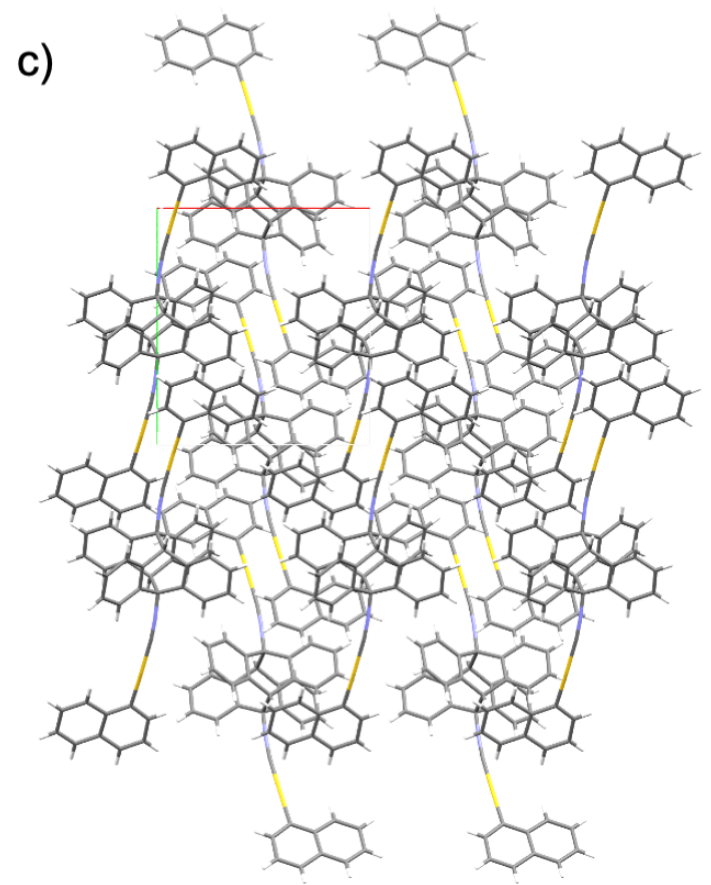

Figure S10. Single-crystal structure of $\mathbf{1}_{\mathbf{P D}}$ at $20^{\circ} \mathrm{C}$ viewed along a) $a$ axis, b) $b$ axis, and c) $c$ axis. 


\section{Photodimerization of 1 upon photoirradiation}

Preparation of the photodimer for NMR and HRMS: the recrystallized sample of $\mathbf{1}$ prepared from its $\mathrm{CHCl}_{3}$ solution was put in the vials and exposed to ultraviolet light $(365 \mathrm{~nm})$ for $3 \mathrm{~h}$ with manual stirring at roughly $30 \mathrm{~min}$ intervals. The photodimerized product $\mathbf{1}_{\mathbf{P D}}$ was obtained as a white solid.

1PD ESI-MS (m/z): [M+Na] $]^{+}$calcd. for $\mathrm{C}_{50} \mathrm{H}_{32} \mathrm{Au}_{2} \mathrm{~N}_{2} \mathrm{Na}$, 1077.17942; found, 1077.17618.

\section{a)}
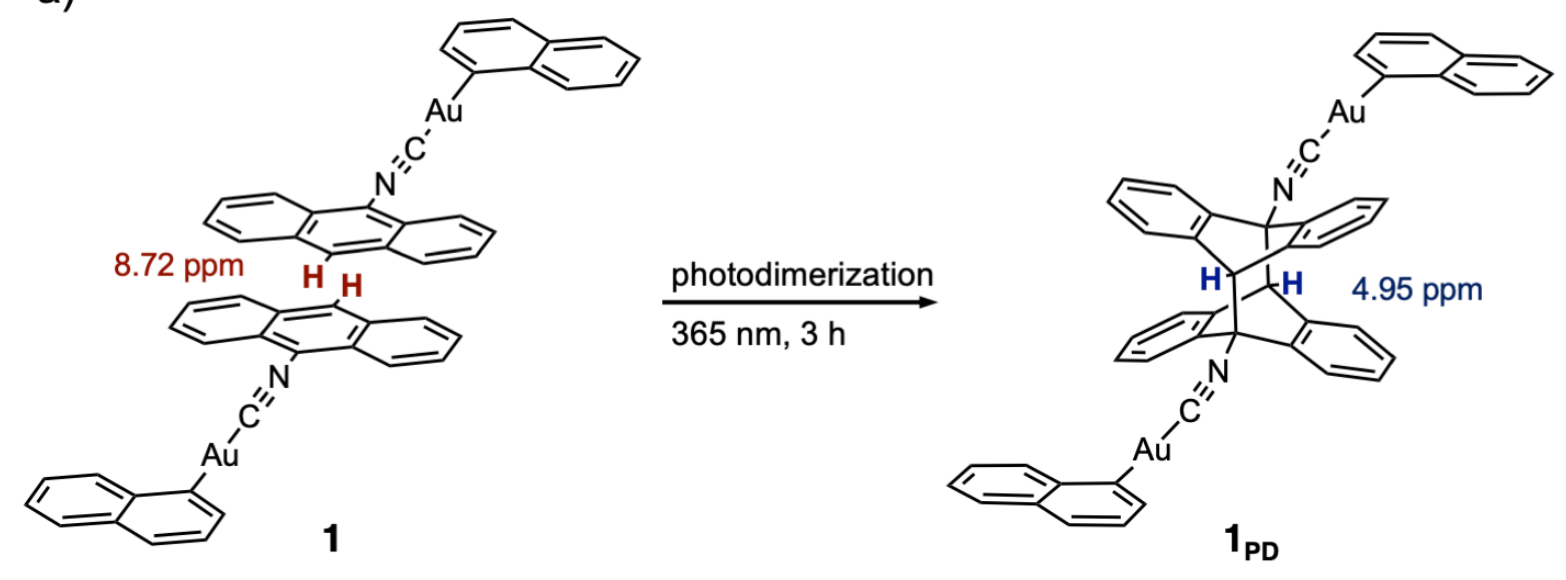

b)

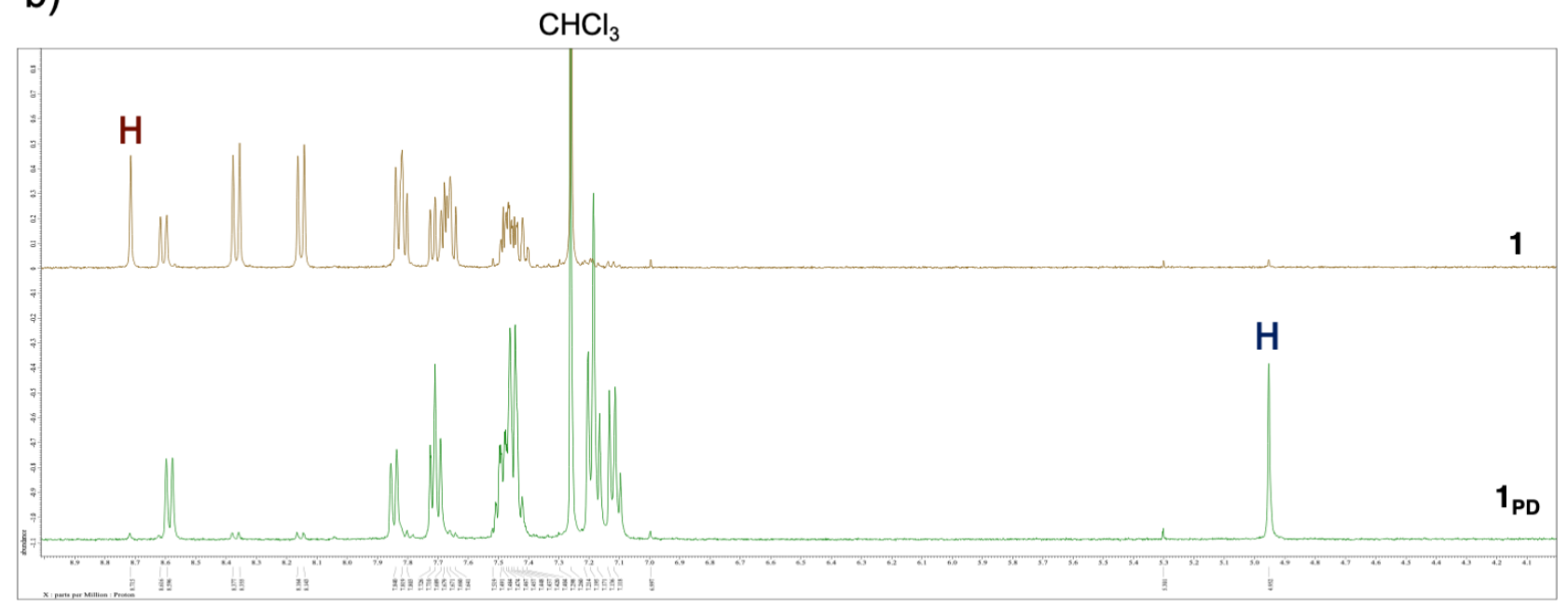

Figure S11. a) Scheme of photodimerization of 1 to give $\mathbf{1}_{\mathbf{P D}}$. b) Comparison of ${ }^{1} \mathrm{H}$ NMR spectra of 1 and $\mathbf{1}_{\mathbf{P D}}\left(400 \mathrm{MHz}, \mathrm{CDCl}_{3}\right)$. 


\section{Comparison of single-crystal structures of 1 and $1_{P D}$}

a)

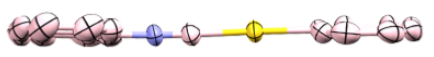

$48-0-0-8-25$

1

c)

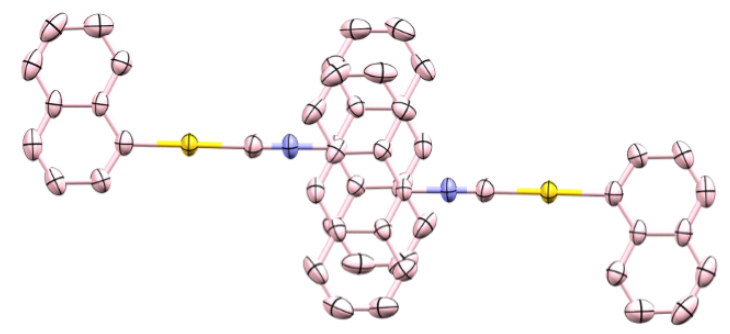

1 b)

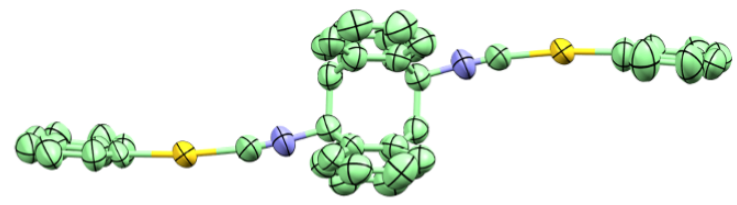

$1_{\mathrm{PD}}$

d)

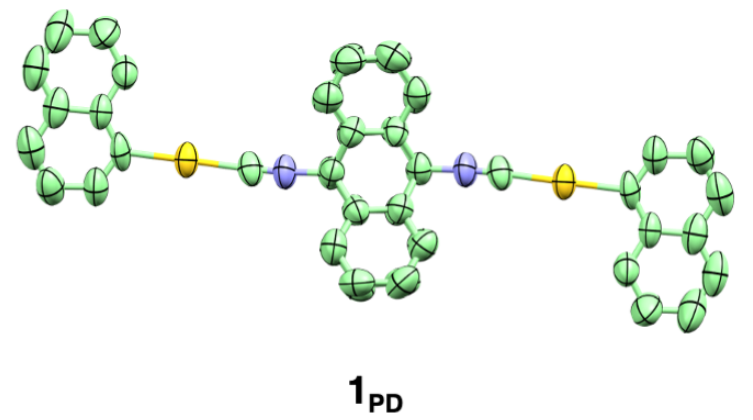

Figure S12. a) Side view and c) top view of single-crystal structures of $\pi$-stacked dimer of $\mathbf{1}$ at $20{ }^{\circ} \mathrm{C}$.

b) Side view and d) top view of single crystal structures of $\mathbf{1}_{\mathbf{P D}}$ at $20^{\circ} \mathrm{C}$. 


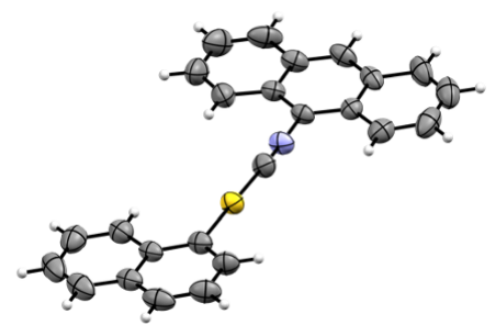

$20^{\circ} \mathrm{C}$

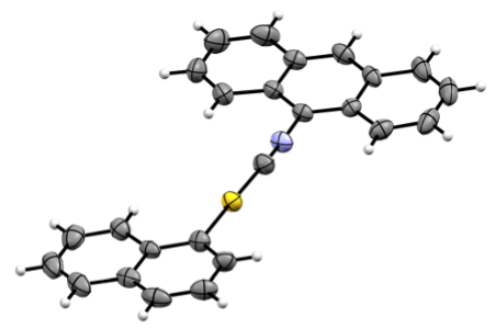

$-40^{\circ} \mathrm{C}$

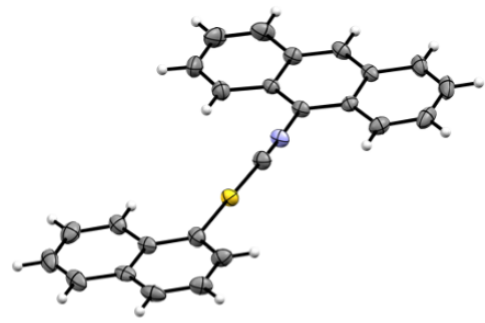

$-100^{\circ} \mathrm{C}$

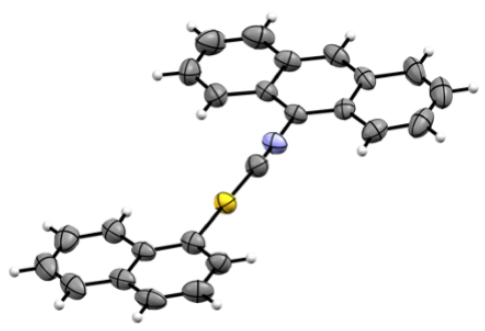

$0^{\circ} \mathrm{C}$

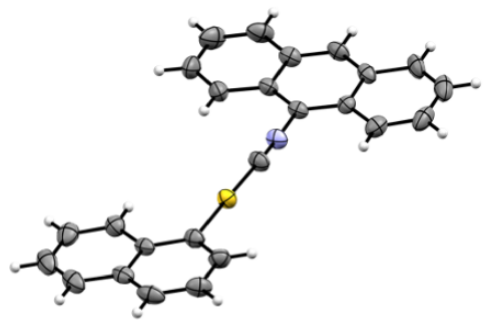

$-60^{\circ} \mathrm{C}$

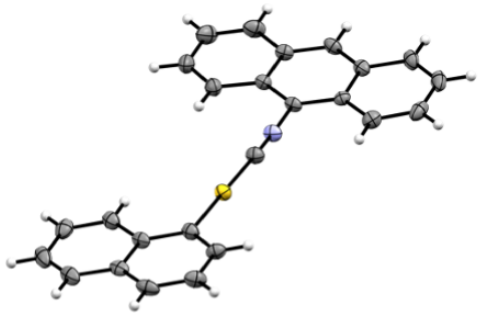

$-120^{\circ} \mathrm{C}$

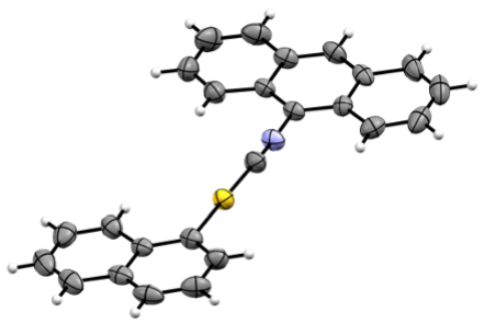

$-20^{\circ} \mathrm{C}$

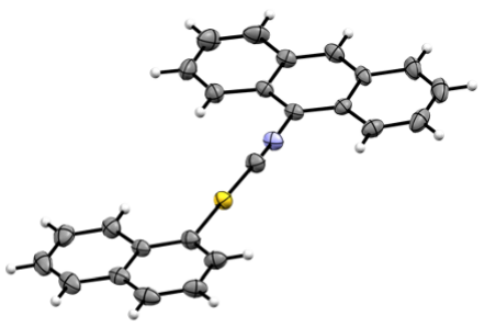

$-80^{\circ} \mathrm{C}$

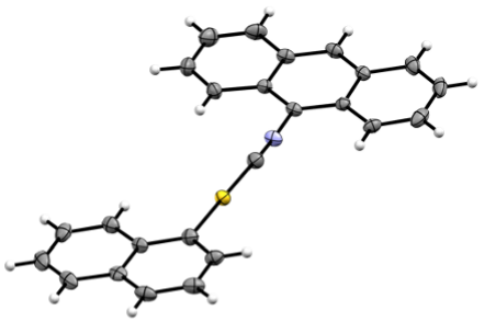

$-140^{\circ} \mathrm{C}$

Figure S13. Comparison of the monomers of 1 derived from the temperature-dependent single-crystal $\mathrm{XRD}$ measurements from $20^{\circ} \mathrm{C}$ to $-140{ }^{\circ} \mathrm{C}$. This figure indicates that the monomer conformations are almost unchanged upon temperature changes. 


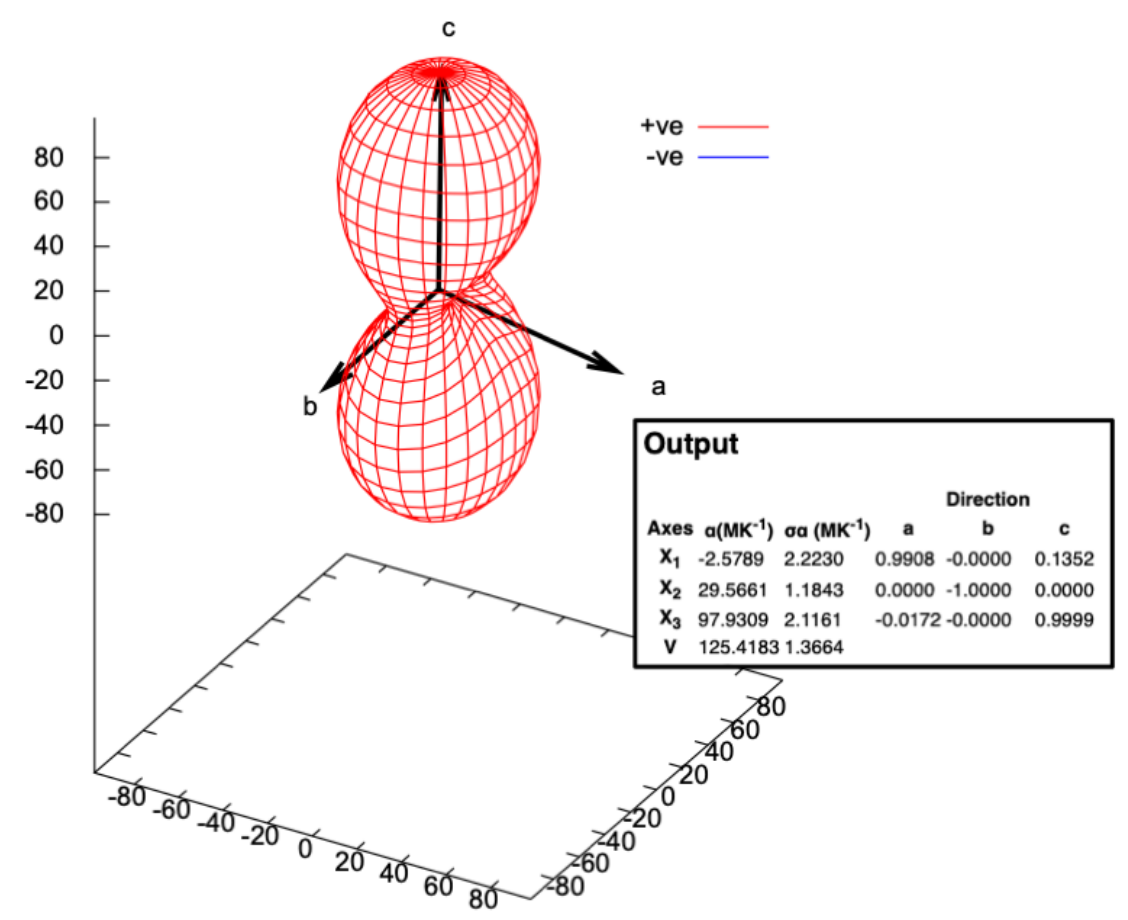

Figure S14. Thermal expansion coefficients and plot of the expansivity indicatrices of 1 calculated using the PASCal software. ${ }^{\mathrm{S} 9}$ The following input data were used for these analyses:

\section{Input}

$\begin{array}{llllllll}\text { Temp. } & \text { deviation } & a & b & c & \alpha & \beta & \gamma \\ 20 & 1 & 11.2334 & 12.1886 & 13.7168 & 90 & 98.7120 & 90 \\ 0 & 1 & 11.2374 & 12.1785 & 13.6868 & 90 & 98.7170 & 90 \\ -20 & 1 & 11.2405 & 12.1704 & 13.6580 & 90 & 98.7070 & 90 \\ -40 & 1 & 11.2441 & 12.1624 & 13.6315 & 90 & 98.7060 & 90 \\ -60 & 1 & 11.2440 & 12.1554 & 13.6015 & 90 & 98.6920 & 90 \\ -80 & 1 & 11.2448 & 12.1479 & 13.5770 & 90 & 98.6880 & 90 \\ -100 & 1 & 11.2414 & 12.1414 & 13.5495 & 90 & 98.650 & 90 \\ -120 & 1 & 11.2372 & 12.1360 & 13.5280 & 90 & 98.6350 & 90 \\ -140 & 1 & 11.2361 & 12.1310 & 13.5071 & 90 & 98.6270 & 90\end{array}$




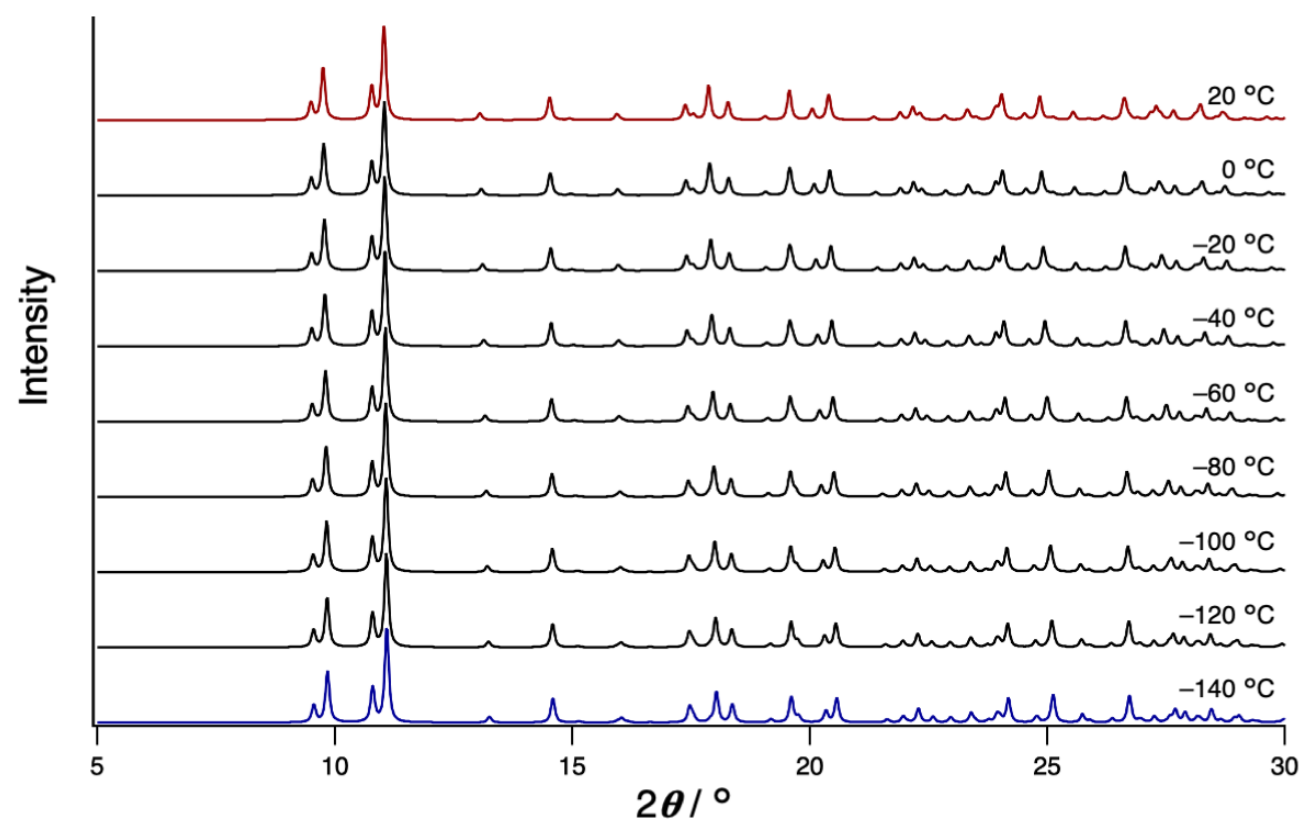

Figure S15. Simulated powder XRD patterns derived from the temperature-dependent single-crystal XRD measurements of 1 from $20^{\circ} \mathrm{C}$ to $-140{ }^{\circ} \mathrm{C}$. 


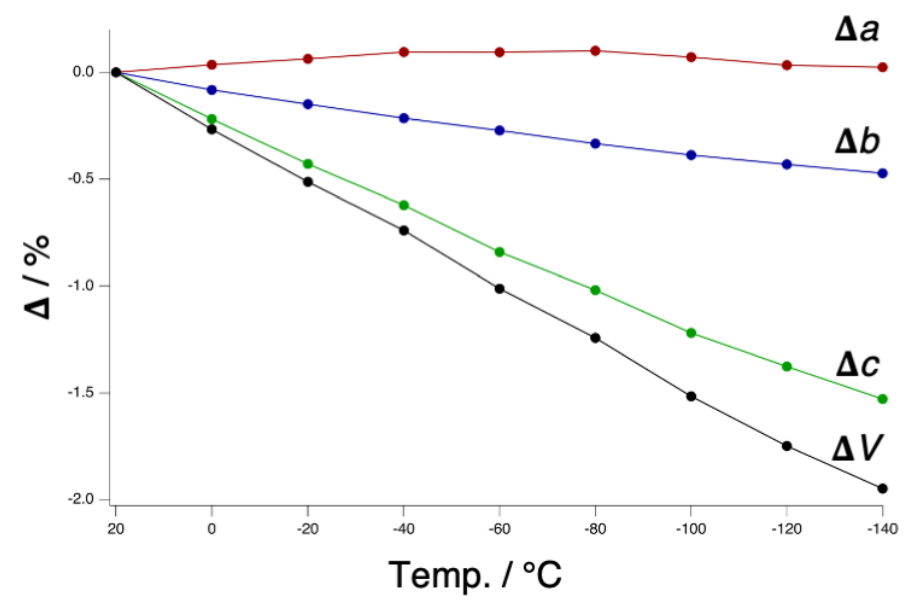

Figure S16. Plots of the changes in the crystallographic parameters $\Delta a, \Delta b, \Delta c$, and $\Delta V$ of 1 versus temperature. $\Delta a$ is defined as $\Delta a=a_{T}-a_{20}{ }^{\circ} \mathrm{C}$, in which $T$ is given temperature, $a_{T}$ and $\mathrm{a}_{20}{ }^{\circ} \mathrm{C}$ are values at temperature of $T$ and $20^{\circ} \mathrm{C}$, respectively. $\Delta b, \Delta c$, and $\Delta V$ are defined in the same manner. 


\section{DSC trace of 1}

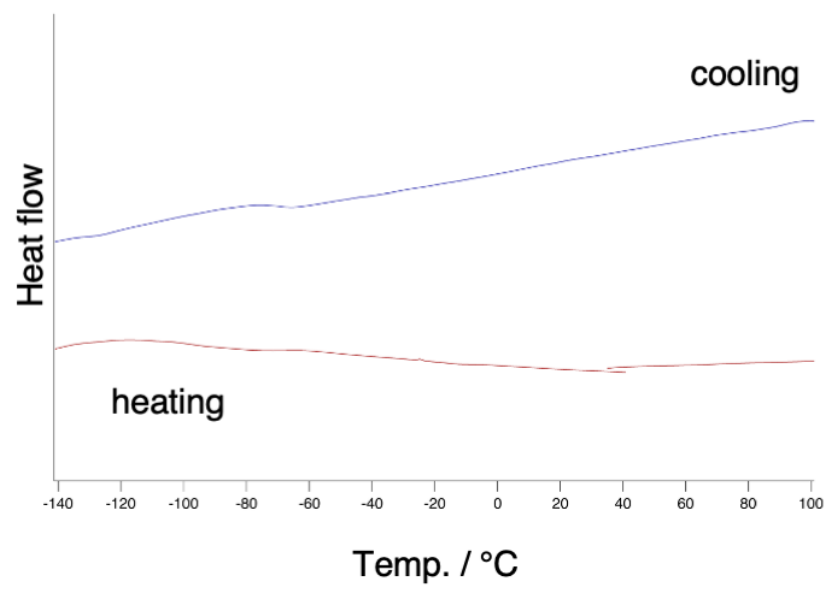

Figure S17. DSC traces of the crystals of $\mathbf{1}$ at cooling/heating rate of $5^{\circ} \mathrm{C} / \mathrm{min}$. 


\section{Photodimerization and DSC traces of 2 and 3}

Preparation of the photodimer for NMR and HRMS: The photodimerized product $\mathbf{2}_{\text {PD }}$ was obtained as a white solid according to the procedure similar to that described for preparation of $\mathbf{1}$ PD.

2 PD ESI-MS $(m / z)$ : $[\mathrm{M}+\mathrm{Na}]^{+}$calcd. for $\mathrm{C}_{50} \mathrm{H}_{32} \mathrm{Au}_{2} \mathrm{~N}_{2} \mathrm{Na}$, 1077.17942; found, 1077.17574.

a)
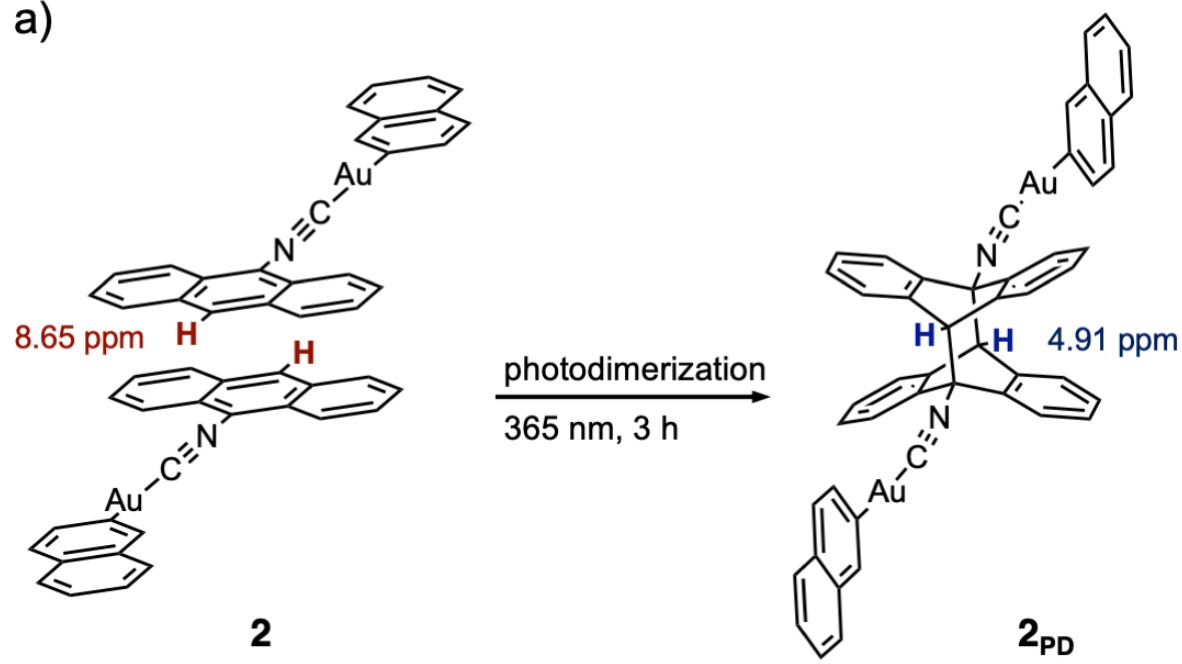

b)

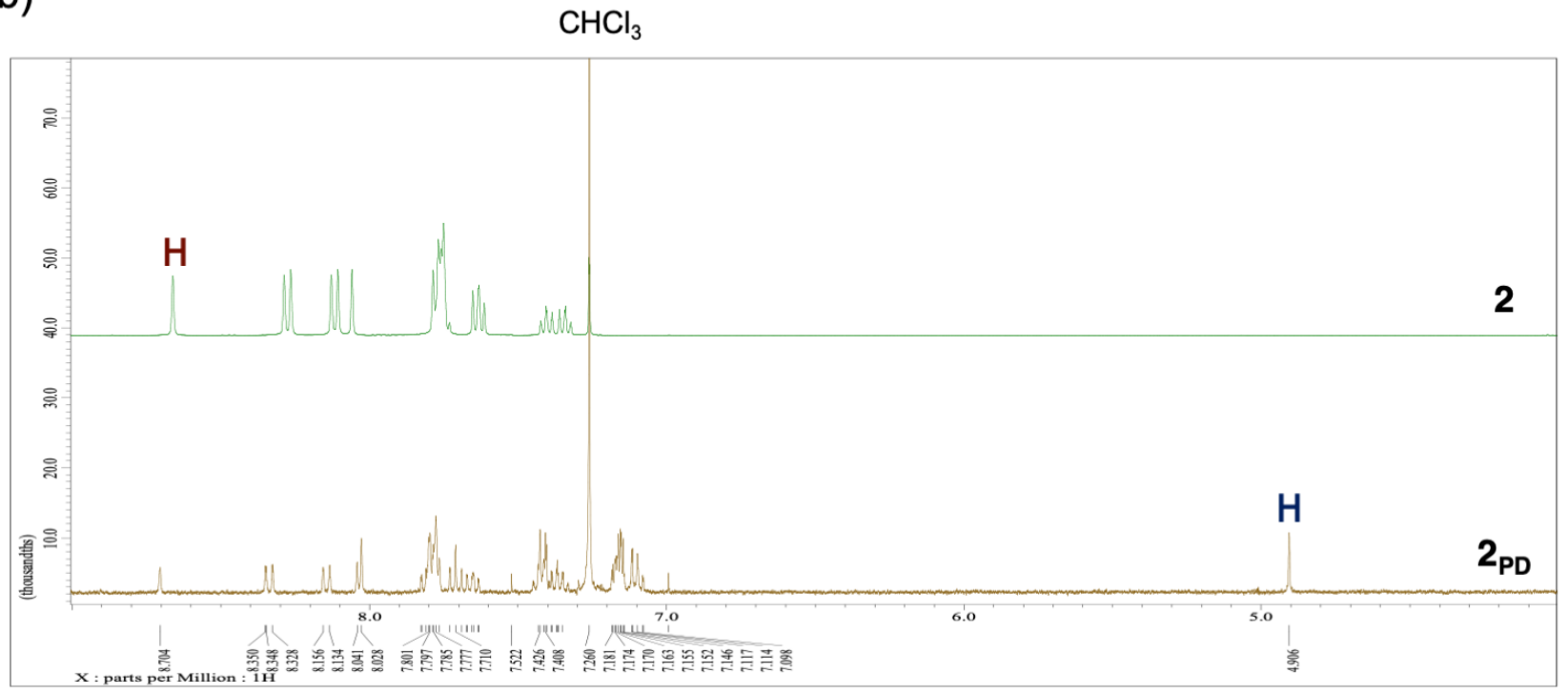

Figure S18. a) Scheme of photodimerization of $\mathbf{2}$ to give $\mathbf{2}_{\mathbf{P D}}$. b) Comparison of ${ }^{1} \mathrm{H}$ NMR spectra of 2 to $\mathbf{2}_{\mathbf{P D}}\left(400 \mathrm{MHz}, \mathrm{CDCl}_{3}\right)$. 
The photodimerized product $\mathbf{3}_{\text {PD }}$ was obtained as a white solid according to the procedure similar to that described for preparation of $\mathbf{1} \mathbf{P D}$.

3PD ESI-MS $(\mathrm{m} / z)$ : $[\mathrm{M}+\mathrm{Na}]^{+}$calcd. for $\mathrm{C}_{58} \mathrm{H}_{36} \mathrm{Au}_{2} \mathrm{~N}_{2} \mathrm{Na}, 1177.21072$; found, 1177.20687.

a)
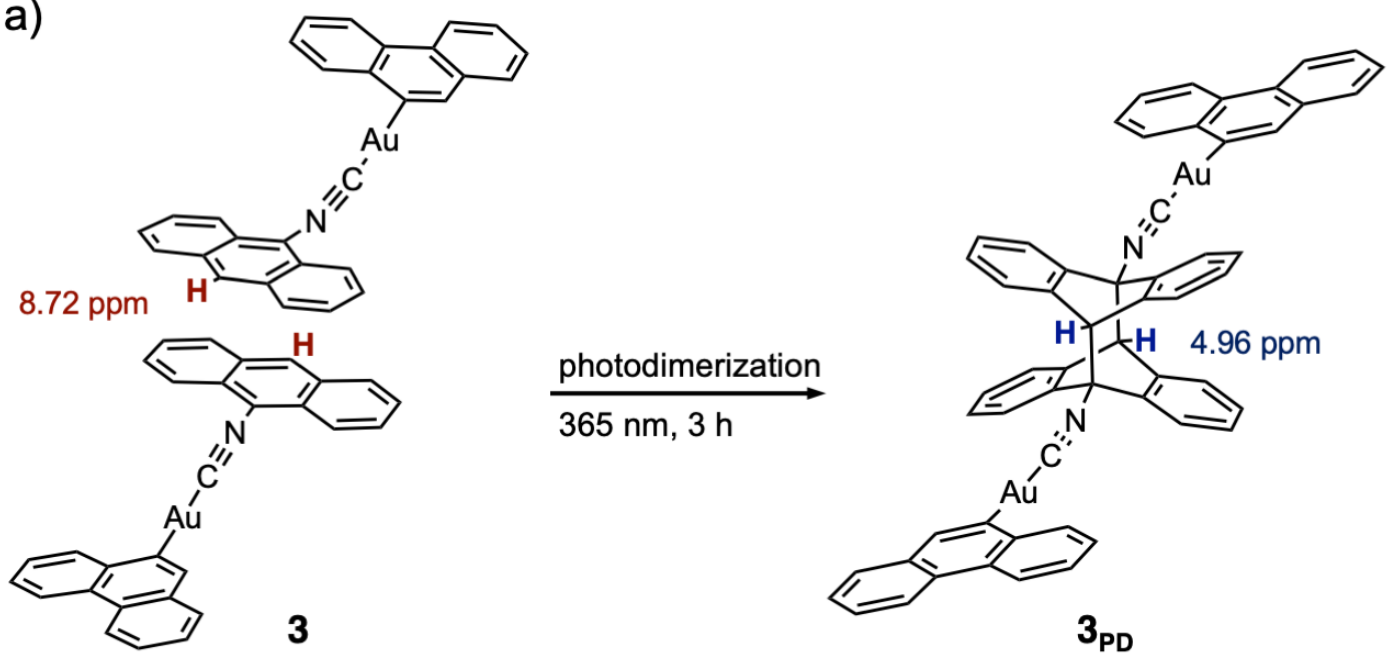

b)

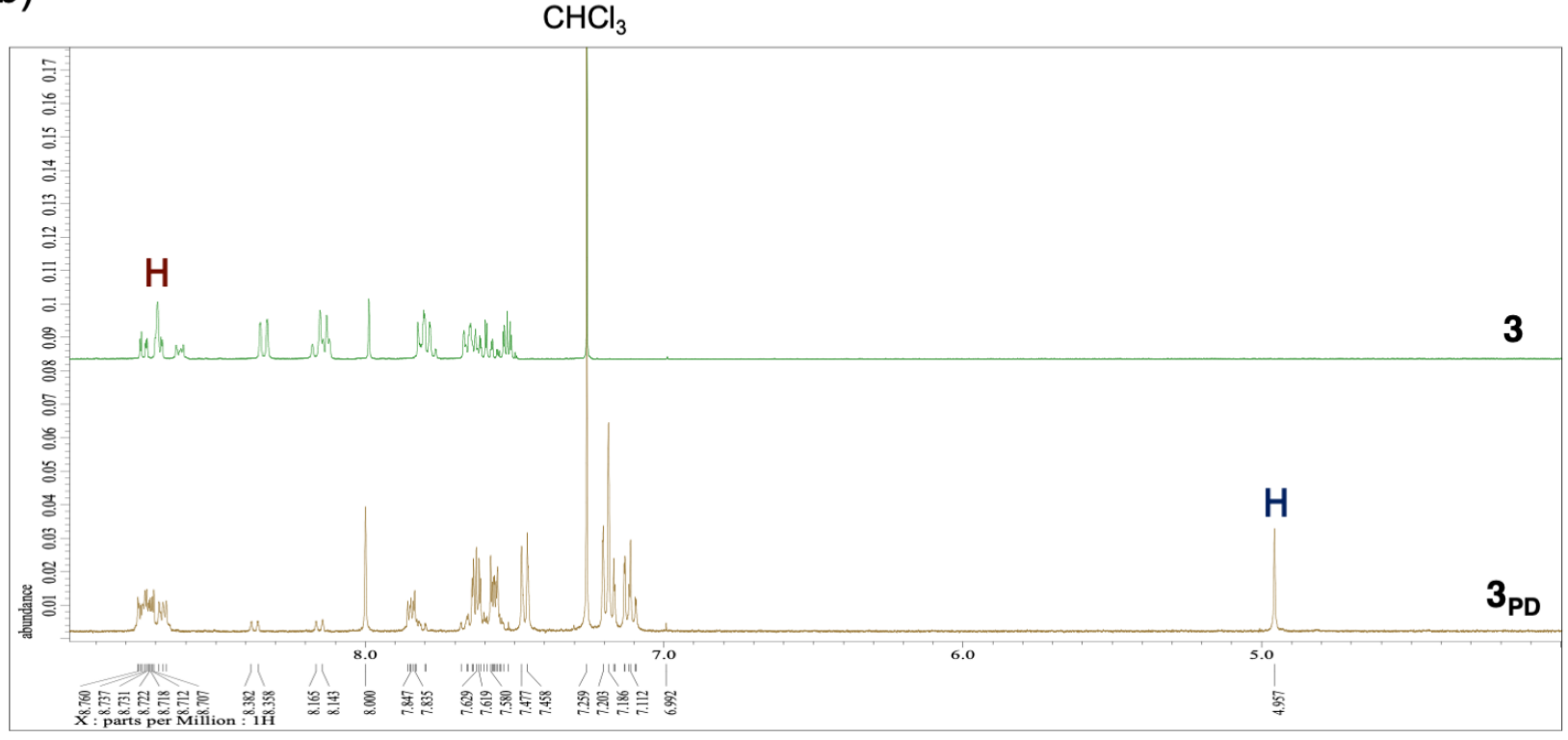

Figure S19. a) Scheme of photodimerization of $\mathbf{3}$ to give $\mathbf{3}_{\mathrm{PD}}$. b) Comparison of ${ }^{1} \mathrm{H}$ NMR spectra of $\mathbf{3}$ to $\mathbf{3}_{\mathbf{P D}}\left(400 \mathrm{MHz}, \mathrm{CDCl}_{3}\right)$. 
a)

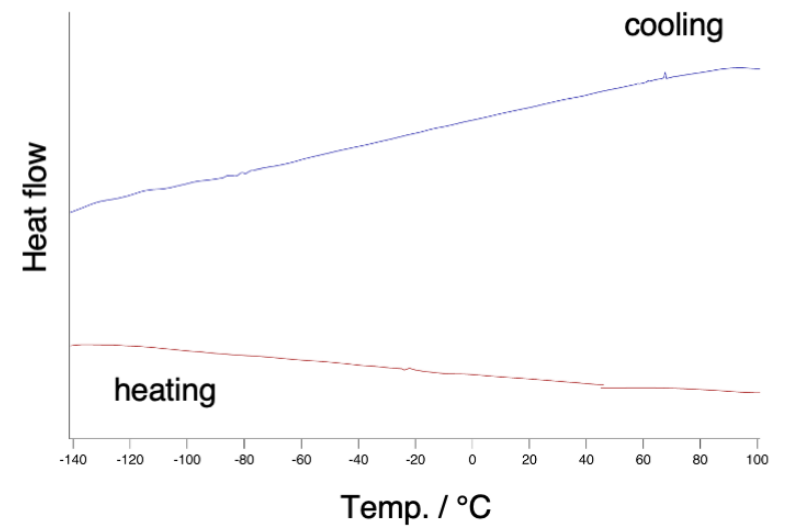

b)

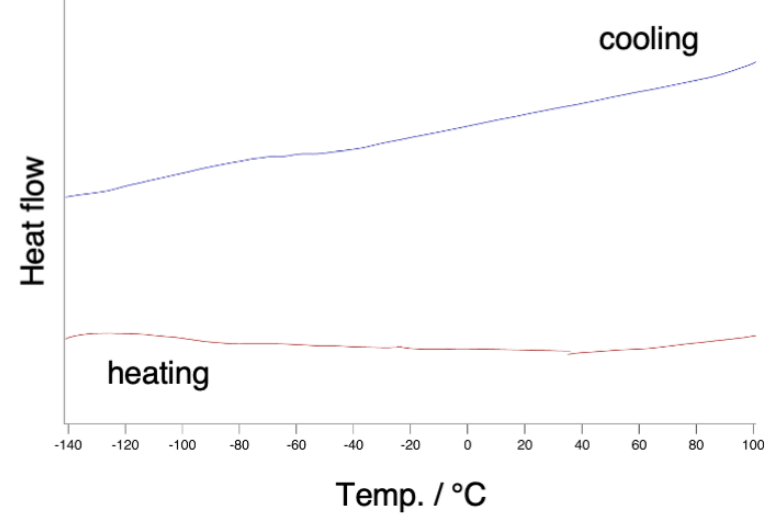

Figure S20. DSC traces of the crystals of a) 2 and b) 3 at cooling/heating rate of $5{ }^{\circ} \mathrm{C} / \mathrm{min}$. 
Table S2. Summary of X-ray crystallographic data for 3 at $20^{\circ} \mathrm{C}$ to $-140^{\circ} \mathrm{C}$.

\begin{tabular}{|c|c|c|c|c|c|}
\hline compound & 3 at $-140{ }^{\circ} \mathrm{C}$ & 3 at $-120^{\circ} \mathrm{C}$ & 3 at $-100^{\circ} \mathrm{C}$ & 3 at $-80^{\circ} \mathrm{C}$ & 3 at $-60^{\circ} \mathrm{C}$ \\
\hline CCDC Number & 2018154 & 2074064 & 2074065 & 2074066 & 2074067 \\
\hline Empirical Formula & $\mathrm{C}_{29} \mathrm{H}_{18} \mathrm{AuN}$ & $\mathrm{C}_{29} \mathrm{H}_{18} \mathrm{AuN}$ & $\mathrm{C}_{29} \mathrm{H}_{18} \mathrm{AuN}$ & $\mathrm{C}_{29} \mathrm{H}_{18} \mathrm{AuN}$ & $\mathrm{C}_{29} \mathrm{H}_{18} \mathrm{AuN}$ \\
\hline Formula Weight & 577.41 & 577.41 & 577.41 & 577.41 & 577.41 \\
\hline Crystal Size / mm & $0.20 \times 0.15 \times 0.10$ & $0.20 \times 0.15 \times 0.10$ & $0.20 \times 0.15 \times 0.10$ & $0.20 \times 0.15 \times 0.10$ & $0.20 \times 0.15 \times 0.10$ \\
\hline Crystal System & monoclinic & monoclinic & monoclinic & monoclinic & monoclinic \\
\hline$a / \AA$ & $14.9545(4)$ & $14.9566(3)$ & $14.9727(2)$ & $14.9831(2)$ & $14.9859(2)$ \\
\hline$b / \AA$ & $14.9500(3)$ & $14.9709(3)$ & $14.9960(2)$ & $15.0209(2)$ & $15.0490(2)$ \\
\hline$c / \AA$ & $18.5611(5)$ & $18.5727(4)$ & $18.5853(3)$ & $18.5995(2)$ & $18.6117(2)$ \\
\hline$\alpha 1^{\circ}$ & 90 & 90 & 90 & 90 & 90 \\
\hline$\beta /^{\circ}$ & $96.344(2)$ & $96.374(2)$ & $96.382(2)$ & $96.420(10)$ & $96.407(10)$ \\
\hline$\gamma /{ }^{\circ}$ & 90 & 90 & 90 & 90 & 90 \\
\hline$V / \AA^{3}$ & $4124.29(18)$ & $4132.98(15)$ & $4147.11(10)$ & $4159.75(9)$ & $4171.15(9)$ \\
\hline Space Group & $P 2_{1} / \mathrm{c}(\# 14)$ & $P 2_{1} / \mathrm{c}(\# 14)$ & $P 2_{1} / \mathrm{c}(\# 14)$ & $P 2_{1} / \mathrm{c}(\# 14)$ & $P 2_{1} / \mathrm{c}(\# 14)$ \\
\hline$Z$ value & 8 & 8 & 8 & 8 & 8 \\
\hline$D_{\text {calc }} / \mathrm{g} \mathrm{cm}^{-3}$ & 1.860 & 1.856 & 1.850 & 1.844 & 1.839 \\
\hline Temperature / K & $133(2)$ & $153(2)$ & $173(2)$ & $193(2)$ & $213(2)$ \\
\hline $2 \theta_{\max } /^{\circ}$ & 136.474 & 136.486 & 136.498 & 136.494 & 136.488 \\
\hline$\mu / \mathrm{mm}^{-1}$ & $13.518\left(\mathrm{Cu} \mathrm{K}_{\alpha}\right)$ & $13.490\left(\mathrm{Cu} \mathrm{K}_{\alpha}\right)$ & $13.444\left(\mathrm{Cu} \mathrm{K}_{\alpha}\right)$ & $13.403\left(\mathrm{Cu} \mathrm{K}_{\alpha}\right)$ & $13.366\left(\mathrm{Cu} \mathrm{K}_{\alpha}\right)$ \\
\hline No. of Reflections & $\begin{array}{l}\text { Total: } 35726 \\
\text { Unique: } 7543 \\
R_{\text {int }}=0.0915\end{array}$ & $\begin{array}{l}\text { Total: } 35596 \\
\text { Unique: } 7561 \\
R_{\text {int }}=0.0790\end{array}$ & $\begin{array}{l}\text { Total: } 35239 \\
\text { Unique: } 7592 \\
R_{\text {int }}=0.0770\end{array}$ & $\begin{array}{l}\text { Total: } 37134 \\
\text { Unique: } 7620 \\
R_{\text {int }}=0.0765\end{array}$ & $\begin{array}{l}\text { Total: } 37604 \\
\text { Unique: } 7645 \\
R_{\text {int }}=0.0754\end{array}$ \\
\hline$R_{1}^{\mathrm{a}}$ & 0.0464 & 0.0369 & 0.0364 & 0.0359 & 0.0364 \\
\hline$w R_{2}{ }^{\mathrm{b}}$ & 0.1149 & 0.0955 & 0.0923 & 0.0905 & 0.0935 \\
\hline $\mathrm{GOF}^{c}$ & 1.051 & 1.028 & 1.030 & 1.021 & 1.037 \\
\hline Max./Mini. peak $I / \AA^{3}$ & $1.79 \mathrm{e}^{-/-1.56 \mathrm{e}^{-}}$ & $1.23 \mathrm{e}^{-/}-1.15 \mathrm{e}^{-}$ & $0.98 \mathrm{e}^{-/-0.99 \mathrm{e}^{-}}$ & $0.94 \mathrm{e}^{-/-0.88 \mathrm{e}^{-}}$ & $1.28 \mathrm{e}^{-/-1.00 \mathrm{e}^{-}}$ \\
\hline compound & 3 at $-40{ }^{\circ} \mathrm{C}$ & 3 at $-20^{\circ} \mathrm{C}$ & 3 at $0{ }^{\circ} \mathrm{C}$ & 3 at $20^{\circ} \mathrm{C}$ & \\
\hline CCDC Number & 2074068 & 2074069 & 2074070 & 2018155 & \\
\hline Empirical Formula & $\mathrm{C}_{29} \mathrm{H}_{18} \mathrm{AuN}$ & $\mathrm{C}_{29} \mathrm{H}_{18} \mathrm{AuN}$ & $\mathrm{C}_{29} \mathrm{H}_{18} \mathrm{AuN}$ & $\mathrm{C}_{29} \mathrm{H}_{18} \mathrm{AuN}$ & \\
\hline Formula Weight & 577.41 & 577.41 & 577.41 & 577.41 & \\
\hline Crystal Size / mm & $0.20 \times 0.15 \times 0.10$ & $0.20 \times 0.15 \times 0.10$ & $0.20 \times 0.15 \times 0.10$ & $0.20 \times 0.15 \times 0.10$ & \\
\hline Crystal System & monoclinic & monoclinic & monoclinic & monoclinic & \\
\hline$a / \AA$ & $14.9892(2)$ & $14.9922(2)$ & $14.9939(2)$ & $14.9954(2)$ & \\
\hline$b / \AA$ & $15.0812(2)$ & $15.1115(2)$ & $15.1435(2)$ & $15.1748(2)$ & \\
\hline$c / \AA$ & $18.6260(2)$ & $18.6420(2)$ & $18.6580(2)$ & $18.6772(2)$ & \\
\hline$\alpha 1^{\circ}$ & 90 & 90 & 90 & 90 & \\
\hline$\beta 1^{\circ}$ & $96.429(10)$ & $96.452(10)$ & $96.449(10)$ & $96.456(10)$ & \\
\hline$\gamma /^{\circ}$ & 90 & 90 & 90 & 90 & \\
\hline$V / \AA^{3}$ & $4184.02(9)$ & $4196.68(9)$ & $4209.68(9)$ & $4223.09(9)$ & \\
\hline Space Group & $P 2_{1} / \mathrm{c}(\# 14)$ & $P 2_{1} / \mathrm{c}(\# 14)$ & $P 2_{1} / \mathrm{c}(\# 14)$ & $P 2_{1} / \mathrm{c}(\# 14)$ & \\
\hline$Z$ value & 8 & 8 & 8 & 8 & \\
\hline$D_{\text {calc }} / \mathrm{g} \mathrm{cm}^{-3}$ & 1.833 & 1.828 & 1.822 & 1.816 & \\
\hline Temperature / K & $233(2)$ & $253(2)$ & $273(2)$ & 293(2) & \\
\hline $2 \theta_{\max } 1^{\circ}$ & 136.504 & 136.47 & 136.498 & 136.502 & \\
\hline$\mu / \mathrm{mm}^{-1}$ & $13.325\left(\mathrm{Cu} \mathrm{K}_{\alpha}\right)$ & $13.285\left(\mathrm{Cu} \mathrm{K}_{\alpha}\right)$ & $13.244\left(\mathrm{Cu} \mathrm{K}_{\alpha}\right)$ & $13.202\left(\mathrm{Cu} \mathrm{K}_{\alpha}\right)$ & \\
\hline No. of Reflections & $\begin{array}{l}\text { Total: } 37428 \\
\text { Unique: } 7666 \\
R_{\text {int }}=0.0727\end{array}$ & $\begin{array}{l}\text { Total: } 38633 \\
\text { Unique: } 7685 \\
R_{\text {int }}=0.0730\end{array}$ & $\begin{array}{l}\text { Total: } 38464 \\
\text { Unique: } 7712 \\
R_{\text {int }}=0.0708\end{array}$ & $\begin{array}{l}\text { Total: } 37677 \\
\text { Unique: } 7736 \\
R_{\text {int }}=0.0707\end{array}$ & \\
\hline$R_{1}{ }^{\mathrm{a}}$ & 0.0347 & 0.0361 & 0.0361 & 0.0368 & \\
\hline$w R_{2}{ }^{\mathrm{b}}$ & 0.0877 & 0.0941 & 0.0953 & 0.0969 & \\
\hline $\mathrm{GOF}^{c}$ & 1.043 & 1.033 & 1.032 & 1.034 & \\
\hline Max./Mini. peak $I / \AA^{3}$ & $1.15 \mathrm{e}^{-/-1.00 \mathrm{e}^{-}}$ & $1.13 \mathrm{e}^{-/}-0.95 \mathrm{e}^{-}$ & $1.18 \mathrm{e}^{-/-1.07 \mathrm{e}^{-}}$ & $0.94 \mathrm{e}^{-/-1.07 \mathrm{e}^{-}}$ & \\
\hline
\end{tabular}

[a] For data with $I>2.00 \sigma(I)$. [b] For all reflection data. [c] Goodness of Fit. 


\section{Single-crystal XRD structure analyses of 3}

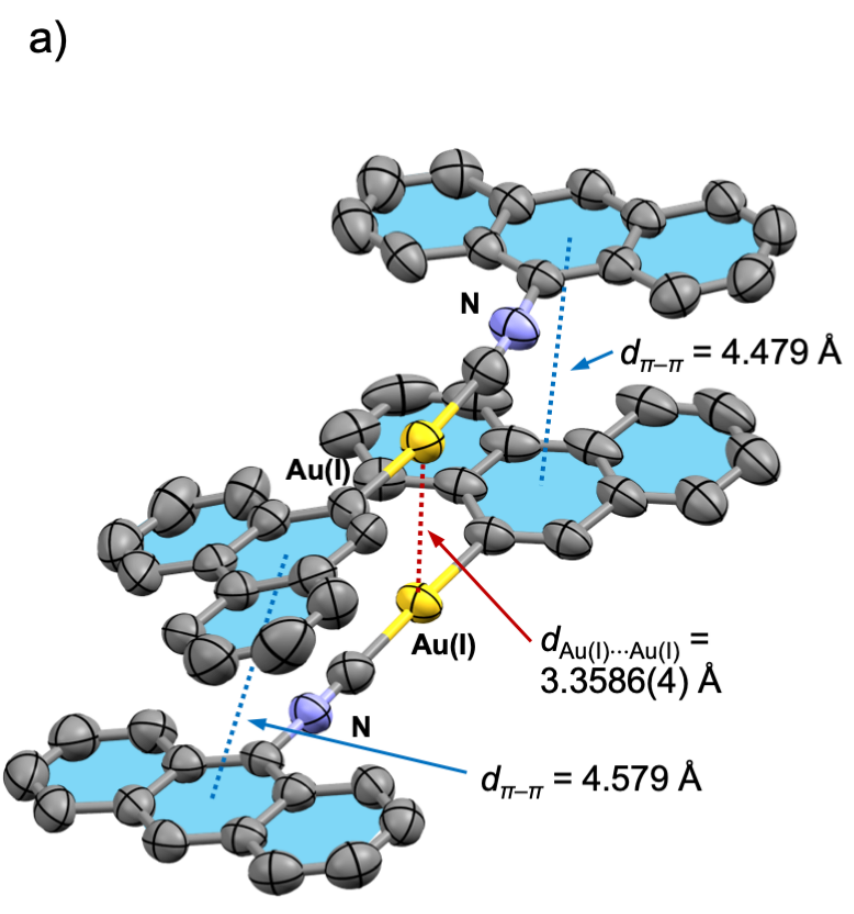

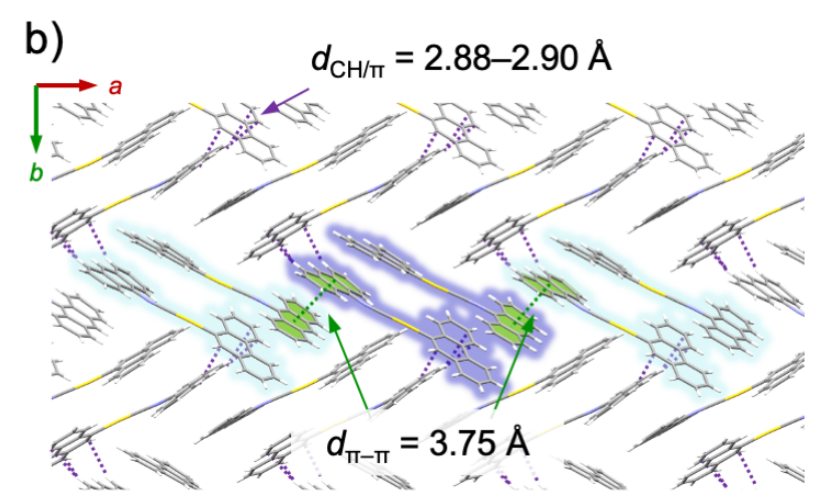

c)

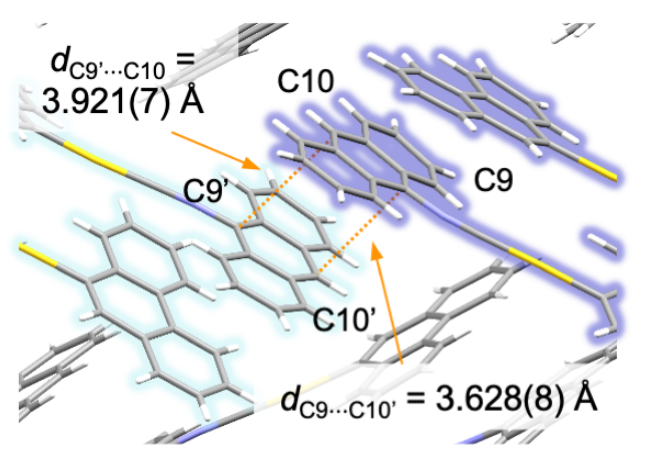

Figure S21. a) ORTEP drawing of the $\pi$-stacked dimer of 3 at $20^{\circ} \mathrm{C}$. Hydrogen atoms are omitted for clarity. b) Herringbone structure of $\mathbf{3}$ within the (001) plane. Three $\pi$-stacked dimers are highlighted in cyan and purple. c) Packing structure of 3 . Two adjacent $\pi$-stacked dimers are highlighted in cyan and purple. 
a)

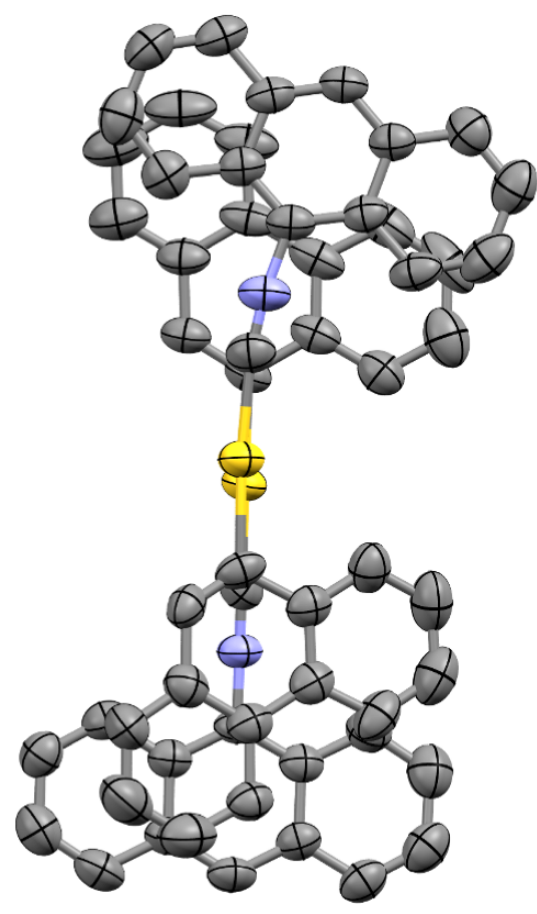

b)

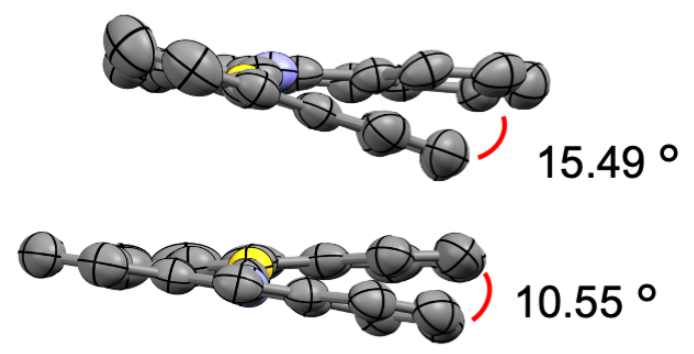

Figure S22. a) Top view of single-crystal structure of $\mathbf{3}$ at $20{ }^{\circ} \mathrm{C}$. b) Side view of single-crystal structure of $\mathbf{3}$ at $20^{\circ} \mathrm{C}$ showing the dihedral angle between the naphthalene and anthracene rings. 
a)

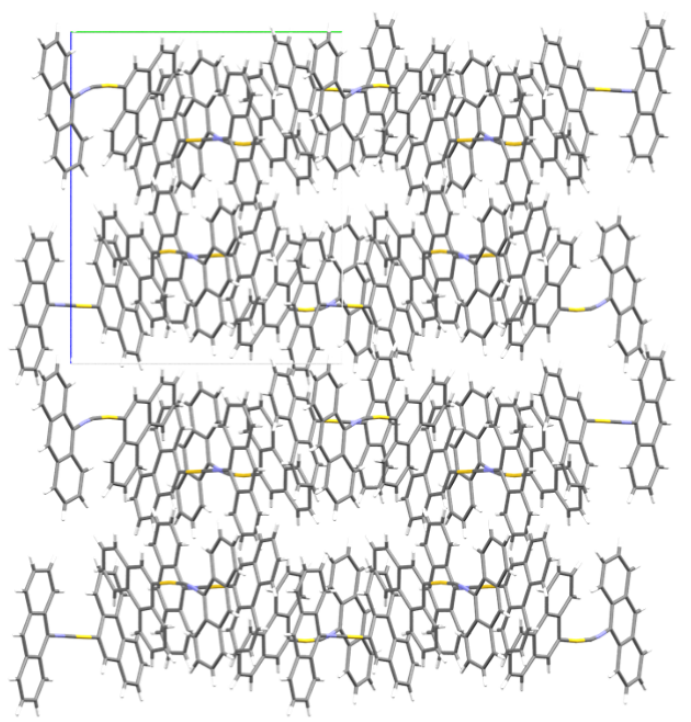

b)

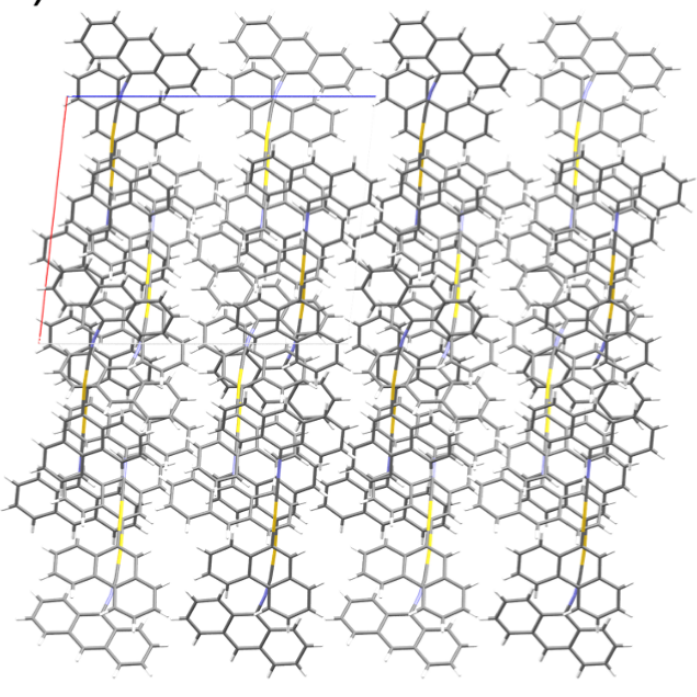

c)

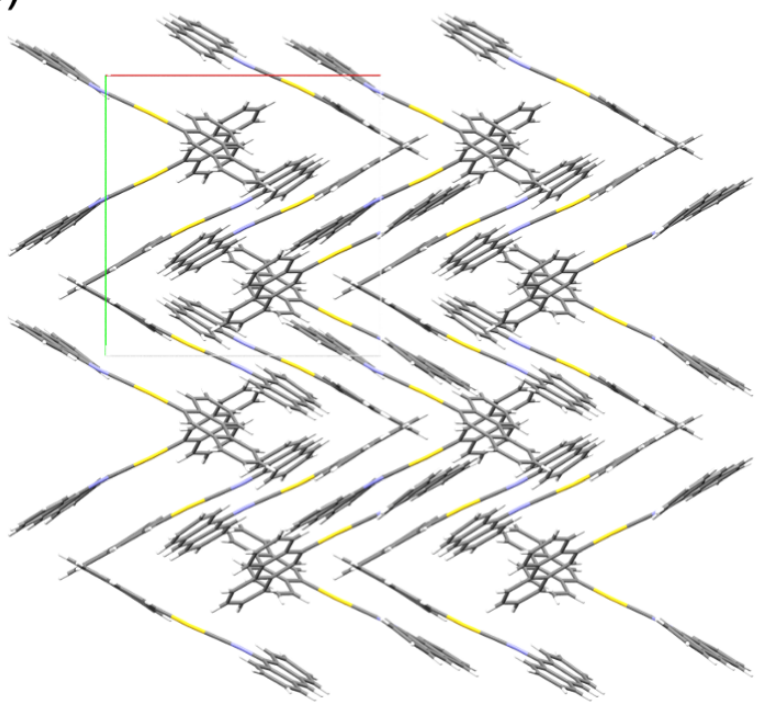

Figure S23. Single-crystal structure of $\mathbf{3}$ at $20{ }^{\circ} \mathrm{C}$ viewed along a) $a$ axis, b) $b$ axis, and c) $c$ axis. 

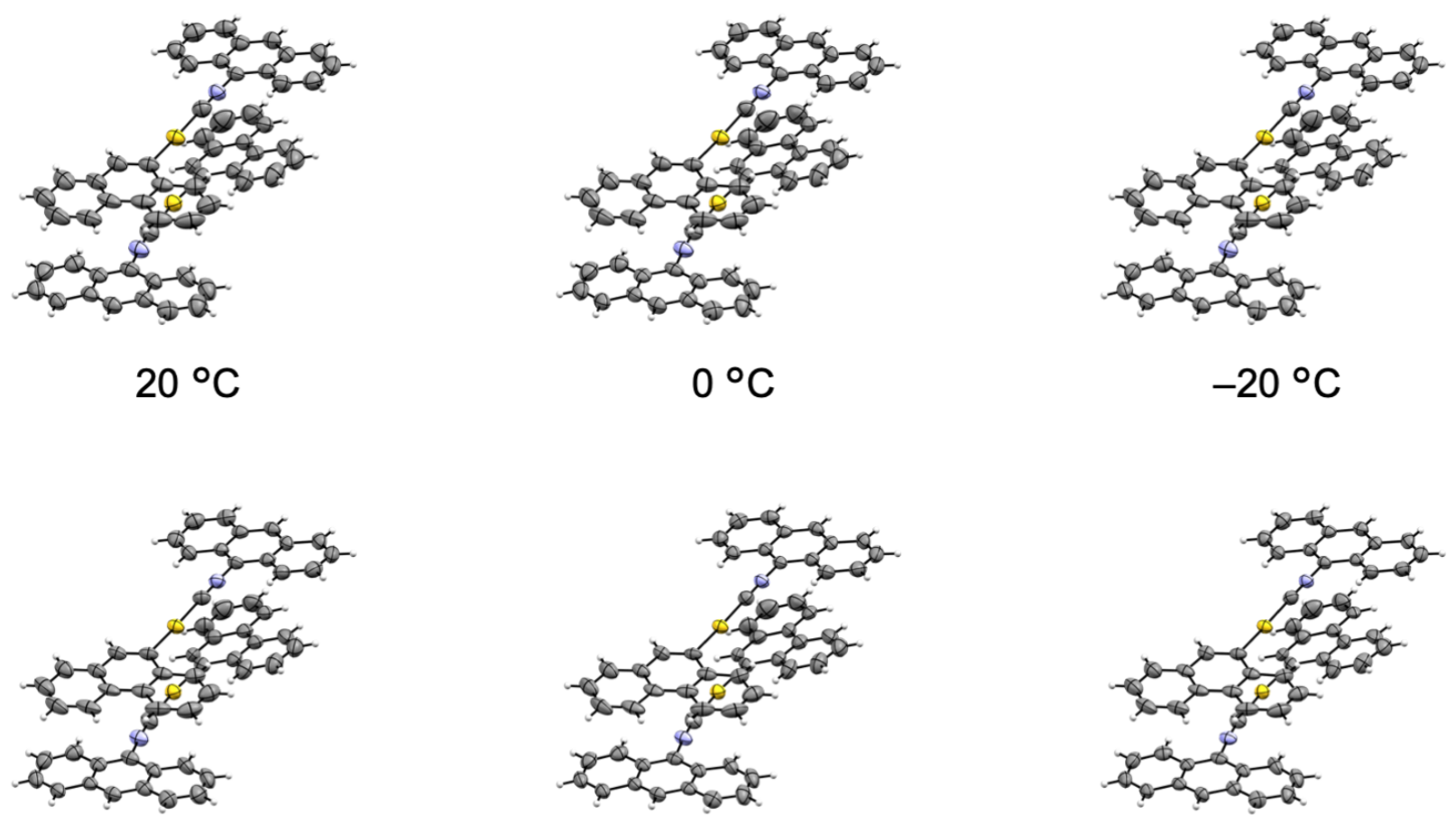

$-40^{\circ} \mathrm{C}$
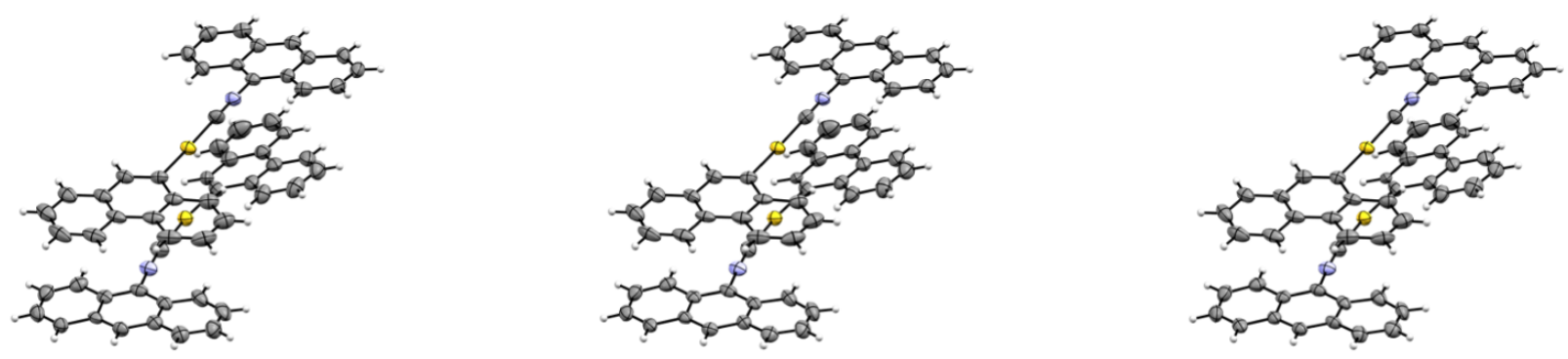

$-100^{\circ} \mathrm{C}$

$-120^{\circ} \mathrm{C}$

$-140^{\circ} \mathrm{C}$

Figure S24. Comparison of the $\pi$-stacked dimer of $\mathbf{3}$ derived from the temperature-dependent singlecrystal XRD measurements from $20^{\circ} \mathrm{C}$ to $-140{ }^{\circ} \mathrm{C}$. This figure indicates that the $\pi$-stacked dimers are almost unchanged upon temperature changes. 


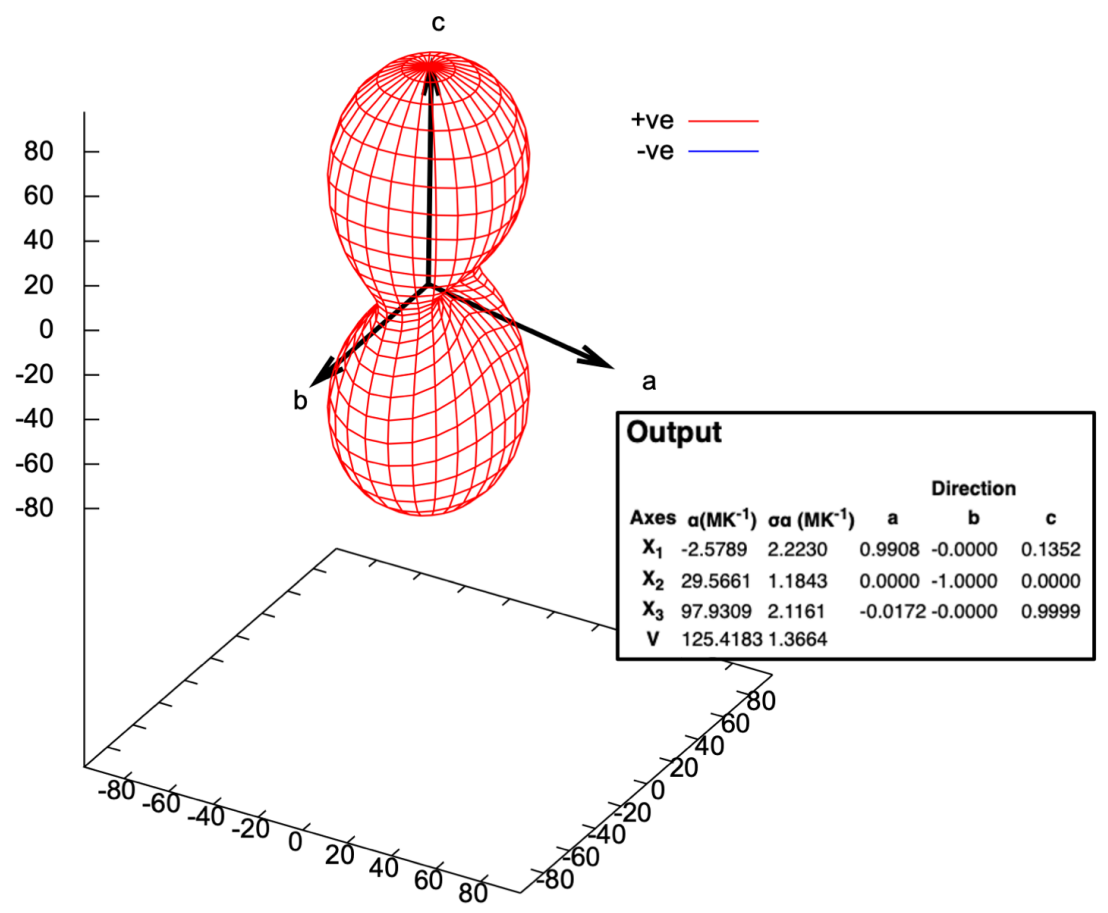

Figure S25. Thermal expansion coefficients and plot of the expansivity indicatrices of $\mathbf{3}$ calculated using the PASCal software ${ }^{\mathrm{S} 69}$ and the following input data:

\section{Input}

$\begin{array}{llllllll}\text { Temp. } & \text { deviation } & a & b & c & \alpha & \beta & \gamma \\ 20 & 1 & 14.9954 & 15.1748 & 18.6772 & 90 & 96.456 & 90 \\ 0 & 1 & 14.9939 & 15.1435 & 18.6580 & 90 & 96.449 & 90 \\ -20 & 1 & 14.9922 & 15.1115 & 18.6420 & 90 & 96.452 & 90 \\ -40 & 1 & 14.9892 & 15.0812 & 18.6260 & 90 & 96.429 & 90 \\ -60 & 1 & 14.9859 & 15.0490 & 18.6117 & 90 & 96.407 & 90 \\ -80 & 1 & 14.9831 & 15.0209 & 18.5995 & 90 & 96.420 & 90 \\ -100 & 1 & 14.9727 & 14.9960 & 18.5853 & 90 & 96.382 & 90 \\ -120 & 1 & 14.9566 & 14.9709 & 18.5727 & 90 & 96.374 & 90 \\ -140 & 1 & 14.9545 & 14.9500 & 18.5611 & 90 & 96.344 & 90\end{array}$




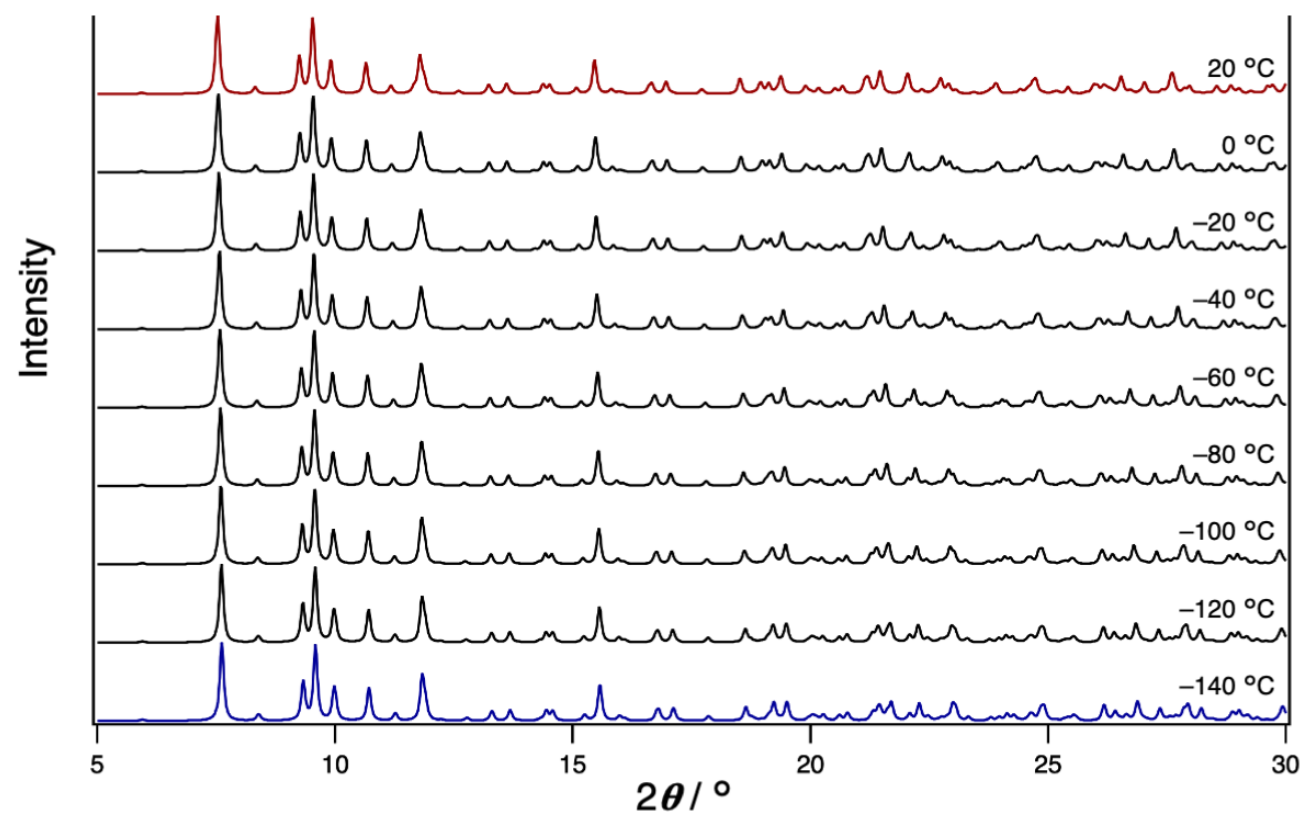

Figure S26. Simulated powder XRD patterns derived from the temperature-dependent single-crystal XRD measurements of 3 from $20^{\circ} \mathrm{C}$ to $-140{ }^{\circ} \mathrm{C}$. 


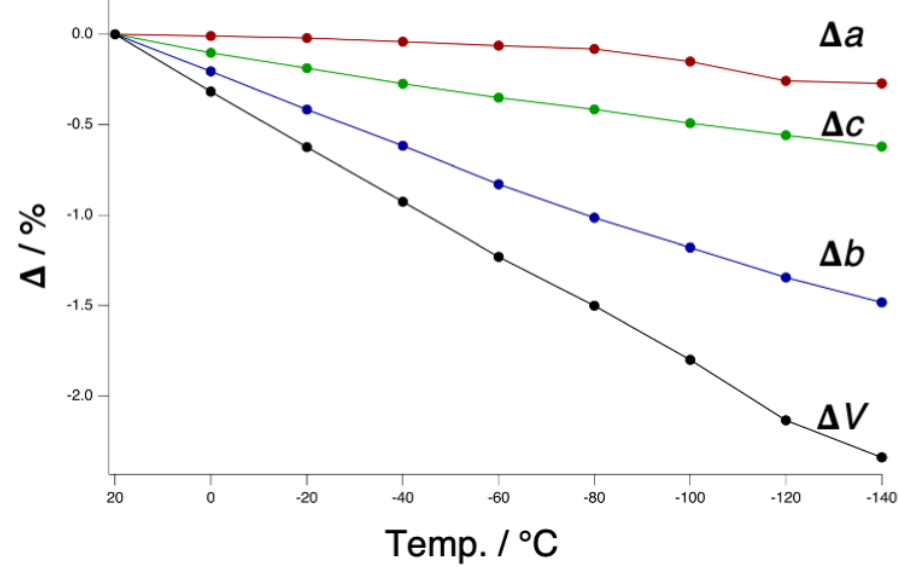

Figure S27. Plots of the changes in the crystallographic parameters $\Delta a, \Delta b, \Delta c$, and $\Delta V$ of $\mathbf{3}$ versus temperature. $\Delta a$ is defined as $\Delta a=a_{T}-a_{20}{ }^{\circ} \mathrm{C}$, in which $T$ is given temperature, $a_{T}$ and $\mathrm{a}_{20}{ }^{\circ} \mathrm{C}$ are values at temperature of $T$ and $20^{\circ} \mathrm{C}$, respectively. $\Delta b, \Delta c$, and $\Delta V$ are defined in the same manner. 


\section{NMR spectra}

${ }^{1} \mathrm{H}$ NMR spectrum of 9-isocyanideanthracene (400 $\mathrm{MHz}, \mathrm{CDCl}_{3}$ )

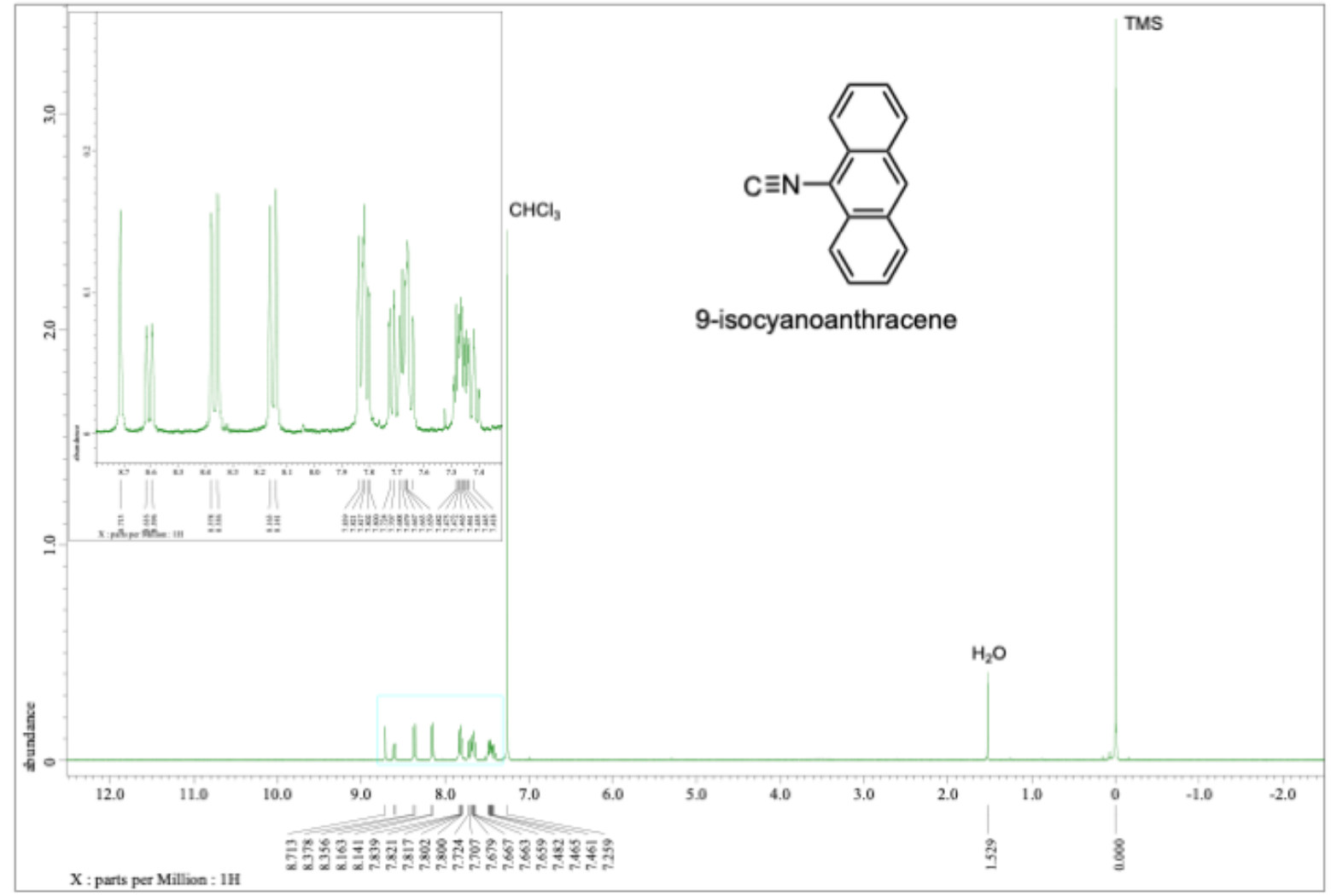

${ }^{13} \mathrm{C} \mathrm{NMR}$ spectrum of 9-isocyanideanthracene $\left(100 \mathrm{MHz}, \mathrm{CDCl}_{3}\right)$

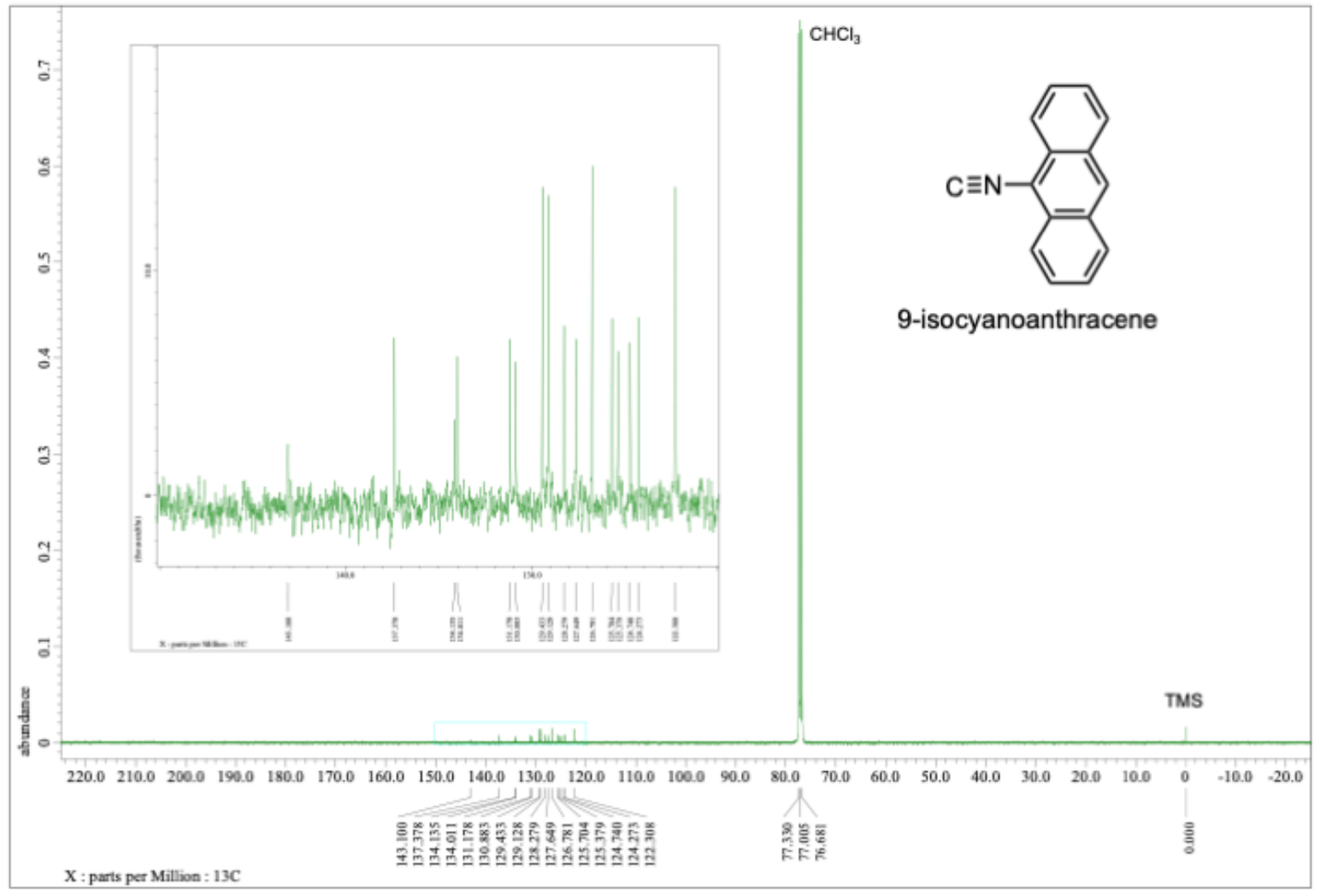


${ }^{1} \mathrm{H}$ NMR spectrum of $\mathbf{S 2}\left(400 \mathrm{MHz}, \mathrm{CDCl}_{3}\right)$

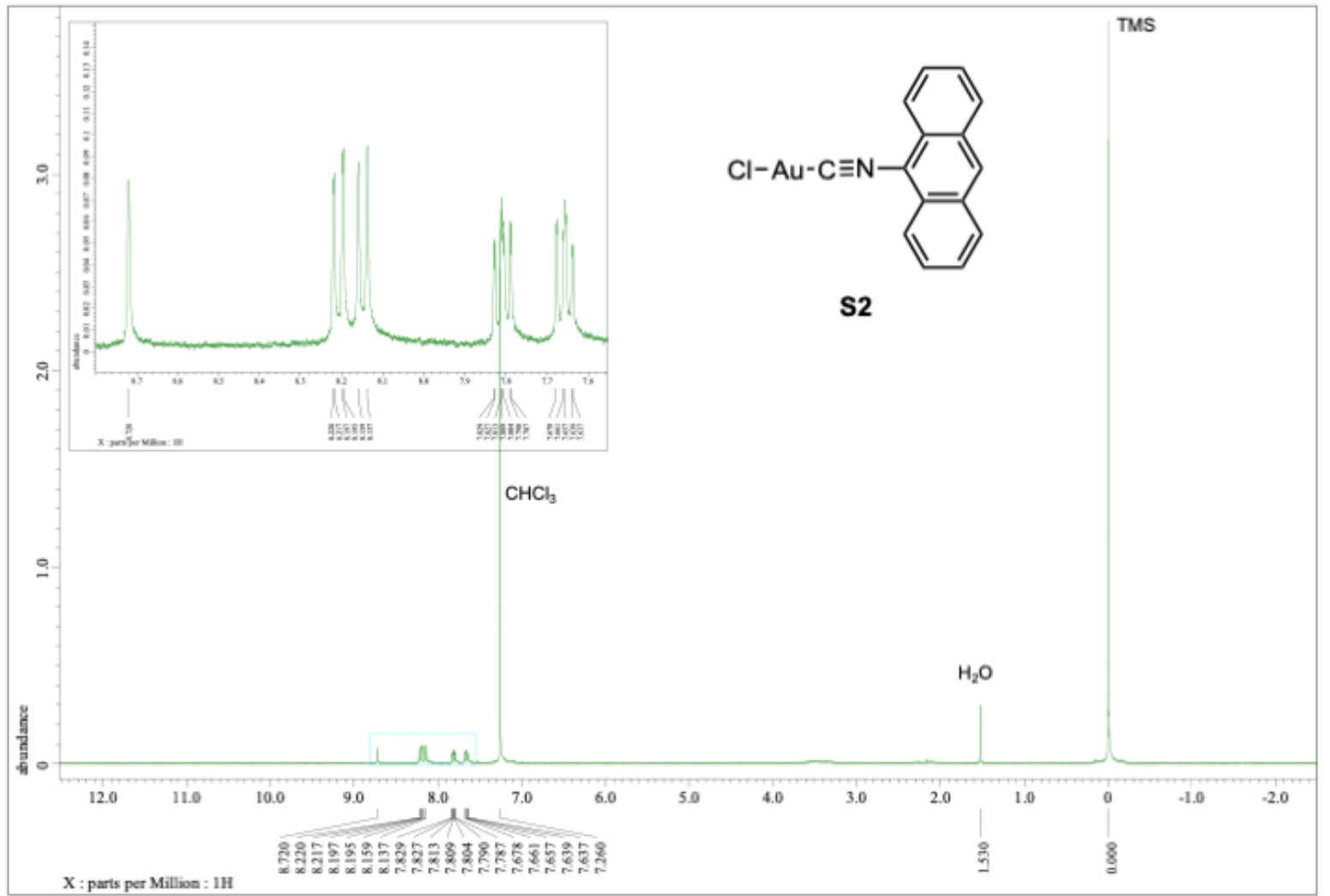

${ }^{1} \mathrm{H}$ NMR spectrum of $1\left(400 \mathrm{MHz}, \mathrm{CDCl}_{3}\right)$

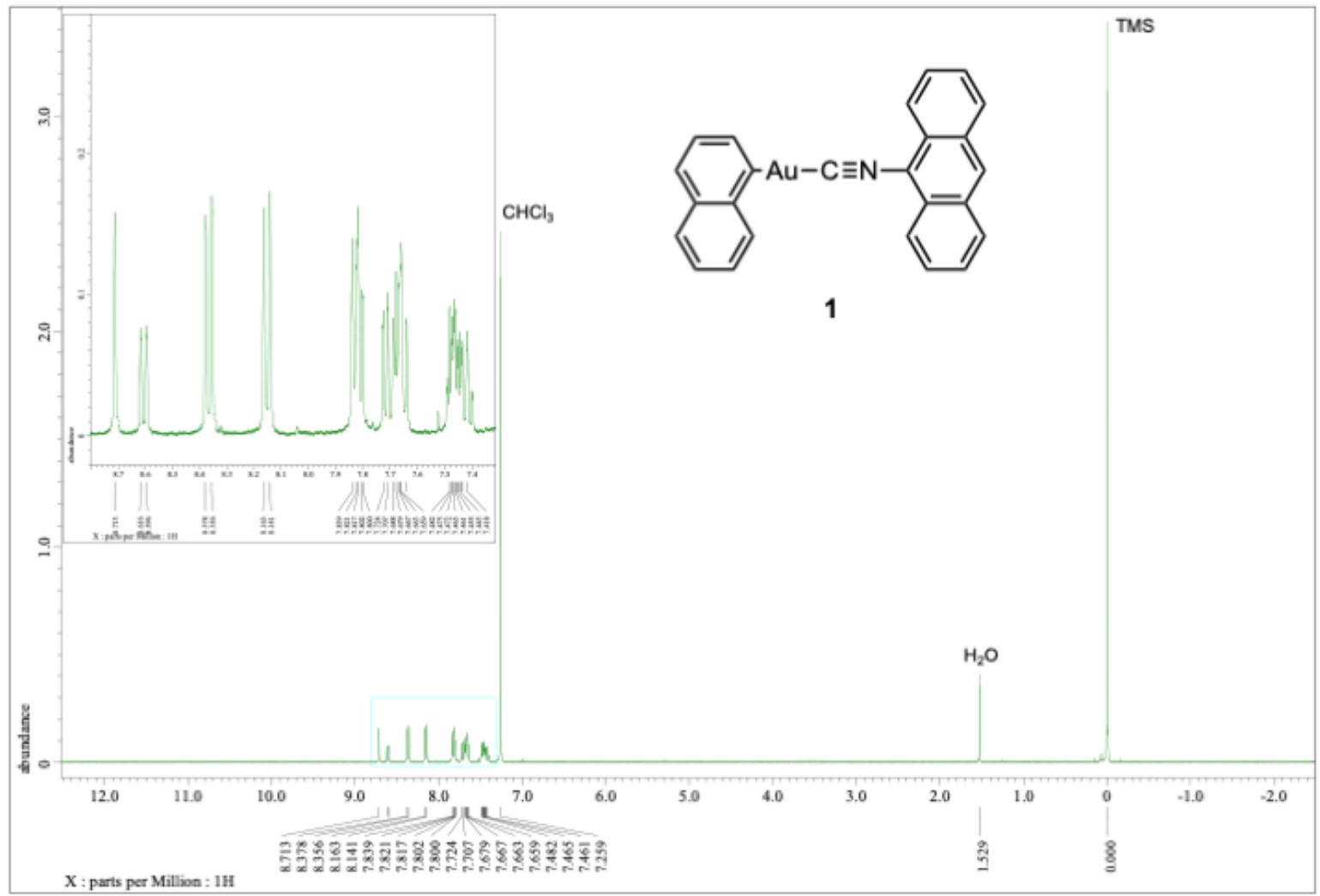


${ }^{1} \mathrm{H}$ NMR spectrum of $2\left(400 \mathrm{MHz}, \mathrm{CDCl}_{3}\right)$

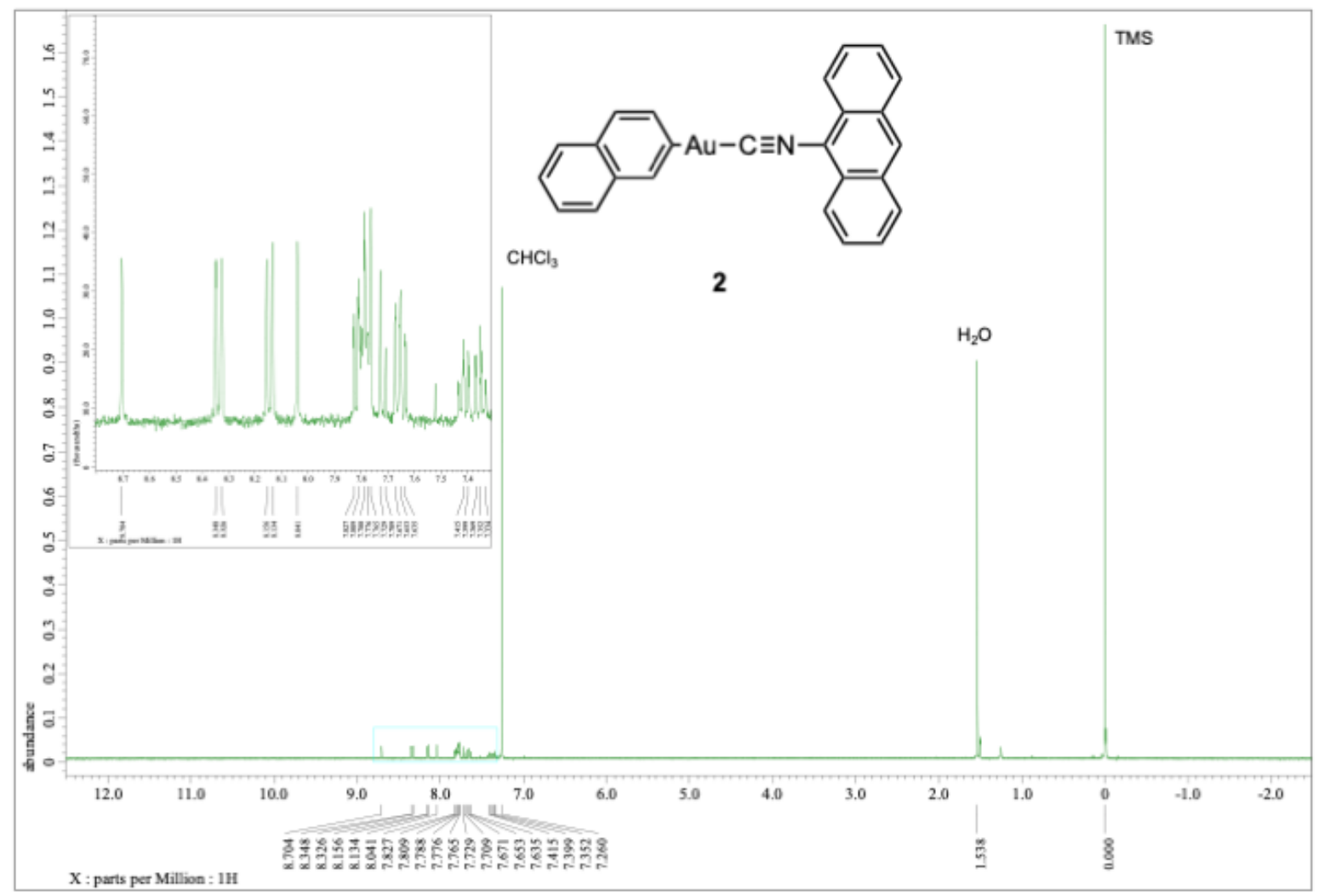

${ }^{1} \mathrm{H}$ NMR spectrum of $\mathbf{3}\left(400 \mathrm{MHz}, \mathrm{CDCl}_{3}\right)$

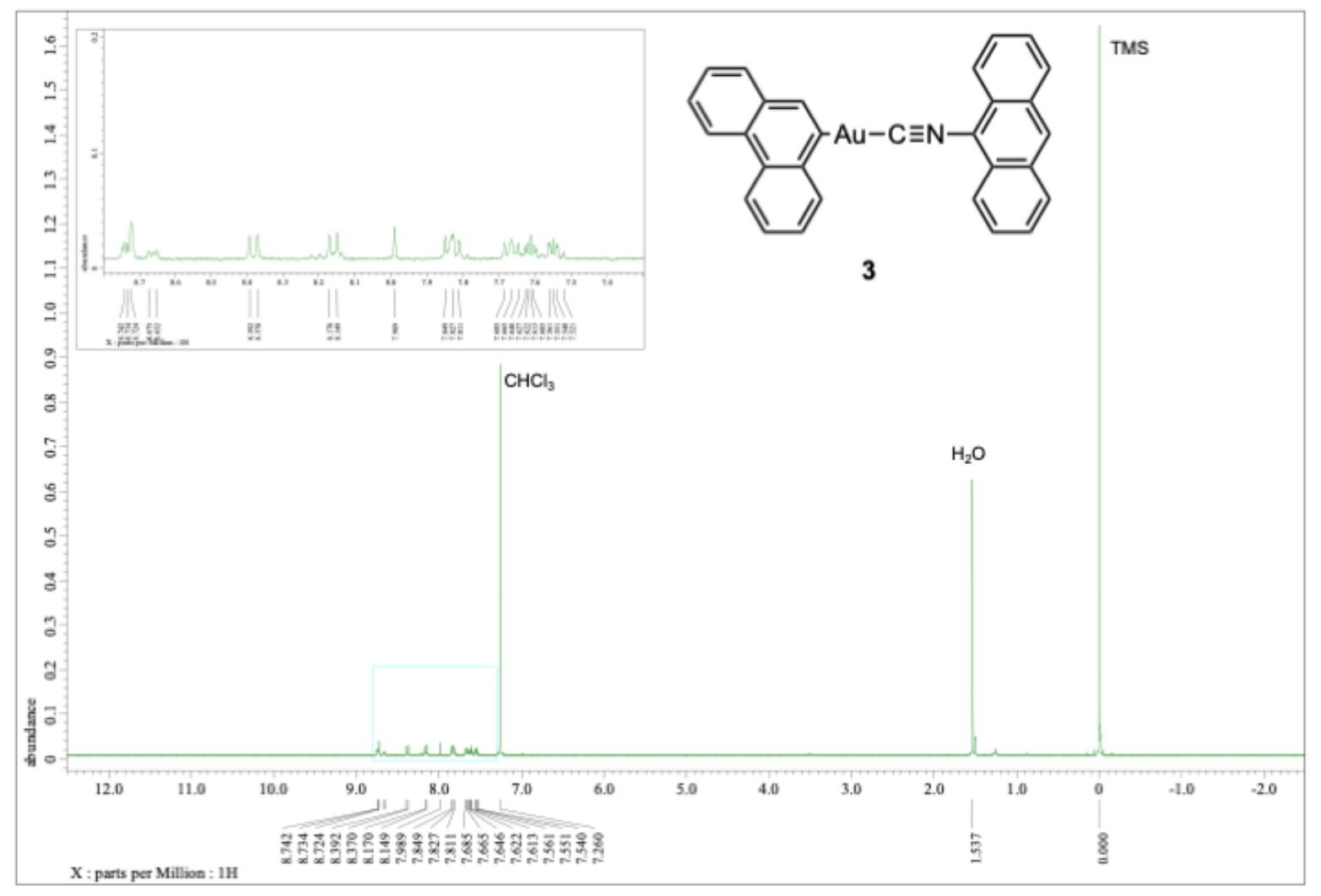




\section{Reference}

[S1] Synthesis of Chloro(tetrahydrothiophene)gold(I): Ahrland, S.; Dreisch, K.; Norén, B.; Oskarsson, Å. Metal-metal interactions in chain compounds of gold(I): syntheses and crystal structures of chlorotetrahydrothiophenegold(I), bromotetrahydrothiophenegold(I) and iodotetrahydroselenophenegold(I). Mater. Chem. Phys. 1993, 35, 281-289.

[S2] Sheldrick, G. M. SHTLXT - Integrated space-group and crystal-structure determination. Acta Cryst. 2015, A71, 3-8.

[S3] Sheldrick, G. M. Crystal structure refinement with SHELXL. Acta Cryst. 2015, C71, 3-8.

[S4] Dolomanov, O. V.; Bourhis, L. J.; Gildea, R. J.; Howard, J. A. K.; Puschmann, H. OLEX2: a complete structure solution, refinement and analysis program. J. Appl. Crystallogr. 2009, 42, 339-341. [S5] Macrae, C. F.; Sovago, I.; Cottrell, S. J.; Galek, P. T. A.; McCabe, P.; Pidcock, E.; Platings, M.; Shields, G. P.; Stevens, J. S.; Towler, M.; Wood, P. A. Mercury 4.0: from visualization to analysis, design and prediction. J. Appl. Crystallogr. 2020, 53, 226-235.

[S6] For P1, see: (a) Colombier, I.; Spagnoli, S.; Corval, A.; Baldeck, P. L.; Giraud, M.; Léaustic, A.; Yu, P. Strong photomechanical effects in photochromic organic microcrystals. Mol. Cryst. Liq. Cryst. 2005, 431, 495-499. (b) Colombier, I.; Spagnoli, S.; Corval, A.; Baldeck, P. L.; Giraud, M.; Leaustic, A.; Yu, P.; Irie, M. Diarylethene microcrystals make directional jumps upon ultraviolet irradiation. J. Chem. Phys. 2007, 126, 011101.

[S7] For P2, see: (a) Hatano, E.; Morimoto, M.; Hyodo, K.; Yasuda, N.; Yokojima, S.; Nakamura, S.; Uchida, K. Photosalient effect of a diarylethene with a perfluorocyclohexene ring. Chem. Eur. J. 2016, 22, 12680-12683. (b) Hatano, E.; Morimoto, M.; Imai, T.; Hyodo, K.; Fujimoto, A.; Nishimura, R.; Sekine, A.; Yasuda, N.; Yokojima, S.; Nakamura, S.; Uchida, K. Photosalient phenomena that mimic impatiens are observed in hollow crystals of diarylethene with a perfluorocyclohexene ring. Angew. Chem., Int. Ed. 2017, 56, 12576-2580.

[S8] For P3 and P4, see: Kitagawa, D.; Okuyama, T.; Tanaka, R.; Kobatake, S. Photoinduced rapid and explosive fragmentation of diarylethene crystals having urethane bonding. Chem. Mater. 2016, 28, 4889-4892.

[S9] For P5-7, see: Nakagawa, Y.; Morimoto, M.; Yasuda, N.; Hyodo, K.; Yokojima, S.; Nakamura, S.; Uchida, K. Photosalient effect of diarylethene crystals of thiazoyl and thienyl derivatives. Chem. Eur. J. 2019, 25, 7874-7880.

[S10] For P8, see: Naumov, P.; Kowalik, J.; Solntsev, K. M.; Baldridge, A.; Moon, J.-S.; Kranz, C.; Tolbert, L. M. Topochemistry and photomechanical effects in crystals of green fluorescent protein-like chromophores: effects of hydrogen bonding and crystal packing. J. Am. Chem. Soc. 2010, 132, 58455857.

[S11] For P9, see: Kim, T.; Zhu, L.; Mueller, L. J.; Bardeen, C. J. Dependence of the solid-state photomechanical response of 4-chlorocinnamic acid on crystal shape and size. CrystEngComm 2012, 14, 7792-7799. 
[S12] For P10 and P11, see: (a) Medishetty, R.; Husain, A.; Bai, Z.; Runčevski, T.; Dinnebier, R. E.; Naumov, P.; Vittal, J. J. Single crystals popping under UV light: a photosalient effect triggered by a [2+2] cycloaddition reaction. Angew. Chem., Int. Ed. 2014, 53, 5907-5911.

[S13] For P12, see: Yadava, K.; Vittal, J. J. Photosalient behavior of photoreactive Zn(II) complexes. Cryst. Growth Des. 2019, 19, 2542-2547.

[S14] For P13-15, see: Yadava, K.; Gallo, G.; Bette, S.; Mulijanto, C. E.; Karothu, D. P.; Park, I.H.; Medishetty, R.; Naumov, P.; Dinnebier, R. E., Vittal, J. J. Extraordinary anisotropic thermal expansion in photosalient crystals. IUCrJ 2020, 7, 83-89.

[S15] For P16, see: Medishetty, R.; Sahoo, S. C.; Mulijanto, C. E.; Naumov, P.; Vittal, J. J. Photosalient behavior of photoreactive crystals. Chem. Mater. 2015, 27, 1821-1829.

[S16] For P17, see: Nath, N. K.; Runčevski, T.; Lai, C.-Y.; Chiesa, M.; Dinnebier, R. E.; Naumov, P. Surface and bulk effects in photochemical reactions and photomechanical effects in dynamic molecular crystals. J. Am. Chem. Soc. 2015, 137, 13866-13875.

[S17] For P18, see: Mishra, M. K.; Mukherjee, A.; Ramamurty, U.; Desiraju, G. R. Crystal chemistry and photomechanical behavior of 3,4-dimethoxycinnamic acid: correlation between maximum yield in the solid-state topochemical reaction and cooperative molecular motion. IUCrJ 2015, 2, 653-660.

[S18] For P19, see: Mulijanto, C. E.; Quah, H. S.; Tan, G. K.; Donnadieu, B.; Vittal, J. J. Curved crystal morphology, photoreactivity and photosalient behaviour of mononuclear $\mathrm{Zn}$ (II) complexes. IUCrJ 2017, 4, 65-71.

[S19] For P20, see: Wang, H.; Chen, P.; Wu, Z.; Zhao, J.; Sun, J.; Lu, R. Bending, curling, rolling, and salient behavior of molecular crystals driven by [2+2] cycloaddition of a styrylbenzoxazole derivative. Angew. Chem., Int. Ed. 2017, 56, 9463-9467.

[S20] For P21, see: Mir, M. H.; Dutta, B.; Islam, S.; Khan, S. Solid-state photodimerization via [2+2] cycloaddition in metal complexes and CPs. J. Indian Chem. Soc. 2019, 96, 1297-1301.

[S21] For P22, see: Dutta, B.; Sinha, C.; Mir, M. H. The sunlight-driven photosalient effect of a 1D coordination polymer and the release of an elusive cyclobutane derivative. Chem. Commun. 2019, 55, 11049-11051.

[S22] For P23, see: Tong, F.; Xu, W.; Guo, T.; Lui, B. F.; Hayward, R. C.; Palffy-Muhoray, P.; AlKaysi, R. O.; Bardeen, C. J. Photomechanical molecular crystals and nanowire assemblies based on the [2+2] photodimerization of a phenylbutadiene derivative. J. Mater. Chem. C, 2020, 8, 5036-5044. [S23] For P24, see: Rath, B. B; Vittal, J. J. Single-Crystal-to-Single-Crystal [2 + 2] Photocycloaddition Reaction in a Photosalient One-Dimensional Coordination Polymer of $\mathrm{Pb}(\mathrm{II}) . J$. Am. Chem. Soc. 2020, 142, 20117-20123.

[S24] For P25, see: (a) Matsuura, T.; Sata, Y.; Ogura, K.; Mori, M. Photoinduced reactions. XXIII. A novel photorearrangement of santonin in the solid state. Tetrahedron Lett. 1968, 44, 4627-4630. (b) Commins, P.; Natarajan, A.; Tsai, C.-K.; Khan, S. I.; Nath, N. K.; Naumov, P.; Garcia-Garibay, M. A. Structure-reactivity correlations and mechanistic understanding of the photorearrangement and 
photosalient effect of $\alpha$-santonin and its derivatives in solutions, crystals, and nanocrystalline suspensions. Cryst. Growth Des. 2015, 15, 1983-1990.

[S25] For P26, see: (a) Naumov, P.; Sahoo, S. C.; Zakharov, B. A.; Boldyreva, E. V. Dynamic single crystals: kinematic analysis of photoinduced crystal jumping (the photosalient effect). Angew. Chem., Int. Ed. 2013, 52, 9990-9995. (b) Muya, J. T.; Meher, B. R.; Sahoo, S. C.; Chung, H. A theoretical insight into the role of counter anions and their interactions in nitropentaamminecobalt(III) toward linkage isomerism-induced photochemical motion. Int. J. Quantum Chem. 2019, 119, e25929.

[S26] For P27, see: Seki, T.; Sakurada, K.; Muromoto, M.; Ito, H. Photoinduced single-crystal-tosingle-crystal phase transition and photosalient effect of a gold(I) isocyanide complex with shortening of intermolecular aurophilic bonds. Chem. Sci. 2015, 6, 1491-1497.

[S27] For P28 and P29, see: Gupta, P.; Panda, T.; Allu, S.; Borah, S.; Baishya, A.; Gunnam, A.; Nangia, A.; Naumov, P.; Nath, N. K. Crystalline acylhydrazone photoswitches with multiple mechanical responses. Cryst. Growth Des. 2019, 19, 3039-3044.

[S28] For T1, see: (a) Etter, M. C.; Siedle, A. R. Solid-state rearrangement of (phenylazophenyl)palladium hexafluoroacetylacetonate. J. Am. Chem. Soc. 1983, 105, 641-643. (b) Etter, M. C.; Siedle, A. R. Crystal expansion during a thermochromic transformation. Mol. Cryst. Liq. Cryst. 1983, 96, 35-38. (c) Panda, M. K.; Runčevski, T.; Sahoo, S. C.; Belik, A. A.; Nath, N. K.; Dinnebier, R. E.; Naumov, P. Colossal positive and negative thermal expansion and thermosalient effect in a pentamorphic organometallic martensite. Nat. Commun. 2014, 5, 4811. (d) Khalil, A.; Karothu, D. P.; Naumov, P.; Direct quantification of rapid and efficient single-stroke actuation by a martensitic transition in a thermosalient crystal. J. Am. Chem. Soc. 2019, 141, 3371-3375.

[S29] For T2, see: (a) Gigg, J.; Gigg, R.; Payne, S.; Conant, R. The allyl group for protection in carbohydrate chemistry. Part 21. ( \pm )-1,2:5.6- and ( \pm )-1,2:3,4-di- $O$-isopropylidene-myo-inositol. The unusual behaviour of crystals of ( \pm )-3,4-di- $O$-acetyl-1,2,5,6-tetra- $O$-benzyl-myo-inositol on heating and cooling: a 'thermosalient solid'. J. Chem. Soc., Perkin Trans. 1, 1987, 2411-2414. (b) Steiner, T.; Hinrichs, W.; Saenger, W.; Gigg, R. 'Jumping crystals': X-ray structures of the three crystalline phases of ( \pm )-3,4-di-O-acetyl-1,2,5,6-tetra-O-benzyl-myo-inositol. Acta Cryst. 1993, B49, 708-718.

[S30] For T3, see: (a) Kohne, B.; Praefcke, K.; Mann, G. Perhydropyren, ein beim phasenübergang hüpfender Kohlenwasserstoff. Chimia 1988, 42, 139-141. (b) Ding, J.; Herbst, R.; Praefcke, K.; Kohne, B.; Saenger, W. A crystal that hops in phase transition, the structure of trans,trans,anti,trans,transperhydropyrene. Acta Cryst. 1991, B47, 739-742.

[S31] For T4, see: (a) Davey, R. J.; Maginn, S. J.; Andrews, S. J.; Black, S. N.; Buckley, A. M.; Cottier, D.; Dempsey, P.; Plowman, R.; Rout, J. E.; Stanley, D. R.; Taylor, A. Morphology and polymorphism of terephthalic acid. Mol. Cryst. Liq. Cryst. 1994, 242, 79-90. (b) Sahoo, S. C.; Nath, N. K.; Zhang, L.; Semreen, M. H.; Al-Tel, T. H.; Naumov, P. Actuation based on thermo/photosalient effect: a biogenic smart hybrid driven by light and heat. RSC $A d v$. 2014, 4, 7640-7647. (c) Karothu, D. P.; Weston, J.; Desta, I. T.; Naumov, P. Shape-memory and self-healing effects in mechanosalient molecular crystals. J. Am. Chem. Soc. 2016, 138, 13298-13306. 
[S32] For T5, see: (a) Zamir, S.; Bernstein, J.; Greenwood, D. J. A single crystal to single crystal reversible phase transition which exhibits the "hopping effect". Mol. Cryst. Liq. Cryst. 1994, 242, 193200. (b) Skoko, Ž.; Zamir, S.; Naumov, P.; Bernstein, J. The thermosalient phenomenon. "jumping crystals" and crystal chemistry of the anticholinergic agent oxitropium bromide. J. Am. Chem. Soc. 2010, 132, 14191-14202.

[S33] For T6, see: Corbett, J. M.; Dickman, M. H. 4,5-Bis(fluorodinitromethyl)-2-methoxy-1,3dioxolane. Acta Cryst. 1996, C52, 1851-1853.

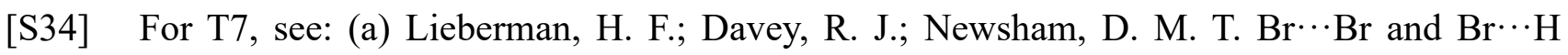
interactions in action: polymorphism, hopping, and twinning in 1,2,4,5-tetrabromobenzene. Chem. Mater. 2000, 12, 490-494. (b) Sahoo, S. C.; Sinha, S. B.; Kiran, M. S. R. N.; Ramamurty, U.; Dericioglu, A. F.; Reddy, C. M.; Naumov, P. Kinematic and mechanical profile of the self-actuation of thermosalient crystal twins of 1,2,4,5-tetrabromobenzene: a molecular crystalline analogue of a bimetallic strip. J. Am. Chem. Soc. 2013, 135, 13843-13850. (c) Khalil, A.; Ahmed, E.; Naumov, P. Metal-coated thermosalient crystals as electrical fuses. Chem. Commun. 2017, 53, 8470-8473. (d) Khalil, A.; Hu, C. T.; Naumov, P. Nanoscale crystallization and thermal behaviour of 1,2,4,5tetrabromobenzene. CrystEngComm 2018, 20, 636-642. (e) Zakharov, B. A.; Michalchuk, A. A. L.; Morrison, C. A.; Boldyreva, E. V. Anisotropic lattice softening near the structural phase transition in the thermosalient crystal 1,2,4,5-tetrabromobenzene. Phys. Chem. Chem. Phys. 2018, 20, 8523-8532. (f) Zaczek, A. J.; Catalano, L.; Naumov, P.; Korter, T. M. Mapping the polymorphic transformation gateway vibration in crystalline 1,2,4,5-tetrabromobenzene. Chem. Sci. 2019, 10, 1332-1341.

[S35] For T8, see: Yasutake, M.; Sakamoto, Y.; Onaka, S.; Sako, K.; Tatemitsu, H.; Shinmyozu, T. Crystal structural properties of a pinwheel compound: [36](1,2,3,4,5,6)cyclophane. Tetrahedron Lett. 2000, 41, 7933-7938.

[S36] For T9, see: Siegrist, T.; Besnard, C.; Haas, S.; Schiltz, M.; Pattison, P.; Chernyshov, D.; Batlogg, B.; Kloc, C. A polymorph lost and found: the high-temperature crystal structure of pentacene. Adv. Mater. 2007, 19, 2079-2082.

[S37] For T10, see: (a) Wu, H.; Reeves-McLaren, N.; Pokorny, J.; Yarwood, J.; West, A. R. Polymorphism, phase transitions, and thermal stability of L-pyroglutamic acid. Cryst. Growth Des. 2010, 10, 3141-3148. (b) Panda, M. K.; Runčevski, T.; Husain, A.; Dinnebier, R. E.; Naumov, P. Perpetually self-propelling chiral single crystals. J. Am. Chem. Soc. 2015, 137, 1895-1902. (c) Lončarić, I.; Popović, J.; Despoja, V.; Burazer, S.; Grgičević, I.; Popović, D.; Skoko, Ž. Reversible thermosalient effect of $N^{\prime}$-2-propylidene-4-hydroxybenzohydrazide accompanied by an immense negative compressibility: structural and theoretical arguments aiming toward the elucidation of jumping phenomenon. Cryst. Growth Des. 2017, 17, 4445-4453.

[S38] For T11, see: Centore, R.; Jazbinsek, M.; Tuzi, A.; Roviello, A.; Capobianco, A.; Peluso, A. A series of compounds forming polar crystals and showing single-crystal-to-single-crystal transitions between polar phases. CrystEngComm 2012, 14, 2645-2653. 
[S39] For T12 and T13, see: Panda, M. K.; Centore, R.; Causà, M.; Tuzi, A.; Borbone. F.; Naumov, P. Strong and anomalous thermal expansion precedes the thermosalient effect in dynamic molecular crystals. Sci. Rep. 2016, 6, 29610.

[S40] For T14, see: Lusi, M.; Bernstein, J. On the propulsion mechanism of "jumping" crystals. Chem. Commun. 2013, 49, 9293-9295.

[S41] For T15, see: Nauha, E.; Naumov, P.; Lusi, M. Fine-tuning of a thermosalient phase transition by solid solutions. CrystEngComm 2016, 18, 4699-4703.

[S42] For T16, see: Ghosh, S.; Mishra, M. K.; Ganguly, S.; Desiraju, G. R. Dual stress and thermally driven mechanical properties of the same organic crystal: 2,6-dichlorobenzylidene-4-fluoro-3nitroaniline. J. Am. Chem. Soc. 2015, 137, 9912-9921.

[S43] For T17, see: Engel, E. R.; Smith, V. J.; Bezuidenhout, C. X.; Barbour, L. J. Thermoresponsive organic inclusion compounds: modification of thermal expansion behavior by simple guest replacement. Chem. Mater. 2016, 28, 5073-5079.

[S44] For T18, see: Vicente, A. I.; Joseph, A.; Ferreira, L. P.; Carvalho, M. D.; Rodrigues, V. H. N.; Duttine, M.; Diogo, H. P.; Minas da Piedade, M. E.; Calhorda, M. J.; Martinho, P. N. Dynamic spin interchange in a tridentate Fe(III) Schiff-base compound. Chem. Sci. 2016, 7, 4251-4258.

[S45] For T19, see: Martins, F. F.; Joseph, A.; Diogo, H. P.; Minas da Piedade, M. E.; Ferreira, L. P.; Carvalho, M. D.; Barroso, S.; Romão, M. J.; Calhorda, M. J.; Martinho, P. N. Irreversible magnetic behaviour caused by the thermosalient phenomenon in an iron(III) spin crossover complex. Eur. $J$. Inorg. Chem. 2018, 25, 2976-2983.

[S46] For T20-23, see: Takeda, T.; Akutagawa, T. Anisotropic dissociation of $\pi-\pi$ stacking and flipping-motion-induced crystal jumping in alkylacridones and their dicyanomethylene derivatives. Chem. Eur. J. 2016, 22, 7763-7770.

[S47] For T24, see: (a) Mittapalli, S.; Perumalla, D. S.; Nangia, A. Mechanochemical synthesis of $\mathrm{N}$-salicylideneaniline: thermosalient effect of polymorphic crystals. IUCrJ 2017, 4, 243-250. (b) Werny, M. J.; Vittal, J. J. Regulating thermosalient behaviour in three polymorphs. IUCrJ 2017, 4, 202-203.

[S48] For T25, see: Shibuya, Y.; Itoh, Y.; Aida, T. Jumping crystals of pyrene tweezers: crystal-tocrystal transition involving $\pi / \pi$-to-CH/ $\pi$ assembly mode switching. Chem. Asian J. 2017, 12, 811-815. [S49] For T26, see: Ohtani, S.; Gon, M.; Tanaka, K.; Chujo, Y. A flexible, fused, azomethine-boron complex: thermochromic luminescence and thermosalient behavior in structural transitions between crystalline polymorphs. Chem. Eur. J. 2017, 23, 11827-11833.

[S50] For T27-29, see: Mittapalli, S.; Perumalla, D. S.; Nanuboluc, J. B.; Nangia, A. Thermomechanical effect in molecular crystals: the role of halogen-bonding interactions. IUCrJ 2017, 4, 812-823.

[S51] For T30, see: Tamboli, M. I.; Karothu, D. P.; Shashidhar, M. S.; Gonnade, R. G.; Naumov, P. Effect of crystal packing on the thermosalient effect of the pincer-type diester naphthalene-2,3-diylbis(4-fluorobenzoate): a new class II thermosalient solid. Chem. Eur. J. 2018, 24, 4133-4139. 
[S52] For T31 and T32, see: Rawat, H.; Samanta, R.; Bhattacharya, B.; Deolka, S.; Dutta, A.; Dey, S.; Raju, K. B.; Reddy, C. M. Thermosalient forms: carryover of thermosalient behavior of coformers from single component to multicomponent forms? Cryst. Growth Des. 2018, 18, 2918-2923.

[S53] For T33, see: Raju, K. B.; Ranjan, S.; Vishnu, V. S.; Bhattacharya, M.; Bhattacharya, B.; Mukhopadhyay, A. K.; Reddy, C. M. Rationalizing distinct mechanical properties of three polymorphs of a drug adduct by nanoindentation and energy frameworks analysis: role of slip layer topology and weak interactions. Cryst. Growth Des. 2018, 18, 3927-3937.

[S54] For T34, see: Singh, M.; Chopra, D. Diversity in mechanical response in donor-acceptor coupled cocrystal stoichiomorphs based on pyrene and 1,8-dinitroanthraquinone systems. Cryst. Growth Des. 2018, 18, 6670-6680.

[S55] For T35, see: Alimi, L. O.; van Heerden, D. P.; Lama, P.; Smith, V. J.; Barbour, L. J. Reversible thermosalience of 4-aminobenzonitrile. Chem. Commun. 2018, 54, 6208-6211.

[S56] For T36, see: Singh, M.; Bhandary, S.; Bhowal, R.; Chopra, D. Observation of bending, cracking and jumping phenomena on cooling and heating of tetrahydrate berberine chloride crystals. CrystEngComm 2018, 20, 2253-2257.

[S57] For T37, see: Dharmarwardana, M.; Arimilli, B. S.; Luzuriaga, M. A.; Kwon, S.; Lee, H.; Appuhamillage, G. A.; McCandless, G. T.; Smaldone, R. A.; Gassensmith, J. J. The thermo-responsive behavior in molecular crystals of naphthalene diimides and their 3D printed thermochromic composites. CrystEngComm 2018, 20, 6054-6060.

[S58] For T38, see: So, H.-S.; Minami, T.; Jindo, T.; Matsumoto, S. Thermosalient effect of two polymorphs of a diketopyrrolopyrrole dye with different crystal systems and molecular arrangements. CrystEngComm 2018, 20, 5317-5320.

[S59] For T39, see: Colin-Molina, A.; Karothu, D. P.; Jellen, M. J.; Toscano, R. A.; Garcia-Garibay, M. A.; Naumov, P.; Rodríguez-Molina, B. Thermosalient amphidynamic molecular machines: Motion at the molecular and macroscopic scales. Matter. 2019, 1, 1033-1046.

[S60] For T40, see: Takeda, T.; Ozawa, M.; Akutagawa, T. Jumping crystal of a hydrogen-bonded organic framework induced by the collective molecular motion of a twisted $\pi$ system. Angew. Chem., Int. Ed. 2019, 58, 10345-10352.

[S61] For T41, see: Seki, T.; Mashimo, T.; Ito, H. Anisotropic strain release in a thermosalient crystal: correlation between the microscopic orientation of molecular rearrangements and the macroscopic mechanical motion. Chem. Sci. 2019, 10, 4185-4191.

[S62] For T42, see: Gaztañaga, P.; Baggio, R.; Halac, E.; Vega, D. R. Thermal, spectroscopic and structural analysis of a thermosalient phase transformation in tapentadol hydrochloride. Acta Cryst. 2019, B75, 183-191.

[S63] For T43, see: Jin, J.; Wu, S.; Ma, Y.; Dong, C.; Wang, W.; Liu, X.; Xu, H.; Long, G.; Zhang, M.; Zhang, J.; Huang, W. Nucleation control-triggering cocrystal polymorphism of charge-transfer complexes differing in physical and electronic properties. ACS Appl. Mater. Interfaces 2020, 12, 19718-19726. 
[S64] For T44, see: Omoto, K.; Nakae, T.; Nishio, M.; Yamanoi, Y.; Kasai, H.; Nishibori, E.; Mashimo, T.; Seki, T.; Ito, H.; Nakamura, K.; Kobayashi, N.; Nakayama, N.; Goto, H.; Nishihara, H. Thermosalience in macrocycle-based soft crystals via anisotropic deformation of disilanyl architecture. J. Am. Chem. Soc. 2020, 142, 12651-12657.

[S65] For T45, see: Klaser, T.; Popović, J.; Fernandes, J. A.; Tarantino, C. S.; Zema, M.; Skoko, Ž. Does thermosalient effect have to concur with a polymorphic phase transition? The case of methscopolamine bromide. Crystals 2018, 8, 301.

[S66] For T46, see: Smets, M. M. H.; Kalkman, E.; Krieger, A.; Tinnemans, P.; Meekes, H.; Vlieg, E.; Cuppen, H. M. On the mechanism of solid-state phase transitions in molecular crystals - the role of cooperative motion in (quasi)racemic linear amino acids. IUCrJ 2020, 7, 331-341.

[S67] For T47 and T48, see: Jin, M.; Yamamoto, S.; Seki, T.; Ito, H.; Garcia-Garibay, M. A. Anisotropic thermal expansion as the source of macroscopic and molecular scale motion in phosphorescent amphidynamic crystals. Angew. Chem., Int. Ed. 2019, 58, 18003-18010.

[S68] For T49-51, see: Seki, T.; Mashimo, T.; Ito, H. Crystal jumping of simple hydrocarbons: cooling-induced salient effect of bis-, tri-, and tetraphenylethene through anisotropic lattice dimension changes without thermal phase transitions. Chem. Lett. 2020, 49, 174-177.

[S69] PASCal: Cliffe, M. J.; Goodwin, A. L. PASCal: a principal axis strain calculator for thermal expansion and compressibility determination. J. Appl. Cryst. 2012, 45, 1321-1329. 